Feeding Interactions and Their Relevance to Biodiversity under Global Change

Yuanheng Li 



\title{
Feeding Interactions and Their Relevance to Biodiversity under Global Change
}

\author{
Dissertation \\ for the award of the degree \\ "Doctor of Philosophy" Ph.D. Division of Mathematics and Natural Sciences \\ in the Faculty of Biology and Psychology \\ of the Georg-August-Universität Göttingen \\ within the doctoral program Biology \\ of the Georg-August University School of Science (GAUSS)
}

submitted by

Yuanheng Li

from Shijiazhuang, China

Göttingen

Mar. 03, 2017 
Thesis Committee:

Members of the Examination Board:

Reviewer:

Second Reviewer:

Further Members

of the Examination Board:
Prof. Dr. Ulrich Brose,

Friedrich Schiller University Jena

Prof. Dr. Kerstin Wiegand,

Georg-August-Universität Göttingen

Prof. Dr. Ulrich Brose,

Friedrich Schiller University Jena

Prof. Dr. Kerstin Wiegand,

Georg-August-Universität Göttingen

Prof. Dr. Stefan Scheu,

Georg-August-Universität Göttingen

Prof. Dr. Holger Kreft,

Georg-August-Universität Göttingen

Dr. Matthias Waltert,

Georg-August-Universität Göttingen

Dr. Oliver Schülke,

Georg-August-Universität Göttingen

Date of the oral examination: Mar. 17, 2017 



\section{Summary}

Feeding interaction strengths are key in characterizing ecological networks. They are of great importance to the dynamics and stability of the networks (hereby food webs) and biodiversity. Even though feeding interactions have been studied for more than one century, there still exist many aspects that need to be addressed in order to better bring studies of feeding interactions and food webs together. Feeding interactions are normally investigated in short-term and at individual-level, whereas, food-web studies are normally explored from the perspectives of long-term, population- and community-level. Especially, how to bring them together under current global change is even less studied. Therefore, I focused on two processes occurred in feeding, experimental duration and satiation level of predators in the first research chapter. I used a meta-analytical approach to investigate their effects on estimating feeding interaction strengths (i.e. functional response parameters). I gained not only a better mechanistic understanding towards feeding interactions, but also how to better quantify interaction strengths in the population-and community-level studies. In lights of the first study, I generated an individual-based model (IBM) to mimic the predator-prey feeding experiments in the laboratory and investigated the influence of one global change driver, habitat degradation (represented by habitat loss and habitat simplification). This IBM model allowed me to systematically investigate the effects of habitat loss and habitat simplification on the estimates of interaction strengths, which is operationally not possible for empirical studies. Moreover, in this second study, I combined the individual-level IBM with a population-level model of ordinary differential equations (ODE) to upscale the effects of habitat degradation to a predator-prey system. Finally, in the last research chapter of this thesis, I analyzed an extensive set of empirical functional responses to quantify the combined effects of two global change drivers, habitat loss and global warming, on the estimates of interaction strengths. Following the new scalings of functional response parameters from this analysis, I illustrated the synergistic effects of habitat loss and global warming on biodiversity by an ODE model of a food chain (a basic food-web motif).

The result of my first study highlighted the importance of experimental duration and predator satiation on functional response parameters. The attack rate decreased with increasing experimental duration and the handling times for starved predators 
were lower than those for the satiated predators. These results also pointed out the importance of digestive processes for feeding interactions and estimates of functional response parameters. Thereafter, I generated an IBM model of feeding interactions with empirically supported and digestively elaborated physiological processes. The results of the IBM model showed that feeding efficiency (i.e. halfsaturation density) decreased with increasing habitat complexity (presented by prey refuge availability) but was not influenced by patch size (marginally increased with patch size). The results from the analytical ODE model illustrated that patch size needs to be larger that a baseline to be able to support both predator and prey populations and increasing habitat complexity can decrease such baseline. The third study illustrated, for the first time, that the feeding efficiency increased with patch size and confirmed that the maximum feeding rate increased with temperature. Combined with other important rates of energy flux which scale with body mass and temperature by empirically-supported values, the food chain models suggested that habitat loss fosters the negative effect of warming on biodiversity, leading to higher extinction risks of consumers at higher trophic levels.

This thesis contributes to a better understanding of feeding interactions and presents new theoretical tools to predict the response of ecological systems to global change factors. For future studies, the importance of ,experimental duration' and ,satiation level of predator' could be proven helpful when designing new studies of feeding interactions and quantifying feeding interaction strengths in populationor community-level studies. It would also be important to further investigate the physiological mechanisms of how patch size affects the feeding efficiency. For global change research, my studies also highlight the importance to investigate multiple drivers of global environmental change (GEC) and emphasis that quantifying the effects of GECs on the individual biological rates and combining these rates through a network structure allow us to better explore their effects on biodiversity. 


\title{
Author contributions
}

\author{
Chapter 2 \\ Effects of experimental duration and satiation level of predator on the deriva- \\ tion of functional response parameters \\ Yuanheng Li, Björn C. Rall, Gregor Kalinkat
}

G.K. and B.C.R. designed the study; Y.L. analyzed the meta dataset in the light of discussion with B.C.R. and G.K.; Y.L. wrote the manuscript with substantial further contributions from two other authors.

\section{Chapter 3}

How patch size and refuge availability change interaction strength and population dynamics: a combined individual- and population-based modeling experiment

Yuanheng Li, Ulrich Brose, Katrin Meyer, Björn C. Rall

B.C.R. and Y.L. designed the study; Y.L. constructed the simulation model; Y.L. and B.C.R. analyzed the simulation data; Y.L. and B.C.R. constructed and analyzed the differential equation model; Y.L. and B.C.R. wrote the manuscript with contributions from two other authors.

Published in PeerJ, 2017, 5:e2993, doi: 10.7717/peerj.2993

(C) 2017 Li et al.

\section{Chapter 4}

Joint effects of habitat loss and warming alter species interactions and enhance biodiversity loss

Yuanheng Li, Gregor Kalinkat, Björn C. Rall

Y.L., G.K. and B.C.R. designed the study; Y.L. analyzed the meta dataset in the light of discussion with B.C.R. and G.K.; Y.L. and B.C.R. constructed the simulation model; Y.L. wrote the manuscript with substantial further contributions from two other authors. 



\title{
Affiliations of co-authors
}

\author{
Ulrich Brose \\ German Centre for Integrative Biodiversity Research (iDiv), \\ Halle-Jena-Leipzig \\ Deutscher Platz 5e, 04103 Leipzig, Germany \\ Institute of Ecology \\ Friedrich Schiller University Jena, Dornburger-Str. 159 \\ 07743 Jena, Germany \\ Gregor Kalinkat \\ Department of Biology and Ecology of Fishes \\ Leibniz-Institute of Freshwater Ecology and Inland Fisheries \\ Müggelseedamm 310, 12587 Berlin, Germany \\ Katrin Meyer \\ Department of Ecosystem Modelling \\ Georg-August-Universität Göttingen \\ Büsgenweg 4, 37077 Göttingen, Germany \\ Björn C. Rall \\ German Centre for Integrative Biodiversity Research (iDiv), \\ Halle-Jena-Leipzig \\ Deutscher Platz 5e, 04103 Leipzig, Germany \\ Institute of Ecology \\ Friedrich Schiller University Jena, Dornburger-Str. 159 \\ 07743 Jena, Germany
}





\section{Contents}

Summary vii

$\begin{array}{ll}\text { Author contributions } & \text { ix }\end{array}$

Affiliations of co-authors $\quad x i$

I General introduction 1

1 Introduction $\quad 3$

Feeding interaction and functional response model . . . . . . . . . . . 4

From functional response to biodiversity . . . . . . . . . . . . . 7

How global change comes into play . . . . . . . . . . . . . . . . . . . . . . . .

Outline of research chapters . . . . . . . . . . . . . . . 10

II Research chapters 13

2 Experimental duration and predator satiation level affect functional response parameters $\quad 15$

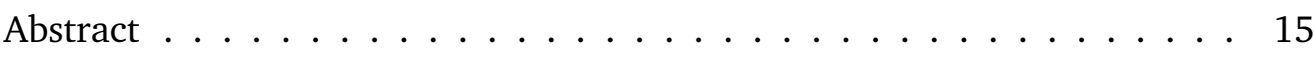

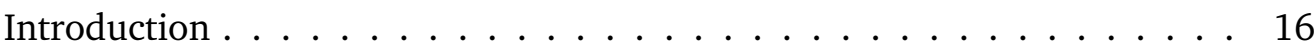

Methods . . . . . . . . . . . . . . . . . . . . . . . 19

Results .......................... 21

Discussion ....................... 22

3 How patch size and refuge availability change interaction strength and population dynamics: a combined individual- and population-based $\begin{array}{lr}\text { modeling experiment } & 27\end{array}$

Abstract . . . . . . . . . . . . . . . . . . . . 27

Introduction . . . . . . . . . . . . . . . . . . . . 29

Methods ........................... . . 31

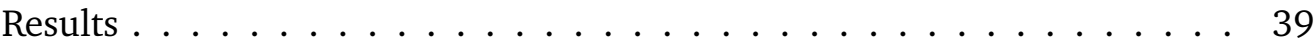

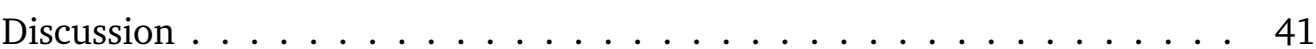

Conclusions . . . . . . . . . . . . . . . . . . . 44

Acknowledgements . . . . . . . . . . . . . . . . . . . 45 
4 Joint effects of habitat loss and warming alter species interactions and enhance biodiversity loss

Abstract . . . . . . . . . . . . . . . . . . . 4 47

Introduction . . . . . . . . . . . . . . . . . . 48

Methods . . . . . . . . . . . . . . . . . . 52

Results . . . . . . . . . . . . . . . . . 55

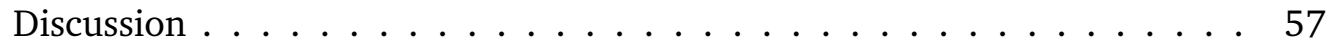

III General discussion 63

5 Discussion $\quad 65$

$\begin{array}{ll}\text { IV Appendix } & 71\end{array}$

$\begin{array}{ll}\text { Bibliography } & 73\end{array}$

$\begin{array}{lr}\text { Supporting information for Chapter } 2 & 87\end{array}$

$\begin{array}{lr}\text { Supporting information for Chapter } 3 & 89\end{array}$

$\begin{array}{lr}\text { Supporting information for Chapter } 4 & 99\end{array}$

$\begin{array}{lr}\text { Acknowledgement } & 105\end{array}$

$\begin{array}{ll}\text { Declaration } & 107\end{array}$

$\begin{array}{lr}\text { Curriculum Vitae } & 109\end{array}$ 
Part I

General introduction 



\section{Introduction}

Physics is mathematics not because we know too much about the physical world, but because we know too little; the only thing we can discover are the mathematical properties of the physical system.

- Bertrand Russell

\section{Biodiversity and species interactions under global change}

The effect of global change on biodiversity has become a major concern in ecology (Kareiva et al., 1992; Vinebrooke et al., 2004). Five major drivers of global environmental change, i.e. land-use change, climate change, nitrogen deposition, elevated atmospheric $\mathrm{CO}_{2}$ level and biotic exchange have pronounced influences on natural ecosystems (Sala et al., 2000). Atmospheric $\mathrm{CO}_{2}$ and $\mathrm{N}$ deposition have received the most attention (Tylianakis et al., 2008) and biotic exchange influences mostly freshwater ecosystems (Sala et al., 2000). Therefore, I focused on relatively less explored drives. I explored the impact of land-use change with the association of climate change in this thesis. Land-use change pervades more than three quarters of our planet (Seto et al., 2014) and its consequences such as habitat loss and habitat simplification constitute the primary driver of biodiversity loss (Brook et al., 2008). Climate change (e.g. warming) can increase extinction risk of a large fraction of species in both terrestrial and freshwater ecosystems (IPCC, 2014b). Further, more and more studies start to focus on the synergistic effects of multiple global change drivers and suggest that biodiversity is threatened most by the synergy of climate change and other environmental changes (e.g. Sala et al., 2000; Brook et al., 2008; IPCC, 2014b).

Species living in the ecosystems are not isolated from each other. They are linked together by intra- and inter-specific interactions and hence these species and their linking structure form ecological networks (Ings et al., 2009; Kéfi et al., 2012). A fundamental type of ecological networks is the food web, describing „who eats whom". Feeding interaction strength is a core aspect in characterizing the material and energy flux in food webs, as the species in such networks are predominantly linked by feeding interactions. Consumers, being at higher trophic levels, gain material and energy to support their lives via consuming their resource species. The 
material and energy flowing through such networks support the species existence and are crucial for the biodiversity in the nature.

The influences of land-use change and climate change are not only reflected in biodiversity loss, but their effects also pervade populations of species at each trophic levels; however, the magnitude and direction of their effects on different populations and trophic groups vary substantially (Tylianakis et al., 2008; Bowler et al., 2017). To disentangle the diverse responses of species to global change drivers and to gain a better understanding of the mechanisms behind the individual and synergistic effects of habitat degradation (loss and simplification) and global warming, I focus on feeding interactions and address their relevance to biodiversity from the viewpoint of energy flux in food webs in this thesis.

\section{Feeding interaction and functional response model}

\section{Feeding processes}

Feeding interactions are composed of the processes involved in consumers' activities of foraging and consumption. Due to different foraging natures of species, it can contain up to ten (or more) components, such as „rate of successful search“ (i.e. attack rate), „time exposed“ (i.e. period when predator and prey encounter), „handling time“, „hunger" and so on (Holling, 1966). The first three components mentioned (rate of successful search, time exposed and handling time) describe phenomena that occur in feeding processes. In contrast, the component, hunger' inserts its effect into the phenomenological components. For instance, a predator's hunger level would influence its willingness to forage and affect the ,rate of successful search' (Jeschke, 2007). Both these phenomenal and mechanistic components are important for the construction of in silico feeding experiments which is studied in the second research chapter (chapter 3).

\section{Functional response models}

Other than viewing feeding interactions as different processes and components, feeding interactions can also be mathematically described by functional response models which can date back to the 1940s (Solomon, 1949). Such models quantify the feeding rates as a function of resource densities. Functional response models have been widely used in population and community ecology as they connect individual feeding studies with population dynamics. Functional response models with empirically derived values allow to quantify the part of energy flowing through the specific feeding link where the values are derived from. Even though there exists many different functional response models (Jeschke et al., 2002), most of them are derived from three models, i.e. Holling's type I, type II and type III functional 
responses. However, the type I functional response is density-independent and has been suggested to be an artifact (Sarnelle and Wilson, 2008).

The type II and type III functional responses can be combined by an descendant model by Real (1977), which can be formulated as:

$$
f(N)=\frac{a N^{h}}{1+a T_{h} N^{h}} .
$$

In Eq. (1.1), the feeding rate of the consumer $f(N)$, is expressed as a function of resource density $N$, characterized by three parameters, attack rate $a$, handling time $T_{h}$ and the Hill exponent $h$. Even though the first parameter $a$, is called ,attack rate, it actually describes the space (area or volume) that a consumer successfully searches in a unit of time. The second parameter $T_{h}$, describes the time that a consumer needs to attack and subdue a resource item. When the Hill exponent equals one, the equation (Eq. (1.1)) represents the most-widely used model of functional responses, i.e. the type II functional response (Holling, 1959b). The graphic representation of the type II functional response is an asymptotic line approaching a maximum of feeding rate (Fig. 1.1A). When the Hill exponent is larger than one, the equation stands for the type III functional response, which turns to a sigmoid curve (Fig. 1.1B). Consequently, it is called the „strict“ type III functional response when the Hill exponent equals two (Holling, 1959a). Type III functional responses have important applications to food web studies and biodiversity, as they have stabilizing effects on population dynamics and therefore supporting more species (Brose et al., 2006b; Rall et al., 2008). Fig. 1.1B shows that for a functional response with the Hill exponent larger than unity, the feeding efficiencies of consumer at low resource densities are reduced. Therefore, the „top-down“ pressure (from consumer to resource) is weaken, changing dynamic populations to stable (Williams and Martinez, 2004b). Due to the importance of the Hill exponent for biodiversity, it is one of my focuses in the second research chapter (chapter 3). Yet due to the limitation of available data, I am not able to investigate this parameter in the first and third research chapter (chapter 2 and 4).

Other than the set of functional response parameters elucidated above, Real's functional response model (Eq. (1.1)) can also be described by maximum feeding rate and half saturation density:

$$
f(N)=\frac{f_{\max } N^{h}}{N_{0}{ }^{h}+N^{h}} .
$$

The maximum feeding rate $f_{\max }$, illustrates the limit of feeding rate and the half saturation density $N_{0}$, is the resource density when the feeding rate of consumer reaches half of the maximum feeding rate (Fig. 1.1B). These two sets of functional response 
$N_{0}=\frac{1}{a T_{h}}$,
parameters are not isolated with each other, $\quad f_{\max }=\frac{1}{T_{h}}$.

as they are interconvertible. When the Hill exponent turns from one to two, the half saturation density would increase and therefore a consumer would become less efficient (Fig. 1.1B). As the parameters handling time and attack rate have a more direct connection with processes and components of feeding, the researchers who conduct empirical feeding studies often use this set of functional response parameters. Whereas, maximum feeding rate and half saturation density describe the functional response model better. Therefore, I chose to use handling time and attack rate in the first research chapter (chapter 2) and maximum feeding rate and half saturation density in the last two research chapters (chapter 3 and 4) where I upscaled the effect of feeding interaction to population levels.
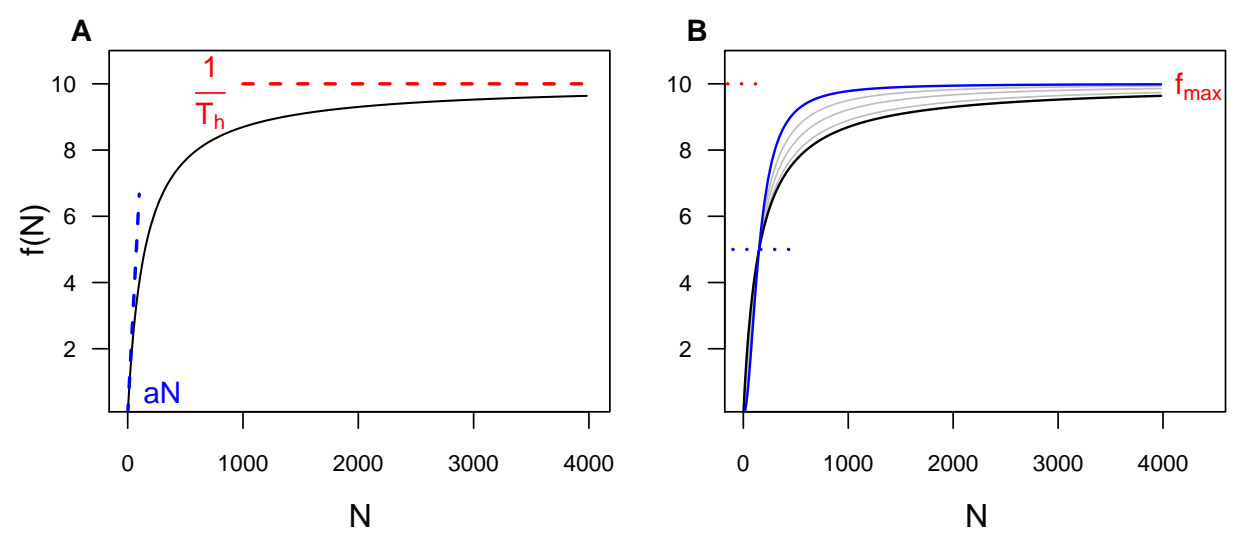

Fig. 1.1: Functional response curves with prey density as $\mathrm{x}$-axis and feeding rate as $\mathrm{y}$-axis. In panel $\mathrm{A}$, a type II functional response is present which is characterized by attack rate $(a)$ and handling time $\left(T_{h}\right)$. In panel $\mathrm{B}$, the sigmoid blue curve represents the strict type III functional response $(h=2)$ and the asymptotic black curve type II functional response $(h=1)$. The gray curves in between represent the functional responses where the Hill exponents are between 1 and 2 . All the functional responses in panel $\mathrm{B}$ have the same maximum feeding rates $\left(f_{\max }\right)$ and the blue dotted line stands for the half of $f_{\max }$.

Other than the individual-based explanation of functional response, Eq. (1.1) and (1.2) can be converted to a biomass-based function by dividing prey density $(N)$ into prey body mass and dividing feeding rate $(f(N))$ into predator-prey body-mass ratio. The biomass-based functional response, then, describes how much prey biomass is eaten by a unit of predator biomass as a function of prey biomass density. Such conversion is important for combining individual-based feeding experiments and population-level food-web studies. Food-web studies are normally modeled by ODEs and each ODE in such models stands for the biomass change of one population 
(e.g. Brose et al., 2006b). Whereas, empirical feeding studies usually quantify the feeding rates for one predator individual (e.g. Holling, 1959a). For example, in the first research chapter (chapter 2) I focused on some aspects occurring in conducting feeding experiments, therefore, individual-based functional response with attack rate and handling time was used. Whereas, in the third research chapter (chapter 4), my study objective is to illustrate the effects of global change on networklevel, thereafter, biomass-based functional response with half saturation density and maximum feeding rate was chosen. In the second research chapter (chapter 3), I chose to use the set of half saturation density and maximum feeding rate. Yet, both individual- and biomass-based functional responses were used as I quantified both in silico feeding experiments and population dynamics models.

\section{From functional response to biodiversity}

\section{Other physiological rates and population dynamics model}

To document the material and energy transfer (henceforth, energy flux) between species in an ecological network, further pathways of energy flux (other than feeding) also need to be considered. The original energy input of an ecosystem comes from autotrophic species on the lowest level of food webs since they obtain energy from abiotic sources, e.g. solar or chemical energy and fix these energies as their own biomasses (refer to intrinsic growth rate of basal species). However, the abiotic sources are not infinite: they are only capable of supplying a certain amount of autotrophic biomasses (e.g. due to micro- or macro-nutrient limitation). That is to say there is environmental carrying capacity. The intrinsic growth rate of basal species and environmental carrying capacity, together describe the energy input of basal species, logistic growth (mathematical formula below, first item of Eq. (1.4a)). To be noticed, the logistic growth integrates the respiration of basal species already. Other than being eaten by their consumers, the heterotrophic species (consumers) have another major pathway of energy loss, metabolism. The metabolic rate quantifies the basic metabolic demand of a species per unit of time (Brown et al., 2004).

To illustrate how the feeding and non-feeding energy pathways mentioned above integrally change species in ecological networks, I use ordinary differential equations (ODEs) to describe changes of population densities of species in the networks (food webs). Such mathematical abstraction comes from a bioenergetic consumer-resource model originally developed by Yodzis and Innes (1992): 


$$
\begin{aligned}
& \dot{B}_{i}=r B_{i}\left(1-\frac{B_{i}}{K}\right)-f\left(B_{i}\right) B_{j}, \\
& \dot{B}_{j}=\operatorname{ef}\left(B_{i}\right) B_{j}-x_{j} B_{j} .
\end{aligned}
$$

The resource population $i$, gains biomass by growth and loses biomass through being fed on by the consumer species $j$. The resource growth is described by the logistic growth with the intrinsic growth rate $r$, and environmental carrying capacity $K$. The consumer population loses biomass due to the metabolism $x_{c}$ and gains biomass through feeding. Not all the biomass obtained by feeding is converted into predator biomass which is determined by an assimilation efficiency $e$. The feeding interaction is characterized by consumer biomass $B_{c}$, and feeding interaction strength which is quantified by type II or type III functional responses $f\left(B_{r}\right)$. All the feeding and non-feeding physiological rates and densities that I used in this thesis are based on empirically derived paradigms and values which will be explained in the following section.

This bioenergetic model can be upgraded to a food chain. It is a linked path, excluding loops, from a consuming species to a basal (resource) species (Williams and Martinez, 2004a). Even though a food chain is the simplest food web motif of three species, I can still infer basic patterns on how global change affects biodiversity through them (Williams and Martinez, 2004a; Binzer et al., 2012). Nevertheless, combined with given link structures, i.e. food-web topologies, this bioenergetic models also can be applied to food webs.

The ODE models can be either analytically solved or numerically simulated. Numerical solutions are normally obtained by simulating the models until the system reaches a steady state or an invariable pattern. The predator-prey system utilized in the second research chapter (chapter 3) is solved analytically. Due to the lack of analytical solution, the tritrophic food chain is numerically simulated. The ODE models of more complex systems with more species are normally solved by simulating as well.

\section{How global change comes into play}

\section{Physiological rates scale with species traits}

To better quantify the basic energetic principle in food webs, the physiological rates and densities mentioned above have to be given values according to the basic principles of the chemistry and physiology of organisms. The metabolic theory of ecology provides a framework combining the scalings of physiological rates and densities with two core ecological traits: body mass and temperature (Brown et al., 2004). With the aid of these scaling relationships, the metabolic theory of ecology 
allows to parameterize the ODEs of food-web models which present basic patterns of real ecosystems (e.g. Brose et al., 2006b). These scaling relationships also have rich empirical support (e.g. Brown et al., 2004; Savage et al., 2004a; Ehnes et al., 2011). For example, Meehan (2006) abstracted the scalings of natural population densities with both body mass and temperature and Ott et al. (2014) improved the scaling with body mass by using almost 5000 data points. In these „allometric equations“ which describe the scaling relationships, physiological rates scale with body-mass by a power law and with temperature by an exponential function:

$$
Y=a_{y} M^{b} e^{E \frac{T-T_{0}}{k T T_{0}}}
$$

in which $Y$ stands for a physiological rate or density (e.g. metabolic rate or a functional response parameter); $a_{y}$ is a constant; $b$ is the power law scaling with body-mass $(M)$ and $E$ stands for the exponent with temperature $(T)$. The parameters, $T_{0}$ and $k\left[\mathrm{eV} \mathrm{K}^{-1}\right.$ ], are constants converting Celsius temperature to Arrhenius temperature. The Arrhenius temperature allows to set the scaling part of temperature to unity when temperature equals to $T_{0}$ which can be set to a value based on the basic temperature of any target system. For example, $T_{0}$ was set to average German temperature in the second research chapter (chapter 3 ) to be consistent with the database where I derived the other scaling values.

\section{Parameterization}

To systematically illustrate the influence of global change on biodiversity, I tried to add another scaling relationship which depicts the scalings of functional response parameters with habitat degradation in the framework above (Eq. (1.5)). These empirically derived scalings often come from statistical analyses of certain empirical data. These meta-analyses are carried out using models of linear regression with log-transformed data. After obtaining the intercepts and estimates of regressions, they can be used in back-transformed equations (Eq. (1.5)). The intercept represents the constant $\left(a_{y}\right)$ and estimates of regressions represent the scalings ( $b$ and $E$ ). By adding another independent variable in the models of the statistical analyses which stands for habitat loss or habitat simplification, I am able to analyze the potential scaling relationship between functional response parameters and habitat degradations.

Moreover, I searched for body-mass scalings of species' behaviors (e.g. movement and predator-prey encounter) and digestive properties (e.g. gut size) and parameterized these respective traits in the individual-base model (IBM) in the second research chapter (chapter 3). These parameterizations distinguish my IBM model from others which have rather random trait values and enable the findings from my IBM model to be more convincing. 


\section{Difficulties in parameterizing the constants}

Due to lack of empirical support, earlier studies usually utilize a set of relative constants for these allometric equations. Therefore, the ODE models of a food chain or food web need to be transformed to have a set of relative constants. By transforming, the modeled system is given a relative timescale at the same time. For example, the timescale of such models is defined by setting the mass-specific growth rate of the basal species with body mass of $1 \mathrm{mg}$ at $293.15 \mathrm{~K}\left(20^{\circ} \mathrm{C}\right)$ to unity. Then, all other biological rates are normalized by the timescale according to Yodzis and Innes (1992); Otto et al. (2007):

$$
\begin{aligned}
& r=r_{0} M^{b_{r}} e^{E_{r} \frac{T-T_{0}}{k T T_{0}}}, \\
& x=x_{0} M^{b_{x}} e^{E_{x} \frac{T-T_{0}}{k T T_{0}}}, \\
& K=K_{0} M^{b_{K}} e^{E_{K} \frac{T-T_{0}}{k T T_{0}}}, \\
& B_{0}=b_{0} M_{c}^{b_{B}} A^{c_{B}} e^{E_{B} \frac{T-T_{0}}{k T T_{0}}}, \\
& f_{\max }=f_{0} M_{c}^{b_{f}} A^{c_{f}} e^{E_{f} \frac{T-T_{0}}{k T T_{0}}},
\end{aligned}
$$

with $r_{0}, x_{0}, K_{0}, b_{0}$ and $f_{0}$ as normalized constants. However, with more and more meta-analytical studies on these basic rates and densities, I was able to skip the transforming process and apply the real (absolute) timescale to my food-chain model in the last research chapter (chapter 4). Moreover, combining the meta-analytical results of a big dataset of functional responses and other meta-analytical studies, I was also able to directly apply the real spatial scale to this food-chain model by utilizing appropriate constants in these allometric equations.

\section{Outline of research chapters}

To elucidate the impact of habitat degradations on biodiversity with the association of global warming from the viewpoint of species interactions, I investigated feeding interaction strengths and its relevance to food-web motifs from both empirical and theoretical approaches in the following research chapters. I first went deeply into the feeding processes, trying to address some key points affecting the estimates of feeding interaction strength from the point of view of conducting feeding experiments (chapter 2). In the light of the gained understanding of these feeding processes, I generated an individual-based and process-based model to explore the effects of habitat loss (i.e. patch size) and habitat complexity on the estimates of feeding interaction strength (chapter 3 ). With the aid of this model, I was able to investigate very large gradients of patch sizes and habitat complexities which would be impossible for empirical studies to gain a more systematical understanding. Finally, I investigated the synergistic effects of habitat loss and warming on feeding 
interaction strength and their relevance to biodiversity by upscaling theses effects on interaction strengths to a basic food-web motif, food chain (chapter 4). 

Part II

Research chapters 



\title{
Experimental duration and predator satiation level affect functional response parameters
}

\author{
Yuanheng Li, Björn C. Rall, Gregor Kalinkat
}

\begin{abstract}
Empirical feeding studies where the feeding rates are fitted to functional response models are often used to parameterize the feeding interaction strengths in population or food web models. Whether the estimates of functional response parameters from short-term feeding studies are valid to represent the feeding interaction strengths in long-term models remains largely untested. To address this void, we utilized a meta-analytical approach to test systematic influences of experimental duration and satiation level of predator on the estimation of functional response parameters, attack rate and handling time. Meta-analyses show that attack rates decrease with increasing experimental duration and handling times for starved predators are consistently lower than handling times for satiated predators. Therefore, both the experimental duration and the predator satiation would have major influences on the predictions of the dynamics and stability of populations and food webs as the feeding interaction strengths are a key aspect in population and food web models. Our study especially highlights that theorists shall not take the functional response parameters estimated from short-term feeding experiments to parameterize population dynamics models and also take into account the influence of satiation level of predator.
\end{abstract}

Keywords | Type II functional response, Handling time, Attack rate, Experimental duration, Predator satiation level, Diurnal cycle, Digestive limit 


\section{Introduction}

Understanding species interactions and how they shape communities and ecosystems is one of the core topics in ecological research. Among different types of interactions, those that are trophic (i.e. trophic interactions) are fundamental for ecosystems, as they determine energy flow and nutrients cycling in ecological networks (Elton, 1927; Brown et al., 2004; Thompson et al., 2012). Trophic interaction strengths play a crucial role in determining population dynamics and the stability of food webs (May, 1972; Oaten and Murdoch, 1975a; Oksanen et al., 1981; Rall et al., 2008; Brose, 2010; Kalinkat et al., 2013b; Li et al., 2017a). Furthermore, the higher the stability of a food web the better it sustains biodiversity and ecological functioning (Schneider et al., 2016). Functional response models relating per capita feeding rates of consumers to resource densities (Solomon, 1949; Holling, 1959b) provide a widely applied and standardized way to quantify the interaction strengths in food webs (Berlow et al., 2004). Most empirical studies aiming to quantify interaction strengths are carried out in the laboratory. The feeding data are then fitted to a functional response model to obtain estimates of the functional response parameters. These can then be used to parameterize the interaction strengths in food web models. Hence, functional response models often serve as the connection between studies of short-term, individual-level interactions and long-term, community-level studies (e.g. Kalinkat et al., 2013b). However, the majority of such studies only investigate the feeding of an individual over a short portion of its life, from minutes (e.g. Schröder et al., 2016) to days (e.g. Buckel and Stoner, 2000). These short term estimates are then applied to studies investigating immensely longer periods that may include many generations (that may extend for hundreds of years) (Fox and Murdoch, 1978). It is largely untested, however, whether functional response parameters estimated over short periods hold for longer periods. There are some case studies on this point (e.g. Fox and Murdoch, 1978), but there do not appear to be any synthetic study. How satiated predators are before feeding studies begin also affects the estimates of functional response parameters. This is because predator satiation influences its motivation to forage (Jeschke, 2007). A satiated (i.e. well fed) predator may consume fewer prey than a starved one and this alters the functional response parameters. Therefore, we will use a meta-analytical approach to address whether and how the experimental duration and the satiation level of predator influence the estimates of functional response parameters.

The type II functional response (Holling, 1959b) is the original and most widelyapplied functional response model (Jeschke et al., 2002; Rall et al., 2012). The per capita feeding rate, $f(N)$, is formulated as a function of prey (resource) density, $N$ with two parameters, instantaneous rate of searching for prey, $a$ (often called attack rate) and handling time, $h$ : 


$$
f(N)=\frac{a N}{1+a h N}
$$

In this model, Holling (1959b) assumed that a predator spends its all time foraging, i.e. in feeding-related activities, searching for prey and processing the prey. The attack rate, $a$, describes the space (area or volume) that a predator searches per unit of time, which links to the activity of ,searching for prey'. The handling time, $h$, associating with ,processing the prey', describes the average time that a predator spends on a prey item that has been caught, i.e. subduing and ingesting. These two parameters also determine the shape of the functional response curve, where the attack rate determines the feeding rate at low prey densities and the handling time determines the limit (maximum) of feeding rate, Fig. 2.1.

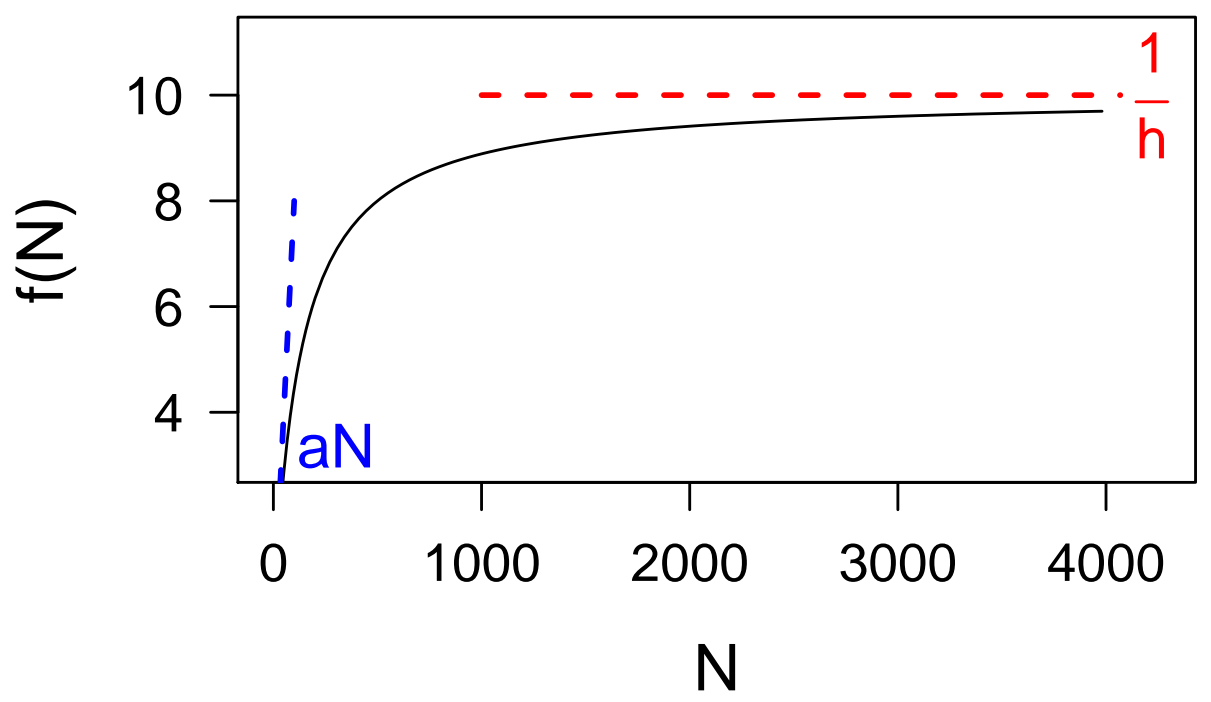

Fig. 2.1: Schematic curve of type II functional response. The red dashed line denotes the inverse of handling time, $\frac{1}{h}$ which sets the limit of maximum feeding rate. The blue dashed line denotes the tangent to the curve at minimal prey density, $N$ which describes the potential increase of feeding with prey density around low prey densities. This potential increase around low prey densities is determined by attack rate, $a$.

There is often a mismatch between the attack rates and handling times estimated by functional response models and those measured directly (e.g. by stop watch) (e.g. Mols et al., 2004; Sentis et al., 2013). This is because these parameters (attack rate and handling time), actually collapse together a plethora of biological (i.e. physiological and behavioral) processes (Jeschke et al., 2002). After all, searching for prey ${ }^{\prime}$ and ,dealing with the prey' are not the only activities in a predators life cycle, or even in its diurnal cycle (active and sleep periods). There, in fact, many more. Even when active, a predator may well not spend all its time foraging. For example, 
grazing ruminants feed intermittently rather than continuously, i.e. they switch between grazing and resting (Gregorini et al., 2006). However, the type II functional response lacks a term for such additional activities, e.g. rest or sleeping. The handling times and attack rates derived from the model thus have to incorporate the time spent on these other activities if they occur. Imagine two feeding experiments on the same predator-prey pair, one short-term (e.g. minutes) and the other long-term (e.g. days). The short-term experiment contains only foraging, but the long-term one also contains periods of predator being inactive. In consequence, the feeding rates determined from the long-term study must be lower than those from the short-term study. These reduced feeding rates in long-term experiments produce changes in the estimates of functional response parameters. They reduce estimated attack rates and increase handling times, Fig. 2.2a and 2.2b. Mathematically, the feeding rate, $f(N)$ is negatively related to the handling time, $h$ (Eq. (2.1)). Therefore, increased handling times may correspond to the reduced feeding rates estimated from long experiments (more biological reasoning below). As the attack rate derives from the average successful search rate for the entire experiment, increasing experiment duration will generally include activities than foraging which leads to reduced attack rates (Casas and McCauley, 2012).

Predator satiation influences a predator's willingness to forage (Jeschke, 2007). At the extreme, a predator with a fully-filled gut is not able to feed anymore even if its food supply is infinite. This is called ,digestive limit' (Kleiber, 1961; Herbers, 1981). It supposes that consumers are rather easily able to fill-up their guts and meet their energy needs, e.g. on a daily-base. Thereafter, the (maximum) feeding rates and therewith handling times, are also limited by gut sizes and digestion rates. Some vertebrates and invertebrates face such ,digestive limits' (Karasov and McWilliams, 2005; Jeschke and Tollrian, 2005; Jeschke, 2007). Furthermore, the satiation levels of predators are likely to strongly affect estimates of handling times (Anderson et al., 1978; Jeschke et al., 2002; Jeschke, 2007; Maselou et al., 2015). Under the assumption that ,digestive limits' apply generally to consumers, the satiation level of a predator before a feeding study will influence the estimate of handling time. Using pre-fed predators would lead to longer handling times than using starved ones. The time budget of the handling time of a satiated predator would involve not only the time for killing $\left(t_{k i l l}\right)$ and ingesting $\left(t_{i n g}\right)$, but also the time for digestion $\left(t_{d i g}\right)$, see Fig. 2.2c.

As experimental duration increases, the probability reaching satiation would increase dramatically for an efficiently foraging predator. Therefore, if the experimental duration is long enough and there is sufficient prey, the predator can reach satiation and hence face its digestive limit. In this case, the handling time could be inflated by including the time budget for digestion. As the experimental duration increases further, other activities of the predator (e.g. sleep) could be involved. In this case, increased experimental duration might lead to even larger handling times. 


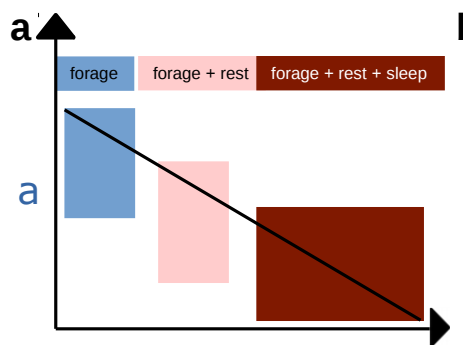

Experimental duration

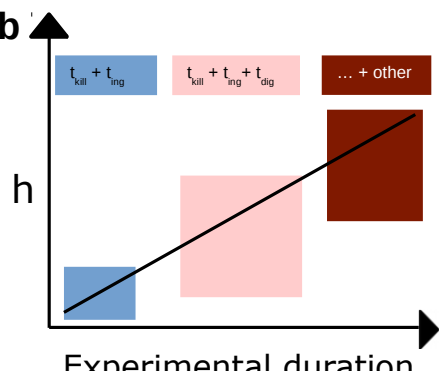

Experimental duration

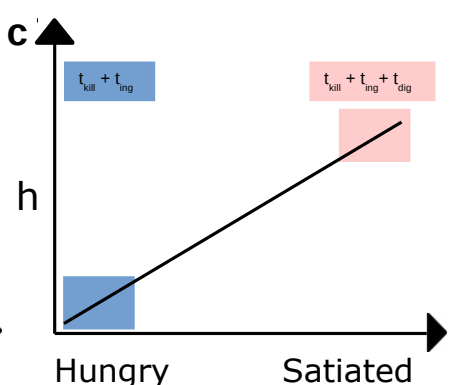

Fig. 2.2: The potential influences of experimental duration (panel $\mathbf{a}$ and $\mathbf{b}$ ) and satiation level of a predator (panel $\mathbf{c}$ ). We hypothesize that increasing experimental duration reduces attack rate, $a$ (panel a) and might also affect handling time $(h)$. Long experiments may lead to increased handling times (panel $\mathbf{b}$ ). We also hypothesize that longer handling times $(h)$ will be estimated if a satiated (pre-fed) predator is used rather than hungry (starved) predators (panel c).

In this study, we used a dataset of Type II functional responses that contained 451 data points involving 14 orders of magnitude in predator body-mass and covering predator species from 28 orders (Rall et al., 2012; Li et al., 2017b). The data derived mostly (99\%) from controlled laboratory experiments. In $78 \%$, the predators were arthropods and in $17 \%$ vertebrates. We analyzed the dataset to test (1) if the experimental duration has any effect on functional response parameters, especially on the attack rate and (2) if the influence of predator satiation on handling time holds through different taxonomies and body sizes. We address here that the functional response parameters, handling time and attack rate, are affected by both experimental duration and predator satiation. As consumers not only forage but also devote their time to other activities like rest and sleep in their diurnal cycles, (1) attack rates must decrease with increasing experimental duration and (2) handling times may also increase with increasing experimental duration. As elaborated above, we assume that, in general, satiated predators consume fewer prey than hungry ones on the premise that all other conditions are the same. The handling time evaluated for the satiated predators would incorporate not only time for killing and ingestion but also (at least) digestion. Therefore, (3) the handling time of satiated predators might be larger than that of hungry ones.

\section{Methods}

\section{Data and statistical analysis}

We used data of functional responses from empirical studies (Rall et al., 2012; Li et al., 2017b) for the meta-analyses. To this dataset, we checked and added information on the satiation levels of predators where available. Predators were considered satiated or not depending on „feeding-or-not“ before a study, i.e. ,fed“ for 
predators which were fed before the feeding trials and starved' for predators which were isolated from food source before the feeding trials. Afterwards, we excluded data points which (1) lacked information on experimental duration or predator satiation levels; (2) excluded data which are not type II functional responses and (3) excluded those for parasitoids (not suitable for testing predator satiation). The final dataset consisted of 451 data points. It included 338 data points for starved predators and 113 for fed ones. It included data on experimental duration ranging from $0.08 \mathrm{~h}$ to $240 \mathrm{~h}$ although $67.6 \%$ were for $24 \mathrm{~h}$. It also included data points for studies performed in two- and three-dimensional spaces and 243 data points were for 2D and 208 for 3D. We paid special attention to dimensionality as the units of attack rates are different in two- and three-dimensional spaces (i.e. $\left[\mathrm{m}^{2} \mathrm{~s}^{-1}\right]$ and $\left[\mathrm{m}^{3} \mathrm{~s}^{-1}\right.$ ) (Pawar et al., 2012; Li et al., 2017b).

We analyzed the functional response parameters attack rate, $a\left[\mathrm{~m}^{2} \mathrm{~s}^{-1} \mid \mathrm{m}^{3} \mathrm{~s}^{-1}\right]$, and handling time, $h[\mathrm{~s}]$ in relation to experimental duration, $t_{e}[\mathrm{~s}]$ and predator satiation, $S$ (starved, $S_{y}$ or fed, $S_{n}$ ). We additionally added predator body mass, temperature and dimensionality as explanatory variables, as they had major influences on the functional response parameters (Rall et al., 2012; Li et al., 2017b). The following equations demonstrate how we analyzed the attack rate and handling time:

$$
\begin{aligned}
& a_{2}=a_{0_{2 S}} M^{b_{2 S}} e^{E_{a_{2 S}} \frac{T-T_{0}}{k T T_{0}}} t_{e}^{i_{2 S}}, \\
& a_{3}=a_{0_{3 S}} M^{b_{3 S}} e^{E_{a_{3 S}} \frac{T-T_{0}}{k T T_{0}}} t_{e}^{i_{3 S}}, \\
& h=h_{0_{S}} M^{c_{S}} e^{E_{h_{S}} \frac{T-T_{0}}{k T T_{0}}} t_{e}^{j_{S}} .
\end{aligned}
$$

In the equations above, $a_{0}$ and $h_{0}$ are constants, $b$ and $c$ are the scaling exponents for predator body mass, $M[\mathrm{mg}], E_{a}$ and $E_{h}[\mathrm{eV}]$ are activation energies describing the exponents of temperature and $i$ and $j$ represent the scaling exponents of attack rate and handling time for experimental duration. The temperature term is transformed using Boltzmann's constant, $k$ [ $\mathrm{eV} \mathrm{K}^{-1}$ ], and the intercepts of temperature scalings are shifted to the values at $293.15 \mathrm{~K}\left(20^{\circ} \mathrm{C}\right)$ by the normalization temperature, $T_{0}$ (for more details see Gillooly et al., 2001; Rall et al., 2012). The subscript, ${ }_{S}$, represents the predator satiation which can either be starved' $\left(S_{y}\right)$ or ,fed' $\left(S_{n}\right)$. The subscripts ${ }_{2}$ and ${ }_{3}$ in the attack rate models, eq. (2.2a), (2.2b) denote the dimensionality (2D or 3D). We tested the collinearity between independent variables (Zuur et al., 2010). A variance inflation factor (VIF) test showed that there was no collinearity between any independent variables (details in the supporting information). We analyzed the data with linear mixed-effects models (,lme' function in ,nlme' package (Pinheiro et al., 2016) in R (R Core Team, 2016)) by ln-transformed data (see linear statistical models below). 


$$
\begin{aligned}
& \ln \left(a_{2}\right)=\ln \left(a_{0_{2 S}}\right)+b_{2 S} \ln (M)+E_{a_{2 S}} \frac{T-T_{0}}{k T T_{0}}+i_{2 S} \ln \left(t_{e}\right) \\
& \ln \left(a_{3}\right)=\ln \left(a_{0_{3 S}}\right)+b_{3 S} \ln (M)+E_{a_{3 S}} \frac{T-T_{0}}{k T T_{0}}+i_{3 S} \ln \left(t_{e}\right) \\
& \ln (h)=\ln \left(h_{0_{S}}\right)+c_{S} \ln (M)+E_{h_{S}} \frac{T-T_{0}}{k T T_{0}}+j_{S} \ln \left(t_{e}\right)
\end{aligned}
$$

We first selected the optimal random structures of the models based on restricted maximum likelihood (REML) and Bayesian Information Criterion (BIC) (Zuur et al., 2009, p. 121). Due to over-parameterization, the fixed structure of the attack rate model for selecting the optimal random structure, i.e. beyond optimal model (the most complex fixed structure possible, more details see Zuur et al., 2009) was selected to include all possible pairwise interactions. The beyond optimal model of the handling time model included all the possible interactions between its explanatory variables. According to the BIC, the optimal random structure of the attack rate model includes random intercept by study identity and random scaling of predator mass and the optimal random structure of the handling time model only includes random intercept which is influenced by both study identity and dimensionality. Thereafter, the best models for attack rate and handling time were selected according to BIC as well using ,dredge' function (Bartoń, 2016).

\section{Results}

Tab. 2.1: Statistical results for attack rate and handling time. All interaction terms have been excluded by model selection (see section Methods for details).

\begin{tabular}{llllll}
\hline & Variable & \multicolumn{1}{c}{$a$} & Estimate & S.E. & p-value \\
\hline \multirow{4}{*}{ attack rate } & dimension & $a_{0_{2 D}}$ & 0.78 & 1.96 & $>0.1$ \\
& predator mass & $b$ & 0.49 & 0.08 & $<0.01$ \\
& temperature & $a_{0_{3 D}}$ & -1.28 & 1.9 & $>0.1$ \\
& experimental duration & $i$ & -0.43 & 0.06 & $<0.01$ \\
& predator satiation & excluded & 0.18 & $<0.01$ \\
\cline { 2 - 6 } handling time & predator satiation & $h_{0_{S_{y}}}-0.73$ & & \\
& & $h_{0_{S_{n}}}$ & 1.64 & 0.44 & $>0.1$ \\
& predator mass & $c$ & -0.73 & 0.05 & $<0.05$ \\
& temperature & $E_{h}-0.30$ & 0.10 & $<0.01$ \\
& experimental duration & excluded & & \\
\hline
\end{tabular}

${ }^{a}$ see Eq. (2.3)

We first selected the appropriate models based on BIC for both, attack rate and handling time. The selected model for attack rate included predator body mass, temperature, experimental duration and dimensionality. The selected model for 

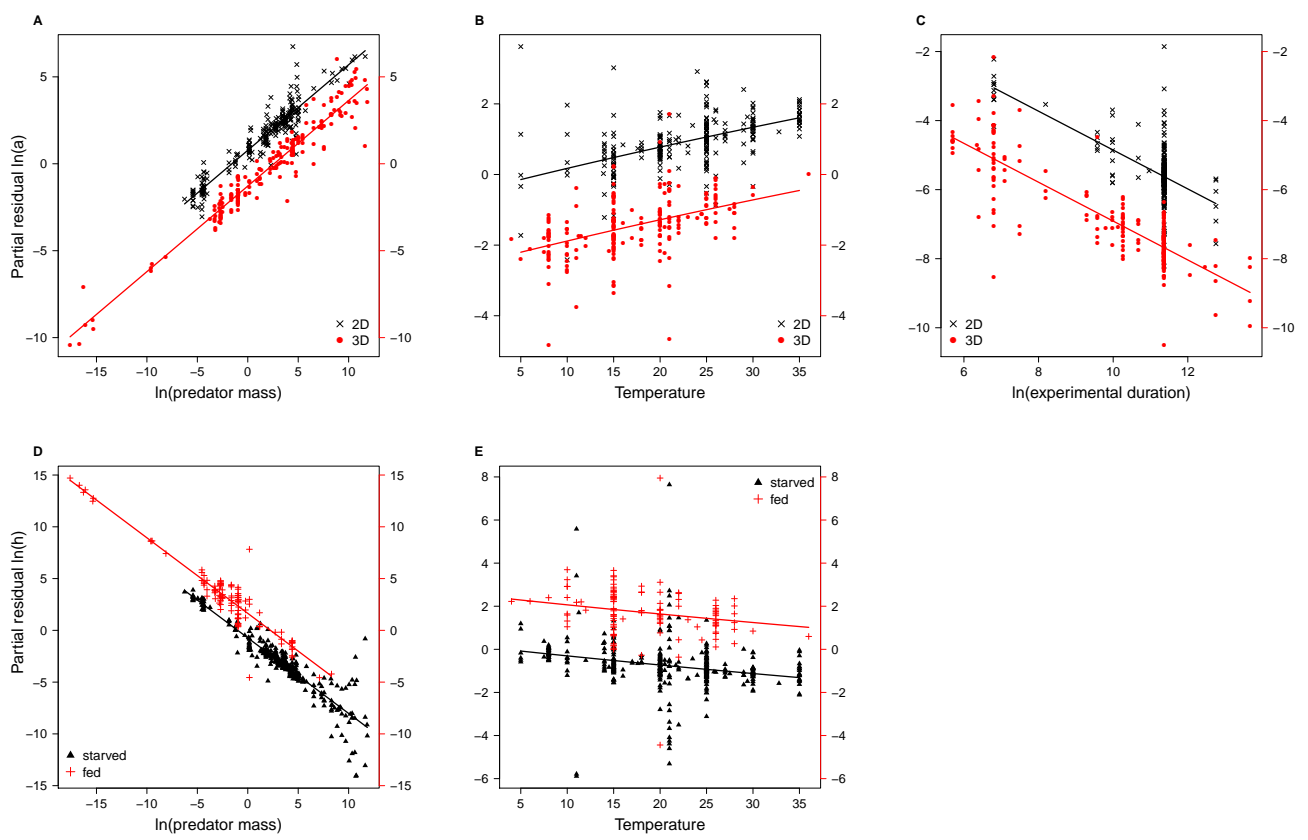

Fig. 2.3: Dependence of attack rate and handling time. Partial residuals are plotted as yaxes and all the variables other than temperature are expressed as ln-transformed. More details on the derivation of partial residuals can be found in the supporting information. The attack rates $(a)$ increase with predator body mass [ $\mathrm{mg}]$ (panel A), temperature $\left[{ }^{\circ} \mathrm{C}\right]$ (panel $\mathrm{B}$ ), and decrease with experimental duration $[\mathrm{s}]$ (panel C), while attack rates in two-dimensional settings are higher than those in three-dimensional settings (see legends and Tab. 2.1). The handling times $(h)$ decrease with predator body mass (panels D) and temperature (panels E), while handling times for fed predators are larger than those for starved predators (see legends and Tab. 2.1).

handling time included predator body mass, temperature and predator satiation (Tab. 2.1). The attack rate scaled negatively with experimental duration but not with predator satiation level. The model for attack rate included the influence of dimensionality on its intercepts even though this effect was not significant (panel A, $B$ and $C$ of Fig. 2.3). The model of handling time included the influence of predator satiation level, but experimental duration is excluded from the model. Predator satiation level did not interact with other independent variables, resulting in their only being different constants for starved and fed predators (panel D and E of Fig. 2.3).

\section{Discussion}

We used a large dataset of functional responses to investigate synthetically if the experimental duration and satiation level of predator have any effects on the estimates of functional response parameters. We chose the studies which fitted their feeding data to the most widely-spread ,type II functional response' model to gain 
more general conclusions and our integrative dataset contained data points across different ecosystem types including marine, freshwater and terrestrial and data for predators ranging from protists to vertebrates. The results showed the functional response parameter attack rate decreases with experimental duration and the handling times for satiated predators are higher than those for hungry predators. Thus two of our hypotheses were fulfilled (Fig. 2.2a, c), yet our hypothesis that experimental duration also affects handling time was rejected.

Our analyses of attack rates illustrated the influences of predator body mass, temperature and experimental duration. The results of the effects of predator body mass and temperature on attack rates are consistent with previous studies (Rall et al., 2012; Li et al., 2017b). The effect of experimental duration on the estimates of attack rates, to our knowledge, is shown for the first time from a meta-analysis. The finding that attack rate decreases with experimental duration is intuitive. Longer experimental duration will automatically mean that activities other than feeding will occur during feeding experiments. Within a diurnal cycle (24 hour period), the majority of the ,other activities' are resting and sleeping. In those cases where all other conditions are same (e.g. same predator-prey pair, same satiation level of the predator and same laboratory conditions), the estimate of attack rate of a feeding study of 24 hours will be smaller than that of an experiment of a duration only containing the active hours of the predator. This is because of the two experiments contain the same foraging but have different time lengths. Gut size can change in some predators over the long term (weekly or monthly) (Karasov and McWilliams, 2005; Van Gils et al., 2005). However, this dose not affect our results because $96 \%$ of our data are from experiments lasting $24 \mathrm{~h}$ or less. Our results thus show the effect of experimental duration alone which supported our assumption (Fig. 2.2a).

Our findings are supported by some previous studies as well. We found a study of a backswimmer (Notonecta hoffmanni) involving both short-term ( $3 \mathrm{~h}$ ) and long-term (12 h) experiments (Fox and Murdoch, 1978). Even though Fox and Murdoch did not statistically compare the estimates of functional response parameters between short- and long-term experiments, the estimated values for attack rates showed the same trend as ours. A more recent modeling study not only confirmed the influence of experimental duration on the estimates of attack rates but also explicitly highlighted the effect of involving different activities on attack rates (Casas and McCauley, 2012). For future studies, it would be important to have the opportunity to investigate whether and how even longer feeding trials, e.g. weeks and months, affect the estimates of interaction strengths (Buckel and Stoner, 2000).

Our statistical results on handling times showed the influences of predator body mass, temperature and predator satiation. With a dataset which involved both invertebrate and vertebrate predators, we showed that the estimates of handling times for starved predators were lower than those for the fed ones. Previous studies 
suggested the influence of satiation level on handling times mostly for vertebrate predators (Karasov and McWilliams, 2005; Jeschke and Tollrian, 2005; Jeschke, 2007). We generalized this finding to invertebrates, as $78 \%$ of the data are for arthropods. This indirectly supported the assumption that generally, both vertebrate and invertebrate predators face ,digestive limits'. Anderson et al. (1978), on the feeding of a fish predator (Brachydanio rerio), is the only experimental study that we are aware of to involve predator satiation. They clearly showed that starved predators had larger maximum feeding rates than satiated ones. Maselou et al. (2015) found the estimates of functional response parameters not to be affected by predator satiation. However, this study compared only between different starvation times, no satiated treatment was involved. It is the comparison between fed and starved predators, therefore, that demonstrates the effect of predator satiation level. A rather recent study investigated the influence of predator satiation with field data (Essington et al., 2000). They stated that feeding rates are reduced by predator satiation which our finding is in line with. One of their conceptual developments deserves to be more frequently considered in future studies. They separated the effect of predator satiation onto two temporal scales: (1) instantaneous satiation that occurs when feeding rate exceeds gut capacity (constraint of gut size) and (2) integrated satiation that occurs when feeding rate exceeds the time required to digest prey (constraint of digestion rate). The higher handling times associated with satiated predators may reflect mostly the constraint of digestion rate and the lower handling times of starved predators may be associated with the less strong constraint of gut size.

We switched the focus to how two common issues in feeding studies, i.e. satiation level of predators and experimental duration, affect the parameters of commonly used type II functional response model. Our study indicates that they have clear and intuitive influences on functional response parameters which are also crucial for the stability and dynamics of ecosystems and biodiversity. The increasing attack rates with decreasing experimental duration and lower handling times of starved predators will both strengthen the feeding interaction strengths in population and food web models. These changes caused by inappropriate experimental settings would strongly affect the dynamics and stability of these models. Increasing interaction strength would generally lead to stronger top-down pressure. For a predator-prey pair which has cycling dynamics, such strengthening would lead to collapse of the system and the extinction of predator species (e.g. Rip and McCann, 2011). Such strengthening may also change a stable food web to one that is dynamic and unstable (e.g. Rall et al., 2008). Therefore, to gain a relatively realistic predication of food webs, both the empiricists who conduct feeding studies on the estimates of functional response parameters and theorists who try to analyze the dynamics and stability of populations and food webs must take into account the effects of experimental duration and predator satiation. We also suggest further studies to investigate the 
relationship of species lifespan and some fundamental traits, e.g. body-mass, with the aid of which we will be able to investigate the effect of experimental duration as a portion of species life. To gain a reasonable estimate of feeding interaction strength, we suggest investigating the time period which is representable for at least the active period of the predator and report the length of its non-active period (sleeping period). For the satiation level of the predator, we suggest making trials with predators of different satiation levels. 



\section{How patch size and refuge availability change interaction}

strength and population dynamics: a combined individual- and population-based modeling experiment

Yuanheng Li, Ulrich Brose, Katrin Meyer, Björn C. Rall

\section{Abstract}

Knowledge on how functional responses (a measurement of feeding interaction strength) are affected by patch size and habitat complexity (represented by refuge availability) is crucial for understanding food-web stability and subsequently biodiversity. Due to their laborious character, it is almost impossible to carry out systematic empirical experiments on functional responses across wide gradients of patch sizes and refuge availabilities. Here we overcame this issue by using an individual-based model (IBM) to simulate feeding experiments. The model is based on empirically measured traits such as body-mass dependent speed and capture success. We simulated these experiments in patches ranging from sizes of petri dishes to natural patches in the field. Moreover, we varied the refuge availability within the patch independently of patch size, allowing for independent analyses of both variables. The maximum feeding rate (the maximum number of prey a predator can consume in a given time frame) is independent of patch size and refuge availability, as it is the physiological upper limit of feeding rates. Moreover, the results of these simulations revealed that a type III functional response, which is known to have a stabilizing effect on population dynamics, fitted the data best. The half saturation density (the prey density where a predator consumes half of its maximum feeding rate) increased with refuge availability but was only marginally influenced by patch size. Subsequently, we investigated how patch size and refuge availability influenced stability and coexistence of predator-prey systems. Following common practice, we used an allometric scaled Rosenzweig-MacArthur predator-prey model based on 
results from our in silico IBM experiments. The results suggested that densities of both populations are nearly constant across the range of patch sizes simulated, resulting from the constant interaction strength across the patch sizes. However, constant densities with decreasing patch sizes mean a decrease of absolute number of individuals, consequently leading to extinction of predators in smallest patches. Moreover, increasing refuge availabilities also allowed predator and prey to coexist by decreased interaction strengths. Our results underline the need for protecting large patches with high habitat complexity to sustain biodiversity.

Keywords | Functional response, Habitat loss, Habitat complexity, Food web, Individual-based model, Interaction strength, Population dynamics, Extinction, Patch size, Ordinary differential equation 


\section{Introduction}

The interplay between stability, complexity and biodiversity of ecological networks (especially food webs) is a subject of a long lasting and still ongoing discussion in ecology (e.g. MacArthur, 1955; May, 1972; McCann, 2000). From a mathematical point of view, an increase of species richness is only possible when the interaction strength between the species in a network (i.e. the strength of feeding interactions in a food web) decreases (May, 1972), otherwise species richness (represents biodiversity) must decline. A few possible solutions to overcome this problem have already been proposed, including a non-random organization of the feeding links in real food webs (Yodzis, 1981) and a decrease of specific interaction strength with increasing trophic levels (de Ruiter et al., 1995), both driven by allometry (Yodzis and Innes, 1992; Brose et al., 2006b; Otto et al., 2007). Classic stability analyses assumed that the strength of interactions (the functional response) increased linearly with increasing resource availability (e.g. May, 1972). In food web studies, interaction strength refers to feeding interactions, which can be studied by investigating the ,functional response': how the number of prey eaten by a predator changes with increasing prey densities. In his seminal work, Holling (1959b) described the mechanism of functional responses mathematically and showed that under the simplest assumptions it should follow a hyperbolic curve. This experiment was performed in a simple experimental trial with blindfolded students (the predator) on a plain 9 square foot table as the experimental arena and sandpaper discs as prey. Interestingly, already in the same year Holling (1959a) showed that the shape of functional response could also follow a sigmoid (i.e. s-shaped) curve when investigating small mammals on a large natural scale, including natural complexity in the habitat. The different possible shapes of functional responses are known as type I functional responses (linear with a limit), type II functional responses (hyperbolic) and type III functional responses (sigmoid), forming the core set of functional responses alongside a number of other descendant types (Jeschke et al., 2002). Notably, it has been shown that the type I functional response is an artifact (Jeschke et al., 2004; Sarnelle and Wilson, 2008), so in this study we focus only on the non-linear functional responses. One of the descendants of Holling's functional response models based on enzyme kinetics (Real, 1977) unifies the type II and the type III functional responses:

$$
f(N)=\frac{f_{\max } N^{h}}{N_{0}^{h}+N^{h}}
$$

where $f(N)$ is the per capita feeding rate, depending on the resource density, $N$. The curve is characterized by a maximum feeding rate, $f_{\max }\left[\# \mathrm{~h}^{-1}\right]$; a half saturation density, $N_{0}\left[\# \mathrm{~m}^{-2}\right]$, i.e. the prey density when the predator's feeding rate reaches half of the maximum feeding rate and a unitless Hill exponent, $h$, determining the 
curve shape. If the Hill exponent is set to unity, the resulting function is the "strict“ type II functional response. Whereas if the Hill exponent is set to two, it is the „strict“ type III functional response in a very classical sense (but see Juliano (2001) for alternative descriptions of the type III functional response). We will subsequently refer to any functional responses as type III functional responses if the Hill exponent is larger than unity.

But why is it so important to know if the functional response is a type II functional response or a type III functional response? The answer is that type III functional responses are known to stabilize population dynamics thereby allowing for persistence of large food webs (Williams and Martinez, 2004b; Brose et al., 2006b; Rall et al., 2008), by regulating prey populations to low densities (Nunney, 1980a,b). Several mechanisms have been put forward to explain why a type III functional response should appear, including the predator learning to exploit prey better (Holling, 1966) and switching between different prey types to the most abundant prey (Murdoch and Oaten, 1975; Oaten and Murdoch, 1975b). More recently, it was suggested that refuges for the prey can also lead to a type III functional response (e.g. Scheffer and De Boer, 1995; Vucic-Pestic et al., 2010a). However, there was only mixed support from studies manipulating habitat complexity in general without introducing prey refuges explicitly (Kaiser, 1983; Hoddle, 2003; Hohberg and Traunspurger, 2005; Hauzy et al., 2010; Vucic-Pestic et al., 2010a; Kalinkat et al., 2013a). Those differences may be caused by variations in how habitat complexity influences foraging and hence feeding: 1) complexity negatively affects feeding by e.g. refuges restraining predation especially at low prey densities and eventually leading to a type III functional response; 2) it affects feeding by e.g. obstacles preventing the movements of both predator and prey leading to reduced encounter rates at all prey densities but leaving the functional response type unaffected (Hauzy et al., 2010). Moreover, if the habitat complexity influences neither movement nor refuge provision, it will simply cause a dilution effect, a virtual increase of the patch size (Kalinkat et al., 2013a).

Furthermore, functional response studies are predominantly carried out under artificial laboratory conditions (Jeschke et al., 2004; Kalinkat and Rall, 2015). This means that (1) most of the studies mentioned above have used rather artificial habitat complexity and (2) due to spatial limitations of a laboratory, the size of the experimental units are relatively small (e.g. petri-dishes for estimating the functional response of ladybugs, Stethorus japonicus (Gotoh et al., 2004)). Only a few studies have attempted to investigate functional responses in natural environments, but these studies are only roughly comparable to the controlled laboratory studies as they rely on scat counting or gut content analyses combined with assessments of natural prey density (e.g. Dale et al., 1994; Smout and Lindstrøm, 2007). To our knowledge, only one study, so far, investigated and compared the simplified laboratory functional response experiments (using petri dishes) with functional responses measured in 
the greenhouse or in the field (Munyaneza and Obrycki, 1997). In this study, the attack rates in the laboratory were over 40 times higher than those in the green house but those in the field were three to nine times lower than in the petri dishes (note that all functional responses in the original publication were fitted to a type II functional response model and the attack rates were scaled to the total size of the experimental arena, we compared the attack rates scaled to square meters (Rall et al., 2012)). As the experiments carried out by Munyaneza and Obrycki (1997) altered habitat complexity and patch size at the same time, and both gradients increased simultaneously from the petri dish experiments over the greenhouse to the field experiments, it was not possible to disentangle the potential interactive effect of habitat complexity and patch size. Furthermore, Bergström and Englund (2004) reported increases in attack rates with patch size, and studies manipulating habitat complexity reported a decrease in attack rates (e.g. Vucic-Pestic et al., 2010a). This might lead to the explanation that the relatively low attack rates in the field, shown in the study of Munyaneza and Obrycki were due to the increased patch size and habitat complexity.

Beside the examples above, we are not aware of any more study addressing the effect of patch size and habitat complexity on the functional-response parameters. Moreover, most of the studies only vary habitat complexity or patch size by up to four levels (e.g. Kalinkat et al., 2013a; Bergström and Englund, 2004) and none of them systematically varied both complexity and size. This lack of studies is perhaps due to the laborious nature of functional response studies. For example, Vucic-Pestic et al. (2010b) used prey ranging from 1 up to 4000 individuals for fitting a single functional response, measuring up to 90 feeding experiments. Doubling the patch size would already lead to a maximum of 8000 individuals and a 10 times larger patch would require already a maximum of 40000 individuals.

As such extreme laboratory settings are not feasible, we developed an individualbased model (IBM) to study the effects of patch size and refuge availability on functional-response parameters. We explored full-factorial patch size and habitat complexity to disentangle effects of both variables and eventually their interactive effects on the functional-response parameters. Subsequently, we analyzed the stability of a predator-prey system depending on patch size and habitat complexity by developing a predator-prey population dynamics model which has a long standing usage and wide applicability (see section „Methods“ for details).

\section{Methods}




\section{Individual-based model of feeding interaction}

\section{Overview}

To investigate the effects of patch size and habitat complexity (represented by refuge availability) on functional-response parameters, we developed an individual-based allometric predator-prey model (for details, see „Overview, Design concepts, Detail protocol" in the supporting information, Grimm et al. $(2006,2010)$ ) to mimic the feeding experiments in the laboratory. We assumed that the maximum feeding rate was driven by mechanical and physiological processes such as chewing and digestion and would not scale with patch size or refuge availability. Therefore we first investigated the maximum feeding rate without any explicit space properties. Second, we modeled a two-dimensional square area to mimic an explicit patch in which both predator and prey can continuously move. The modeled patch consisted of cells all individuals can enter, however cells may be marked as refuges preventing predation.

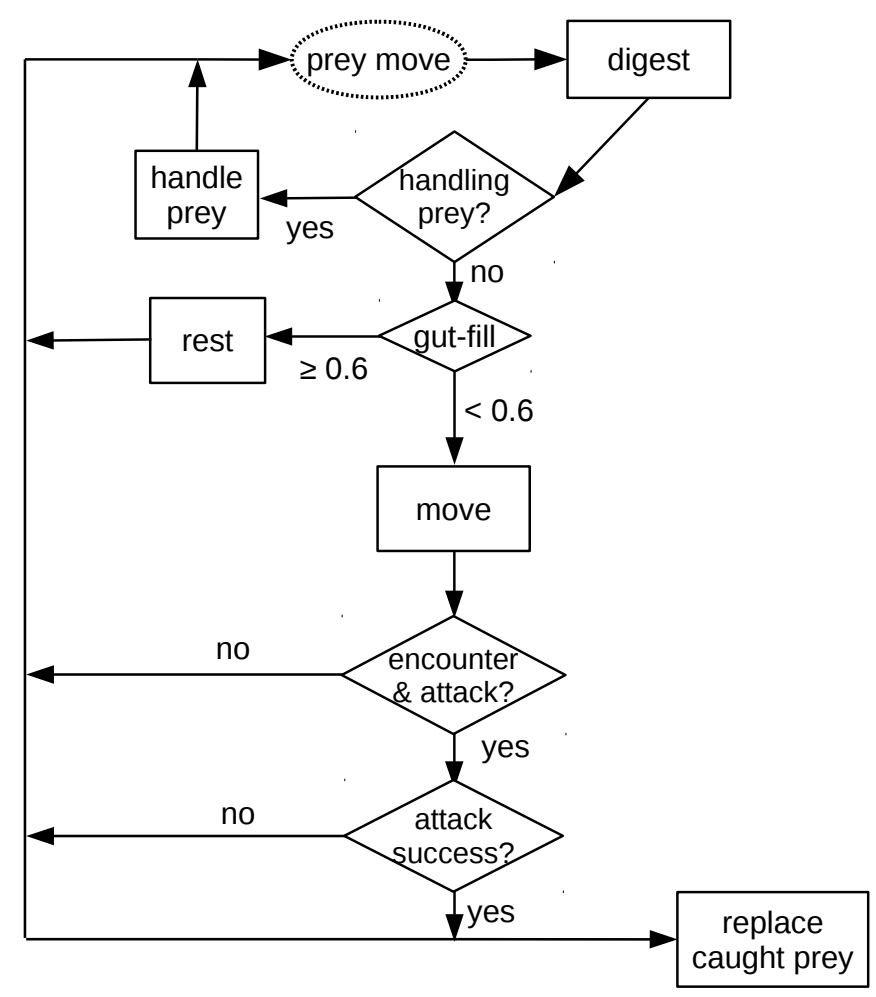

Fig. 3.1: Schematic diagram in processes of the in silico feeding experiment model. The text in the dashed oval is the only prey action which is also the start of the processes. Texts in the squares are decisions or actions of the predator. Texts in the diamonds are decisions making, e.g. ,handling prey?‘ 


\section{The Model Processes}

The first process applied in the model is prey movement (random walk with randomly chosen direction, 0 to $2 \pi$ double precision floating number and allometrically calculated distance). The following processes applied in the model are all decisions and actions of the predator (Fig. 3.1). First, the digestion of the predator is calculated. Subsequently, the algorithm checks if the predator is handling prey (caught in an earlier time step). If not and the predator's gut is full $(>=60 \%)$, it rests (not taking further actions). If the predator is not handling prey and is hungry (gut filling $<60 \%$ ), the predator moves (random walk, see above). After reaching the new position, the predator investigates if it encounters a prey in the cell. If there is a prey individual in the same cell, it will be attacked. If the attack is successful, another prey item is placed randomly into the grid to keep the prey density constant. The predator starts to handle (chew) prey in the next time step.

\section{Variables and parameters}

Most species traits regulating the processes described above follow allometric rules (Kleiber, 1961; Peters, 1983; Brown et al., 2004; Brose, 2010), including velocity, $V\left[\mathrm{~cm} \mathrm{~s}^{-1}\right]$, of both the predator and the prey (Peters, 1983); and the traits of the predator: gut size, $G$ [ $\mathrm{mg}$ ] (Ibarrola et al., 2012), digestion rate, $D$ [ $\mathrm{mg} \mathrm{s}^{-1}$ ] (Ibarrola et al., 2012), handling time, $T_{h}$ [s ] (estimated from Rall et al., 2012, see supporting information) and attack success, $S_{a}$ [unitless] (Brose et al., 2008; Gergs, 2011):

$$
\begin{aligned}
V & =v_{0} M^{a_{v}} \\
G & =g_{0} M^{a_{g}} \\
D & =d_{0} M^{a_{d}} \\
T_{h} & =h_{0} M_{p}^{a_{h, p}} M_{n}^{a_{h, n}} \\
S_{a} & =a_{0}\left(\frac{R}{R_{o p t}} e^{1-\frac{R}{R_{o p t}}}\right)^{\lambda}
\end{aligned}
$$

where $v_{0}, g_{0}, d_{0}$ and $h_{0}$ are constants, $a_{v}, a_{g}, a_{d}$ and $a_{h}$ are the allometric scalings, and $M$ is the body mass of the corresponding individual. Subscripts, ${ }_{p}$ and ${ }_{n}$ indicate predator and prey respectively. We used the widespread generalized Ricker's function (Persson et al., 1998; Persson and Brönmark, 2002b,a; Wahlström et al., 2000; Brose et al., 2008; Rall et al., 2011) to describe the scaling of attack success depending on body mass. This function consists of the maximum attack success $a_{0}$, predator-prey body-mass ratio, $R$ and its optimum $R_{o p t}$ and a shaping parameter, $\lambda$. Predator and prey also possessed some state variables to assist their decision making and 
activities, i.e. the ,position' for all individuals; the gut fullness' and if the predator is ,still handling' and an identifier, ,prey identity', to distinguish between the prey individuals.

\section{Parameters' range}

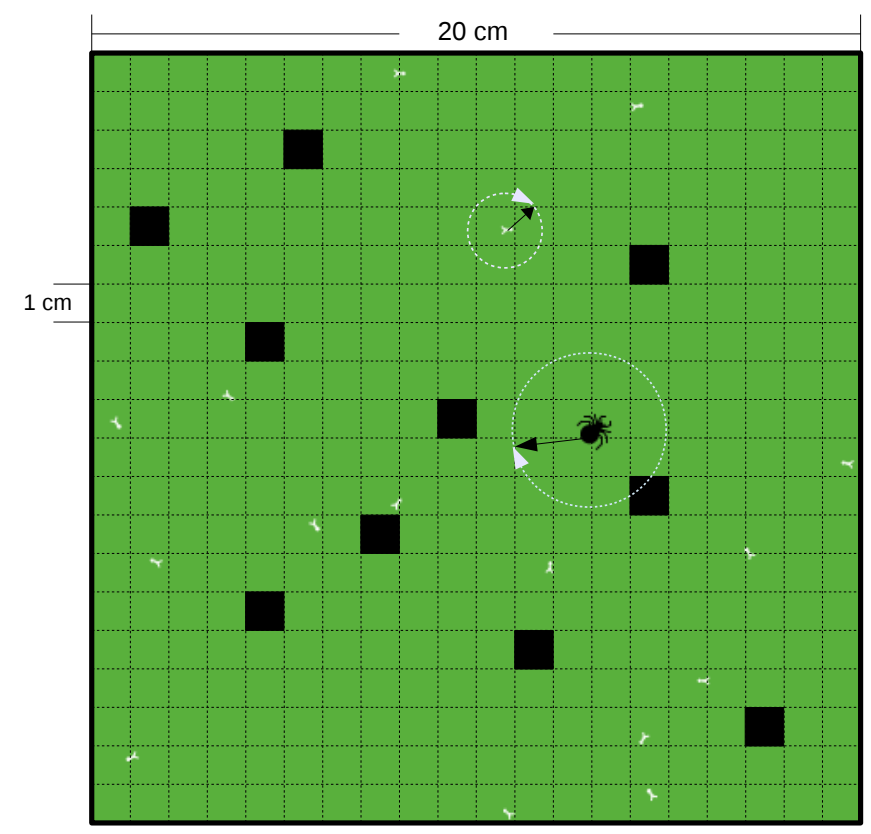

Fig. 3.2: Schematic diagram of a square grid of the IBM model. The grid in this example is $0.04 \mathrm{~m}^{2}$ with cell resolution of $1 \mathrm{~cm} \times 1 \mathrm{~cm}$ and with a,wall-boundary condition (individuals not able to penetrate the walls). The black cells are the refuge cells. The small white bugs represent prey and the big black bug represents the predator. The random walk of the individuals is decided by a randomly chosen direction, 0 to $2 \pi$ and an allometrically decided distance (as denoted by the white circles and black arrows).

The cell resolution of the square grid, in which the in silico simulations are conducted is $1 \mathrm{~cm} \times 1 \mathrm{~cm}$. As we intended to mimic laboratory experiments, the walls of the grid are set to ,wall-boundary condition' (individuals cannot penetrate the walls). We chose twelve patch sizes ranging from $0.2 \mathrm{~m} \times 0.2 \mathrm{~m}=0.04 \mathrm{~m}^{2}$ (the size of a standard patch in some terrestrial functional response experiments (Brose et al., 2008; Rall et al., 2010; Vucic-Pestic et al., 2010a; Rall et al., 2011; Vucic-Pestic et al., 2011; Kalinkat et al., 2013a) to $100 \mathrm{~m}^{2}$ (the size of a field patch (Munyaneza and Obrycki, 1997)). The sizes of each patch were: $0.04 \mathrm{~m}^{2}, 0.16 \mathrm{~m}^{2}, 0.64 \mathrm{~m}^{2}$, $1.44 \mathrm{~m}^{2}, 2.56 \mathrm{~m}^{2}, 4 \mathrm{~m}^{2}, 16 \mathrm{~m}^{2}, 36 \mathrm{~m}^{2}, 49 \mathrm{~m}^{2}, 64 \mathrm{~m}^{2}, 81 \mathrm{~m}^{2}$, and $100 \mathrm{~m}^{2}$. The second independent variable we modeled was prey refuge that served as surrogate for habitat complexity which preventing feeding. We randomly selected refuge cells on 
Tab. 3.1: Parameters values in allometric equations (Eq. 3.2)

\begin{tabular}{clcl}
\hline parameter & value & parameter & value \\
\hline$v_{0}$ & 0.546 & $a_{v}$ & 0.29 \\
$g_{0}$ & 0.50 & $a_{g}$ & 0.434 \\
$d_{0}$ & $5 \times 10^{-5}$ & $a_{d}$ & 0.75 \\
$h_{0}$ & 37.504 & $a_{h, p}$ & -0.330 \\
& & $a_{h, n}$ & 0.173 \\
$a_{0}$ & \multirow{2}{*}{0.10} & $R_{\text {opt }}$ & 100 \\
& & $\lambda$ & 1 \\
\hline
\end{tabular}

the grid for each simulation in a certain percentage of cells in steps of 5\% (5\%-75\% as the ratio of refuge cells to all cells), see Fig. 3.2 as a case example. These two independent variables are full-factorially simulated. For each simulation run, the refuge distribution is newly drawn. Those randomly chosen cells do not support any feeding by the predator and therefore act as refuges for the prey. The body masses of the predator and prey were set to $100 \mathrm{mg}$ and $1 \mathrm{mg}$, a common body-mass ratio for animal predatory interactions, close to the optimal feeding ratio of invertebrates (e.g. Vucic-Pestic et al., 2010b; Rall et al., 2011; Kalinkat et al., 2013b). We ran each of the in silico feeding trials for 3600 steps (representing $1 \mathrm{~h}$ ). The simulation for estimating the maximum feeding rate was repeated 50 times and each prey density dependent simulation was repeated five times. We simulated prey densities from $2^{0}$ to $2^{n}$ as the density when the predator (only one predator per simulation) is satiated. For example, twenty prey densities from $2^{0}$ to $2^{19}$ are selected for the patch size of $36 \mathrm{~m}^{2}$ and 35\% refuge-area ratio. Values for the parameters in allometric Eqs, Eq. (3.2), are empirically-based and given in Tab. 3.1. These values (Tab. 3.1) are derived from the same studies where we derived the formulas. Yet the maximum attack success $a_{0}$ is taken as the mean of 5 measurements from Gergs (2011). The optimum predator-prey body-mass ratio is consistent with terrestrial invertebrates from Brose et al. (2008).

\section{Statistics}

\section{Functional response fitting}

We first calculated the mean maximum feeding rate for the predator-prey pair. We used a generalized linear model (GLM) assuming that maximum feeding rates follow Poisson distribution as feeding rates were count data of non-negative integers of which the error distribution increases with increasing mean. The statistics were ran in $R$ (R Core Team, 2016), but see chapter 13 in Crawley (2007) for details. Subsequently, we used this mean maximum feeding rate as a fixed parameter in the functional response model (Eq. (3.1)) to estimate the dependencies of the remaining half saturation density and Hill exponent. 
We analyzed the feeding data from IBM models using Real's functional response, Eq. (3.1). As there is no well-established scaling relationships of functional-response parameters (half saturation density and Hill exponent) to habitat properties investigated here, i.e. patch size and refuge availability, we preliminarily tested whether the scalings of these functional-response parameters followed a power law or exponential function. To reduce the potential influences of interaction terms (between patch size and refuge availability) which may influence the dependencies of the half saturation density or Hill exponent, we included all interaction terms in the preliminary testing (Zuur et al., 2009). We analyzed in total 16 full models and compared them using the Bayesian Information Criterion (BIC), see Tab. S3.3 in the supporting information. This analysis revealed that the scalings of half saturation densities with patch size and refuge availability can be best described by a power law and an exponential function, respectively:

$$
N_{0}=C_{N_{0}} A^{a_{N_{0}}} e^{b_{N_{0}} R} e^{\gamma_{N_{0}} \ln (A) R}
$$

where $C_{N_{0}}$ is a constant, $a_{N_{0}}$ is the scaling exponent of half saturation density to patch size, $A, b_{N_{0}}$ is the scaling parameter of half saturation density to refuge availability, $R$ and $\gamma_{N_{0}}$ is the parameter giving the strength of the interaction between patch size and refuge availability. Preliminary analyses also showed that the Hill exponent depended on patch size and refuge availability both following power laws:

$$
h=C_{h} A^{a_{h}} R^{b_{h}} e^{\gamma_{h} \ln (A) \ln (R)}
$$

where $C_{h}$ is a constant, $a_{h}$ is the scaling parameter of the Hill exponent to patch size, $A, b_{h}$ is the scaling exponent of the Hill exponent to refuge availability, $R$ and $\gamma_{h}$ is the parameter giving the strength of the interaction between patch size and refuge availability.

We fitted the functional response model, Eq. (3.1) with the dependencies described above using a maximum likelihood method, ,mle2()‘ (Bolker and R Development Core Team, 2014), (see Bolker (2008) for details). As we replaced eaten prey after each feeding event (see above), we assumed that the residuals followed a negative binomial distribution. We fitted this functional response model to the data assuming a log link between data and model:

$$
\begin{aligned}
\ln \left(N_{0}\right) & =\ln \left(C_{N_{0}}\right)+a_{N_{0}} \ln (A)+b_{N_{0}} R+\gamma_{N_{0}} \ln (A) R \\
\ln (h) & =\ln \left(C_{h}\right)+a_{h} \ln (A)+b_{h} \ln (R)+\gamma_{h} \ln (A) \ln (R)
\end{aligned}
$$


i.e. we did not fit the values for the constants $C$ in equations (3.3) and (3.4), but for the intercepts in the $\ln$-transformed version $\ln \left(C_{N_{0}}\right)$ and $\ln \left(C_{h}\right)$ in Eq. (3.5). We performed a model selection using the Bayesian Information Criterion (BIC) by comparing all possible combinations of setting the parameters $a, b$ and $\gamma$ to „0“, resulting in 25 meaningful combinations (note that either $a$ or $b$ only can be excluded if the interaction term, $\gamma$ is excluded).

\section{Population dynamics model}

To investigate how patch size, $A$, and refuge availability (a measurement of habitat complexity), $R$, affect population dynamics and stability of a predator-prey system, we set up an ordinary differential equations (ODE) model. Such models were widely used to study one population (e.g. Gompertz, 1825; Verhulst, 1838) over food web motifs (e.g. Lotka, 1925; Volterra, 1926; Rosenzweig and MacArthur, 1963; Rosenzweig, 1971; Yodzis and Innes, 1992) to multi-trophic food web models (e.g. Williams and Martinez, 2004b; Binzer et al., 2016; Schneider et al., 2016) and used to predict patterns of experimental microcosms (e.g. Schneider et al., 2012; Fussmann et al., 2014) up to whole food webs (e.g. Boit et al., 2012). The ODE model describes the change in prey density [ $\left.\# \mathrm{~m}^{-2}\right], d N$, and predator density [ \# $\mathrm{m}^{-2}$ ], $d P$, over time, $d t$ (Rosenzweig and MacArthur, 1963; Yodzis and Innes, 1992; Otto et al., 2007):

$$
\begin{aligned}
\frac{d N}{d t} & =r N\left(1-\frac{N}{K}\right)-\omega \frac{f_{\max } N^{h}}{N_{0}^{h}+N^{h}} P \\
\frac{d P}{d t} & =e \omega \frac{f_{\max } N^{h}}{N_{0}^{h}+N^{h}} P-x P
\end{aligned}
$$

where the prey growth is described by logistic growth with $r\left[\mathrm{~d}^{-1}\right]$ being the intrinsic growth rate and $K\left[\# \mathrm{~m}^{-2}\right]$ being the carrying capacity. The prey are consumed by the predator following Real's functional response, Eq. (3.1), with $f_{\max }$ being the maximum feeding rate, $N_{0}$ being the half saturation density and $h$ being the Hill exponent. The predator population grows according to the functional response multiplied by the assimilation efficiency, $e$, and the effective foraging time proportion $\omega$. Moreover, it loses population density by metabolism, $x\left[\mathrm{~d}^{-1}\right]$.

We used the estimated values from the functional response fitting of our individualbased model (see above) in the ODEs. Additionally we calculated the values for carrying capacity, $K$, growth rate, $r$ and metabolism, $x$, according to empirically derived studies (Rall et al., 2010; Meehan, 2006; Savage et al., 2004a; Peters, 1983) (details described afterwards). 


\section{Equilibrium densities of the predator-prey system and extinction boundaries}

The predator-prey population model has a set of non-trivial analytical solutions, being a predator isocline

$$
N=\left(\frac{x N_{0}^{h}}{e \omega f_{\max }-x}\right)^{\frac{1}{h}}
$$

and a prey isocline

$$
P=r N^{1-h}(k-N) \frac{N_{0}^{h}+N^{h}}{k \omega f_{\max }} .
$$

After obtaining these isoclines, the equilibrium densities of predator and prey are compared with extinction boundaries $\left[\# \mathrm{~m}^{-2}\right]$. Such boundary is set to an artificial small number in the common practice of ODE models, but we explicitly set it to two individuals per patch. In cases where the predator population is not sustained, i.e. the equilibrium density is less than the extinction boundary, prey population would grow to its capacity, $N=K$ and the predator population goes extinct, $P=0$.

\section{Parameter values for the ODE}

The functional-response parameters, the maximum feeding rate, $f_{\max }$, the half saturation density, $N_{0}$ and the Hill exponent, $h$, are set according to the statistical results of the IBM simulations. We assumed that the predator foraged approximately 12 hours a day (Ebeling and Bray, 1976), therefore we added a foraging time proportion $\omega=\frac{1}{2}$. The assimilation efficiency, $e$, accounts for the proportion of food overwhelmed by the predator which can be converted to its own body mass, which is set to 0.85 , a common value for predatory consumers (Yodzis and Innes, 1992; Otto et al., 2007). The prey growth follows the logistic growth consisting of the intrinsic growth rate $r$ and the carrying capacity $K$. Together with metabolic rate of the predator, these three parameters are calculated by empirically derived equations.

$$
\begin{aligned}
K & =K_{0} M_{n}^{b_{K}} e^{\frac{E_{K}}{k T}}\left(\sigma_{0} e^{\frac{E_{\sigma}\left(T_{0}-T\right)}{k T T_{0}}}\right)^{z} e^{t l_{0}(t l-1)} \\
r & =r_{0} M_{n}^{b_{r}} e^{\frac{E_{r}}{k T}} \\
x & =\sigma c_{x} x_{0} M_{p}^{b_{x}} e^{\frac{-E_{x}}{k T}}
\end{aligned}
$$

The carrying capacity $K$ scales with body mass, $M_{n}$ (gram), environmental temperature, $T(\mathrm{~K})$, net primary production of the habitat, $\left(\sigma_{0} e^{\frac{E_{\sigma}\left(T_{0}-T\right)}{k T T_{0}}}\right)^{z}$, and the trophic level of the prey, $t l$. The values for all parameters are derived for invertebrate 


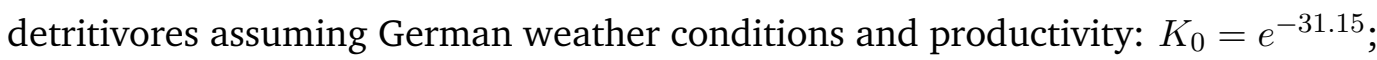
$b_{K}=-0.72 ; E_{K}=0.71 ; k=8.62 e-05 ; T=282.65 ; \sigma_{0}=600 ; E_{\sigma}=-0.35 ;$ $T_{0}=293.15 ; z=1.03 ; t l_{0}=-2.68 ; t l=1.5$ (see Meehan (2006) and Rall et al. (2010) for details). The growth rate $r$, scales with body mass (microgram) and environmental temperature, where $r_{0}=e^{32.39}, b_{r}=-0.25$ and $E_{r}=-0.84$ (details see Savage et al. (2004a) and Rall et al. (2010)). The metabolic rate $x$, also scales with body mass (gram) and environmental temperature with $x_{0}=e^{27.68}, b_{x}=0.72$ and $E_{x}=0.87$ (see Peters (1983), Savage et al. (2004b) and Rall et al. (2010) for details). Savage et al. (2004b) reported that field metabolic rate were three times larger than basal, therefore we include the coefficient $\sigma$ as 3. The normalization constant $c_{x}, 12342.86 M_{p}^{-1}$ ( $M_{p}$ in milligram), converts the metabolism from $\mathrm{J} \mathrm{s}^{-1}$ to $\mathrm{d}^{-1}$ (Peters, 1983).

We set predators to $100 \mathrm{mg}$, and prey to $1 \mathrm{mg}$, consistent with our individual-based model simulations described above. We also explored the same ranges of the patch size and habitat complexity as for the individual-based model simulations explained above. Extinction boundaries for predator and prey were set to two individuals per patch.

\section{Results}

\section{Results of IBM simulation}

Tab. 3.2: Statistical results for the in silico functional response experiments. Note that the maximum feeding rate is a priori assumed to be independent of patch size and refuge availability.

\begin{tabular}{|c|c|c|c|c|}
\hline & & Estimate & S.E. & p-value \\
\hline maximum feeding rate & $\ln \left(f_{\max }\right)$ & 1.902 & 0.05 & $<0.001$ \\
\hline \multirow{4}{*}{ half saturation density } & $\ln \left(C_{N_{0}}\right)$ & 4.577 & 0.031 & $<0.001$ \\
\hline & $a_{N_{0}}$ & -0.007 & 0.005 & 0.21 \\
\hline & $b_{N_{0}}$ & 1.777 & 0.063 & $<0.001$ \\
\hline & $\gamma_{N_{0}}$ & excluded by model selection & & \\
\hline \multirow{4}{*}{ Hill exponent } & $\ln \left(C_{h}\right)$ & 0.25 & 0.011 & $<0.001$ \\
\hline & $a_{h}$ & excluded by model selection & & \\
\hline & $b_{h}$ & excluded by model selection & & \\
\hline & $\gamma_{h}$ & excluded by model selection & & \\
\hline
\end{tabular}

The maximum feeding rate, $f_{\max }$, was estimated prior to the functional response fitting and revealed that predators of $100 \mathrm{mg}$ fed in average 6.7 prey individuals per hour (Tab. 3.2, note that the statistics were performed using a log-link function, i.e. the ln-linear feeding rate was estimated).We subsequently fitted the functional response with a fixed maximum feeding rate. Our model comparison of patch size 


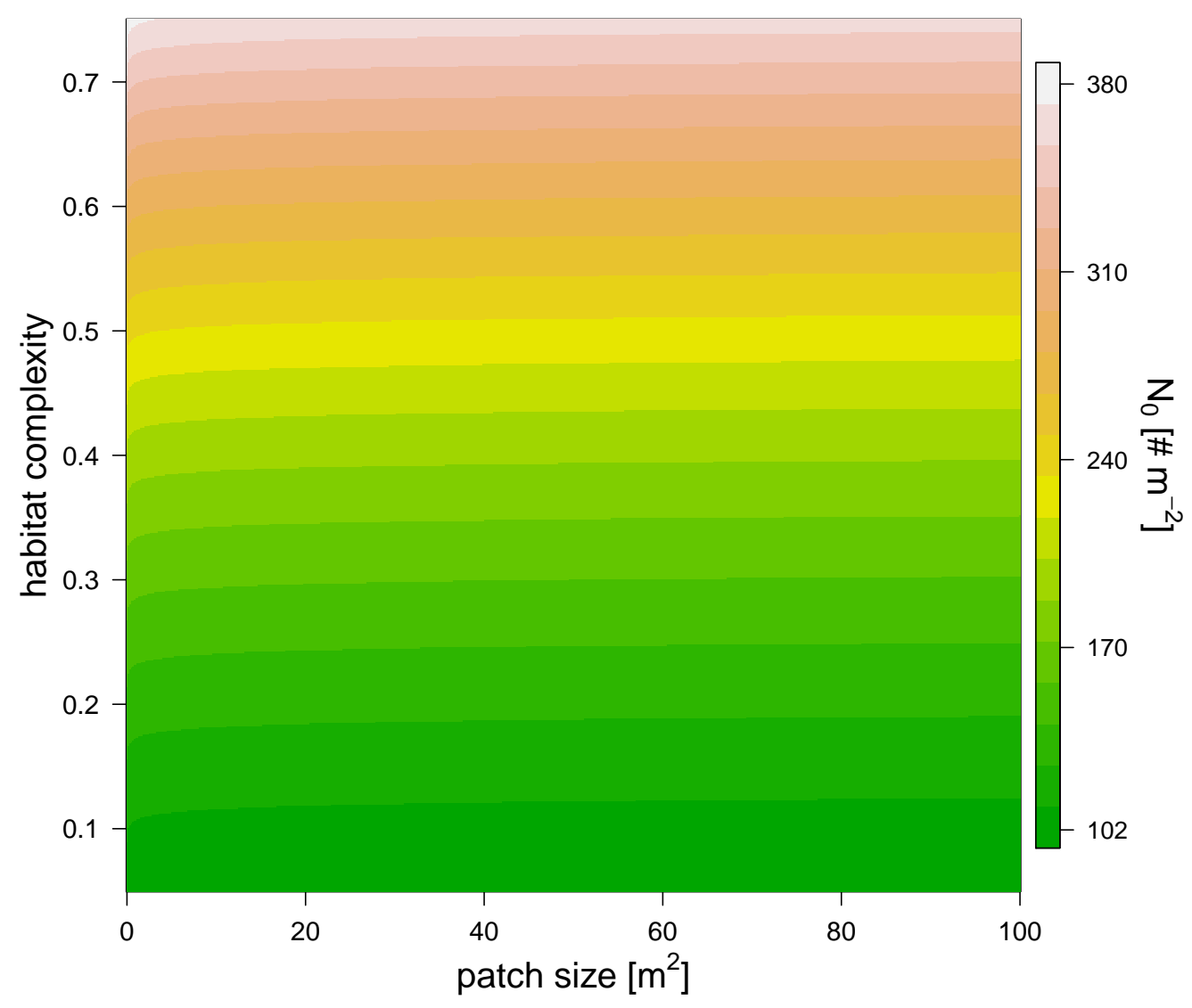

Fig. 3.3: The effect of patch size (x-axis) and refuge availability (y-axis) on half saturation density (see color scale).

and refuge availability dependent functional response models, based on BIC, included a scaling of half saturation density with patch size and refuge availability, but it did not include any scaling of the Hill exponent with either parameter (Tab. 3.2). The half saturation density increased with refuge availability, and decreased marginally with patch size, see Fig. 3.3. The estimated Hill exponent across patch sizes and refuge availabilities was 1.284 which significantly different from a Hill exponent of 1 therefore indicating a type III functional response (Tab. 3.2, note that the ln-transformed Hill exponent was tested against „0“ what is a Hill exponent of „“.). The emerging functional responses are of the same shape but feeding is realized at higher prey densities with increasing refuge availability (Fig. 3.4). Other predator-prey body-mass ratios showed similar results, see the section „In silico feeding experiments on other body-mass ratios" in the supporting information.

\section{Results of population dynamic model}

We solved the population dynamics model by a set of analytical solutions (Eq. (3.8) and (3.9)) and the extinction boundaries. In small patches only the prey species 

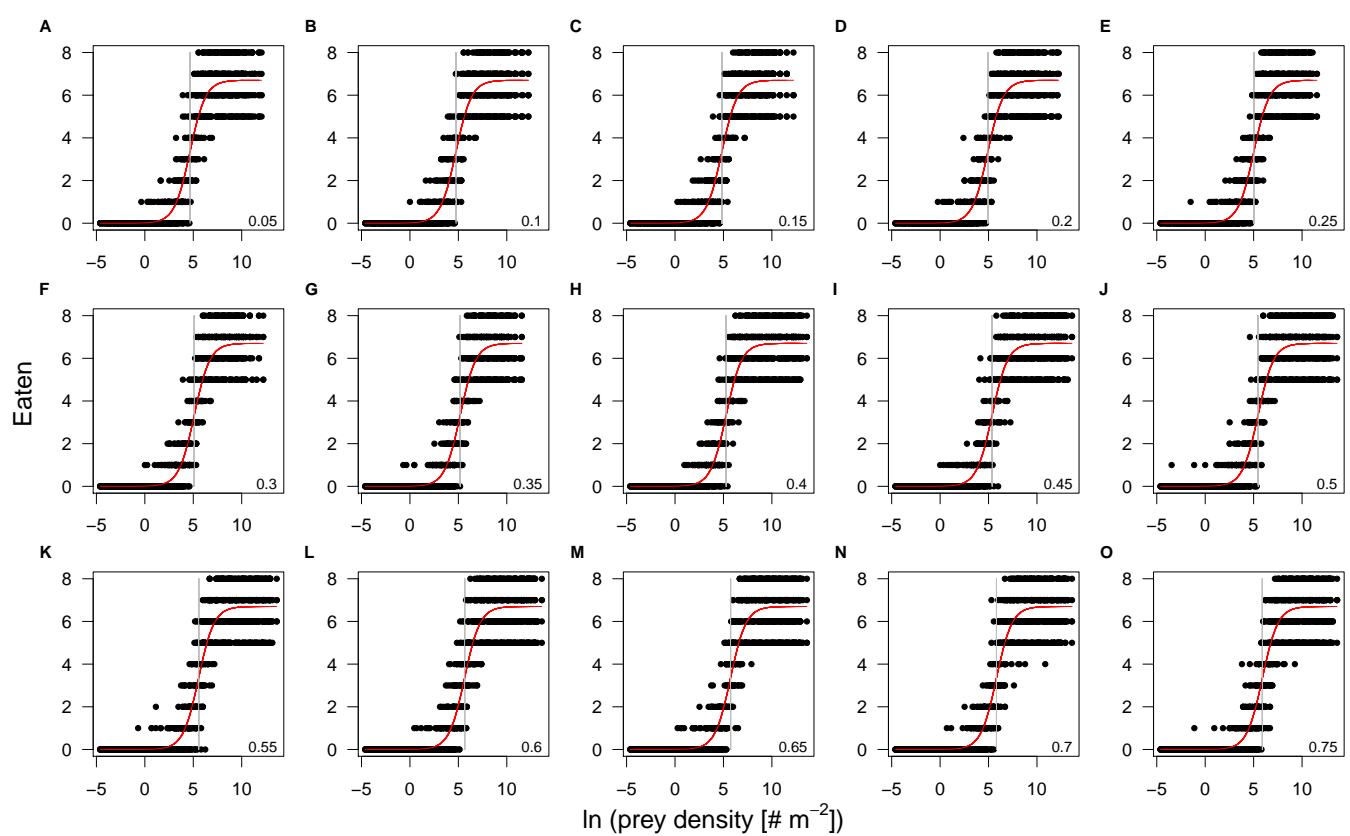

Fig. 3.4: Results of the individual-based functional response in silico experiments (black dots) and their corresponding fits (red lines). The panels are arranged by increasing refuge availability, starting at $5 \%$ (A) to $75 \%$ (O). The patch size effect on the half saturation density is too small to result in visually distinguishable regression lines. All prey densities are ln-transformed. The grey vertical lines denote the half saturation densities.

survived, but refuge availability relaxed this pattern, allowing predators to survive at smaller patches. Both predator and prey population densities increased with refuge availability, whereas in larger patches, the densities of predator and prey populations decreased slightly (Fig. 3.5).

\section{Discussion}

\section{Effects of spatial properties on interaction strength}

We developed an allometric individual-based model to investigate the effects of patch size and habitat complexity (represented by refuge availability) on feeding interactions. We found that the interaction strength decreased with refuge availability, as the half saturation density increased with it. This result is consistent with most of empirical studies aiming to account for how refuges affect predation rates (e.g. Kaiser, 1983; Folsom and Collins, 1984; Kalinkat et al., 2013a). Our results showed that patch size, however, did not significantly influence the interaction strength. Bergström and Englund (2004) reported that the attack rate (the maximum interaction strength at low prey densities) increased with patch size. This increase was explained by behavioral changes in moving activity (increasing speed of the predator) and an aggregative behavior of both their prey and predator at the walls of their experimental aquariums (animals clustered more at the aquarium walls with 

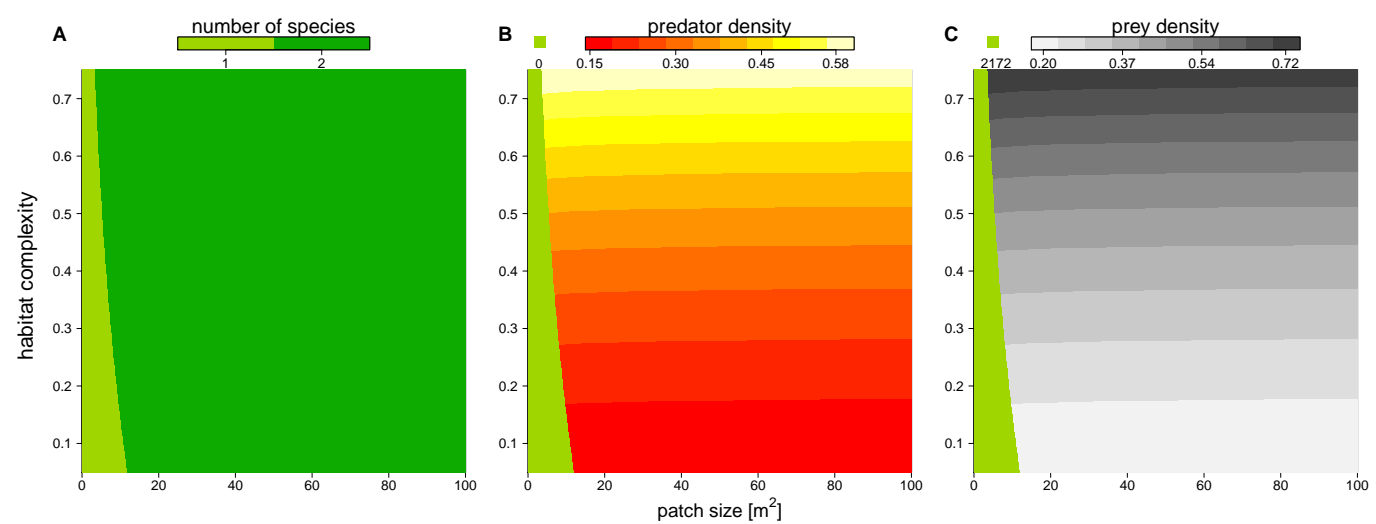

Fig. 3.5: Number of surviving species (panel A), population densities of predator (panel $\mathrm{B})$ and prey (panel C) depending on patch size ( $\mathrm{x}$-axis) and refuge availability (yaxis). When the system is embedded in very small patches, the predator becomes extinct due to energy limitation and the prey grows to its carrying capacity (green areas in $B$ and $C$ ). The non-green areas of $B$ and $C$ show the densities, $\left[\# \mathrm{~m}^{-2}\right]$, of predator and prey when the system is feasible (see color code above each plot).

increasing patch size). We did not include such behavioral changes in our model as we wanted to provide a simple basic model in this study, which may explain the differences of our results from Bergström and Englund. The Hill exponents in our in silico individual-based experiments were 1.28 across patch sizes and refuge availabilities. This is quite surprising, as a simple type II functional response was thought to be the appropriate model for feeding interaction experiments under simplified conditions in the laboratory. However, the empirical findings of Sarnelle and Wilson (2008) suggested that type III functional responses would emerge if researchers were able to include experimental trials on small prey densities, which was not feasible for experiments carried out in small patches. A few feeding interaction studies on mammals (,intelligent predators') carried out in the field also suggested type III functional responses (Holling, 1959a; Smout and Lindstrøm, 2007). More recent studies found type III functional responses for invertebrates as well (Aljetlawi et al., 2004; Vucic-Pestic et al., 2010b). Our study not only corroborates the finding of type III functional response, but also confirms that for the mechanistically simplified predators as in our individual-based model, a type III functional response is appropriate, which is not only suitable for ,intelligent predators' with the ability to learn (Holling, 1966). The statistical results for in silico experiments of other body-mass ratios showed consistency with the results discussed above.

Former laboratory experiments that compared a homogeneous habitat with a complex habitat documented a shift from a type II to a type III functional response (Vucic-Pestic et al., 2010a) and argued that this was due to a refuge effect. We did, however, not find any increase in the Hill exponent with increasing refuge availability. As we did not include explicit behaviors for hiding, we infer that this switch from a type II to a type III functional response (or an increase of the Hill exponent) not 
only needed refuges as shelter for the prey, but also active behavioral changes in sub-habitat choice (Schmitz et al., 2004; Miller et al., 2014).

Our individual-based predator-prey model framework allowed us to investigate the effects of patch size and refuge availability on functional-response parameters, which would not have been possible in laboratory or field experiments. Even without incorporating more complex movement models than random walks or behaviors like chasing or hiding, we were able to detect general patterns on the scalings of functional-response parameters with increasing patch size and refuge availability. Nevertheless, future individual based predator-prey models should incorporate more complex movement models to better understand the mechanisms of functional responses.

\section{Effects of spatial properties on population dynamics}

To investigate how changes in interaction strength scale up to population dynamics and coexistence, we analyzed a predator-prey ordinary differential equation model. We used the results from our in silico feeding experiments and combined it with empirically measured values for growth, carrying capacity and metabolism (Meehan, 2006; Savage et al., 2004a,b; Brown et al., 2004; Rall et al., 2010). Increasing patch size turned the extinction of predators to survival, meaning the smallest patches were not able to sustain the predator population. This is surprising as the feeding interaction strength does not change with patch size (i.e. a non-significant effect of patch size). As all parameters of the model are constant in respect to patch size, we expected that neither the stability (sensu population dynamics) nor persistence will be affected. This paradox behavior of the system can only be explained by the increasing extinction thresholds with decreasing patch size. We defined the extinction threshold as two individuals per patch leading to increasing densities for extinctions with decreasing patch size (Fig. 3.6 as an example). Increasing refuge availability counteracted this pattern and allowed predators to survive at even smaller patches. This is surprising as the half saturation density increased with refuge availability, suggesting less energy intake by the predator. However, increasing half saturation density also led to an increase in prey density in equilibrium that subsequently sustained a higher predator density. Both predator and prey have been feasible in larger patches and across the range of habitat complexities we explored.

Using a predator-prey population dynamics model, parameterized by the in silico functional response experiments discussed above, we were able to detect patterns of coexistence when patch size and refuge availability increased. These results are predominantly driven by the incorporation of a nearly constant feeding interactions with increasing patch size and a realistic assumption for the extinction boundaries of populations which is usually ignored in an ordinary differential equation modeling frameworks (e.g. McCann et al., 2005; Otto et al., 2007). Future studies that aim 
to investigate effects of space on persistence using ordinary differential equation models should consider to include such more realistic extinction boundaries as presented in our study.

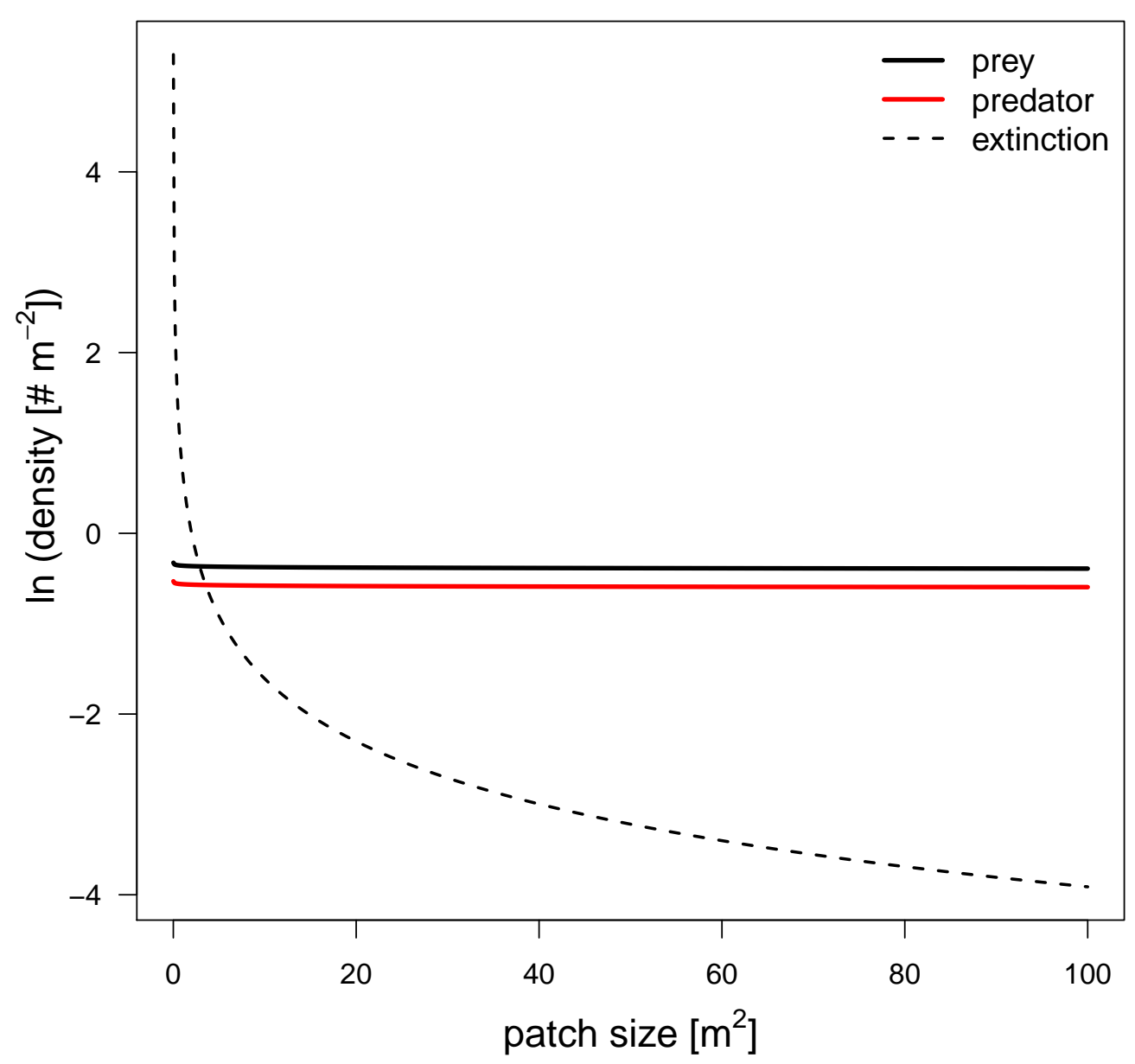

Fig. 3.6: Equilibrium densities and extinction boundaries of the predator-prey system. This is an example where $73 \%$ of the cells are prey refuges. The solid lines depict the equilibrium population densities of the predator (red) and prey. The dashed line indicates the extinction boundaries of the predator and prey, 2 individuals per patch.

\section{Conclusions}

How species interactions react to environmental changes such as habitat homogenization and habitat loss is a key point for understanding how current global changes (IPCC, 2014a) influence the stability and biodiversity of ecological networks. Increasing the stability of food webs is possible via obtaining weaker interaction strengths (May, 1972) which is important for maintaining biodiversity. We found that loss of habitat complexity would lead to increased interaction strength via 
decreasing half-saturation density. Additionally, even though the constant interaction strengths through different patch sizes lead to constant population densities, it would result in less absolute number of individuals in smaller patches. By reduced number of individuals in smaller patches, decreasing patch size would cause species extinctions, especially at higher trophic levels. Therefore, shrinking patch sizes and homogenizing habitats would both lead to destabilization of ecological networks and biodiversity loss. Altogether, our study underlines the urgent need for protecting large complex habitats to save biodiversity.

\section{Acknowledgements}

We thank Konglin Zhu, Sebastian Hanß and Daniel Ritterskamp for their help in programming, Xiaowei Wang for his technical help in computing, Gregor Kalinkat for helpful comments on the manuscript and Stephen Slocombe for his proofreading. We also thank Laith Yakob and two other anonymous reviewers for their comments that helped to improve this manuscript. 



\title{
Joint effects of habitat loss and warming alter species interactions and enhance biodiversity loss
}

\author{
Yuanheng Li, Gregor Kalinkat, Björn C. Rall
}

\begin{abstract}
Global warming and habitat loss are threatening natural ecosystems. They not only affect ecological processes and mechanisms at all hierarchical levels, but their effects are also brought into force simultaneously under current global change. While former studies predominantly focused on describing pattern changes induced by either warming or habitat loss, we shift the focus to address both mechanisms and their synergistic effects on consumer-resource interactions and food webs. We first investigated the strength of trophic interactions as it is an essential component driving the dynamics and stability of ecological networks, i.e. food webs. We studied the effects of warming and habitat loss on interaction strength by analyzing a literature based data set on consumer-resource functional responses. Thereafter, we applied the results of the meta-data analysis to a bioenergetic model of a three-species food chain to investigate their synergistic effects on population stability and species persistence. Our results indicate that habitat loss decreases the feeding rates at low resource densities by increasing half saturation densities while warming increases the overall energy intake of consumers by increasing maximum feeding rates. Even though we found an increasing energy intake of consumers with warming, energy requirements (i.e. metabolic rates) increase even more with warming leading to an reduction of energetic efficiency and consequently to an overall decreasing energy transfer to consumers with warming. Thereafter, the synergistic effects of warming and habitat loss lead to lower numbers of surviving species than either of their single effects in three-species food chains. Our study highlights that the realized threat from multiple global change drivers may be more severe than what studies on singular drivers revealed.
\end{abstract}

Keywords | Habitat loss, Global warming, Feeding, Interaction strength, Type II functional response, Food chain, Ordinary differential equation 


\section{Introduction}

Biodiversity is threatened by global environmental changes including climate change (e.g. global warming) and land-use change (e.g. habitat loss) (Sala et al., 2000; Tylianakis et al., 2008). Previous research revealed that the influence of warming permeated different levels of ecosystems, such as population dynamics (e.g. Fussmann et al., 2014), species composition and turnover (e.g. Gibson-Reinemer et al., 2015) and trophic structure (e.g. Petchey et al., 1999; Shurin et al., 2012). Especially, consumers in higher trophic levels, that are commonly larger in body mass, have a higher risk to extinct when warming (Petchey et al., 1999; Shurin et al., 2012). As warming will lead to increases of species metabolic demands (Brown et al., 2004), it, then, may lead to the increases of total energetic demands (especially) for species in higher trophic levels. Therefore, warming would lead to a higher vulnerability for higher trophic-level species than those in lower trophic levels. Besides ongoing global warming, human-induced habitat loss also affects biodiversity. Habitat loss is suggested to have large, consistently negative effects on biodiversity (Fahrig, 2003). Such negative effects are also suggested to affect species in higher trophic levels more (Holt et al., 1999; Dobson et al., 2006; Brose et al., 2016). For example, Post et al. (2000) observed shorter food-chain lengths in smaller patches. Yet we are not aware of any meta-analytical study which quantifies the effect of patch size on feeding interaction strengths or other basic biological rates.

Besides the studies on single global change drivers, there is an increasing interest among researchers to study the combined effects of multiple global change drivers on biodiversity (Tylianakis et al., 2008). IPCC (2014b) suggested that such combined effects amongst different global change drivers contributed to induce the current, rapid global change. For instance, in their current, most comprehensive metaanalytical study, Mantyka-Pringle et al. (2012) stated that the effects of habitat loss and fragmentation on general biodiversity loss (population density or species richness) were greatest in areas with high maximum temperatures yet the mechanism is lacking.

To understand the mechanisms of biodiversity loss induced by warming and habitat loss, it is of major importance to understand how species interactions are affected by these environmental changes (Schmitz et al., 2003; Tylianakis et al., 2008; Valiente-Banuet et al., 2015). However, as species and their interactions in different ecosystems respond very differently to each of the global change drivers (Tylianakis et al., 2008), exploring how warming and habitat loss affect biodiversity is not very straightforward. Therefore, investigating one species and its interactions as an integral component in the context of network structure (e.g. food web motifs) would be of crucial importance to clarify how the conjunction of these interactions affect biodiversity (Gray et al., 2014; Harvey et al., 2016). The response of one species to global change drivers is determined by the net responses of its all basic biological 
rates which quantify its energy flux and linkage to other species (Schmitz et al., 2003). These biological rates such as the feeding interaction strength (linkage to other species), growth rates (energy supply) and metabolic rates (energy demand) are cores to understand the dynamics and stability of populations, food chains up to food webs (Yodzis and Innes, 1992; Brown et al., 2004; Brose et al., 2006b).

Functional response models are a measurement of feeding interaction strengths describing the responses of feeding rates to increasing prey (resource) densities. The type II functional response model (Holling, 1959b) is one of the oldest functional response models, yet still the most utilized one. It describes the feeding rates by a hyperbolic curve (Fig. 4.1) and this curve can be mathematically captured by two functional response parameters, half saturation density $\left(B_{0}\right)$ and maximum feeding rate $\left(f_{\max }\right)$ :

$$
f(B)=\frac{f_{\max } B}{B_{0}+B}
$$

The half saturation density (biomass per area in two-dimensional patches or biomass per volume in three-dimensional patches) represents the prey density when the feeding rate of a predator reaches half of its maximum feeding rate. The maximum feeding rate depicts the maximal amount of food a predator can feed in a unit of time. In this study, we used the so-called „specific maximum feeding rate“ which quantifies the maximum feeding rate per predator mass, i.e. biomass per mass rather than biomass per individual.

The metabolic theory of ecology suggests that both feeding- and non-feeding-related rates scale with body mass and temperature (Brown et al., 2004), and can be described mathematically by:

$$
Y=a_{y} M^{b} e^{E \frac{T-T_{0}}{k T T_{0}}}
$$

in which $Y$ can stand for any of the variables mentioned above (e.g. metabolic rate, growth rate, carrying capacity or functional response parameters); $b$ is the power law scaling of body mass, $(M)$, and $E$ describes the scaling exponent with temperature, $(T)$. The parameters, $T_{0}$ and $k$ are constants converting the common-used Celsius temperature to Arrhenius temperature (see Section Methods).

Combining the metabolic theory with bioenergetic models for population dynamics (Vasseur and McCann, 2005), Binzer et al. (2012) illustrated that warming would lead to energy limitation of consumers resulting in the extinction of consumers in food chains. The energy limitation of consumers mainly comes from the inconsistent responses of energy demand and energy supply to warming (Fig. 4.2A). According to previous findings (Rall et al., 2010; Vucic-Pestic et al., 2011; Fussmann et al., 2014), for consumers, the metabolic rates (energy demand) increase with warming 


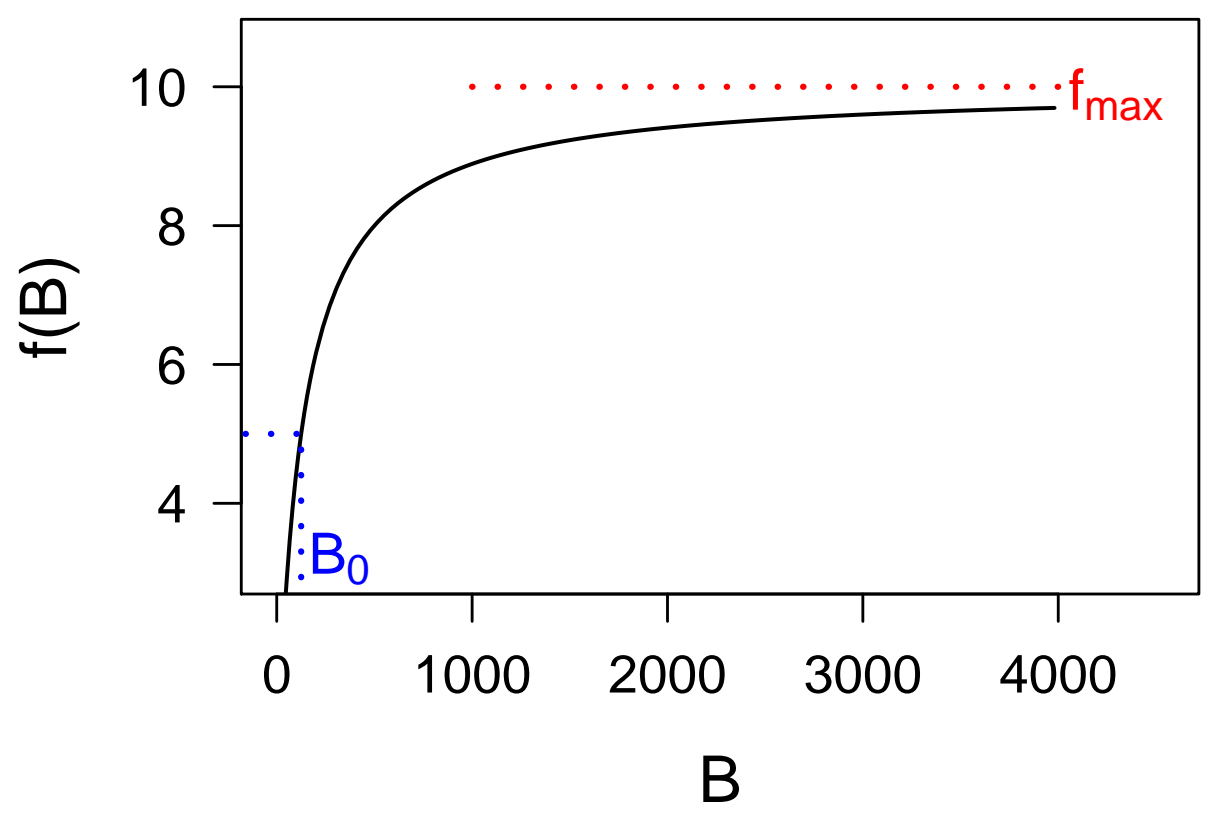

Fig. 4.1: Schematic diagram of type II functional response. The red dashed line represents maximum feeding rate $\left(f_{\max }\right)$ and the blue lines indicate half saturation density $\left(B_{0}\right)$.

more strongly than maximum feeding rates (energy gain) in general. Moreover, the carrying capacity of environment (energy supply) decreases with warming whilst the half saturation density of consumers are rather constant (Hansen et al., 1997; Vasseur and McCann, 2005; Fussmann et al., 2014). Summing up these responses subsequently results in energy limitation of the consumers and may lead to the extinction of species in higher trophic levels (Binzer et al., 2012, 2016).

Even though habitat loss is suggested to have large, negative consequence on biodiversity (see above), there does not yet exist a well-established mechanism explaining this finding. Similar to warming (above), we can build up the mechanism for habitat loss by examining the responses of those rates which determine the energy flux of species (Fig. 4.2B). Because of their physiological natures, we can assume that habitat loss does not influence the metabolic rates and maximum feeding rates of species (Brown et al., 2004; Brose, 2010). As the maximal densities of basal species (i.e. carrying capacity) are suggested to have same scalings as metabolic rates (Pawar et al., 2012), we assume that the carrying capacity does not vary with patch size either. For feeding interaction strengths, some studies suggested a hint that they shall depend on habitat loss. For example, Bergström and Englund (2004) observed that species got less active and caught less prey in smaller patches and Li et al. (2017a) also found a marginal decrease of feeding efficiency (i.e. increase of half saturation density) with increasing habitat loss. To our knowledge, comprehensive studies, generalizing such findings are yet lacking. Therefore, we speculate a case 


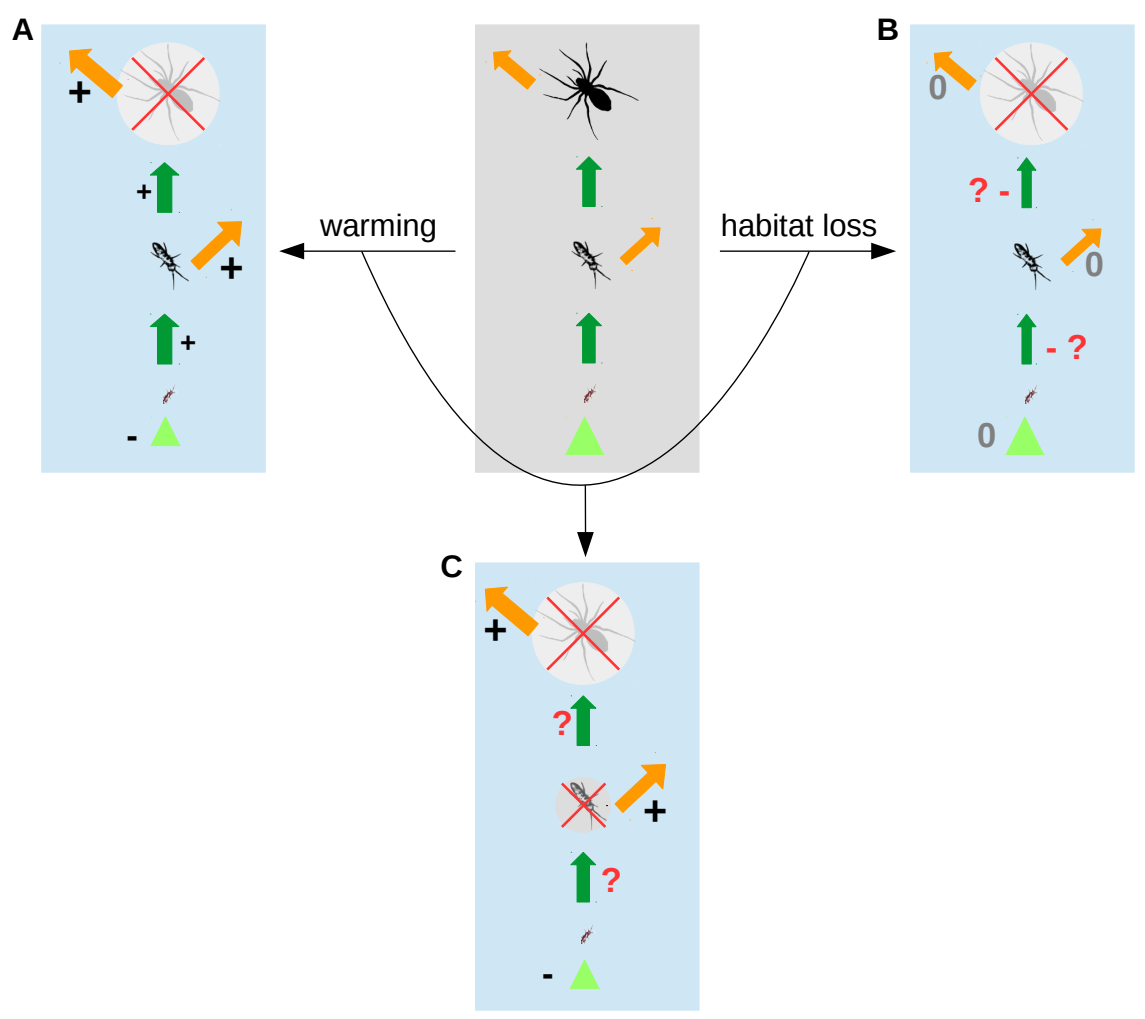

Fig. 4.2: Schematic diagram illustrating the hypotheses in this study. The gray panel in the middle shows an example of a body-mass structured food chain (e.g. predatorprey body-mass ratio as $100: 1)$. The light green triangle indicates the energy supplied to basal species (carrying capacity of the environment), the dark green arrows interaction strengths (feeding rates), the orange arrows metabolic rates. Warming would decrease the energy supply, increase both interaction strengths and metabolic rates, but increase metabolic rates more than interaction strengths (panel A on the left). As a consequence, warming causes the loss of top predator. As former studies found that habitat loss would especially harm higher level species and it would not influence energy supply or metabolic rates, the reason why habitat loss harms top predator may be that it decreases interaction strengths (panel B on the right). Therefore, the combined effects of warming and habitat loss probably would also influence the intermediate consumer.

to explain how habitat loss may induce biodiversity loss. In cases where habitat loss increases the half saturation density (decrease of feeding efficiency), habitat loss would decrease the energy gains of the consumers, leading to reduced sizes of consumer populations and eventually species loss. Based on the statements above, we could infer how warming and habitat loss synthetically affect biodiversity. As Mantyka-Pringle et al. (2012) stated a more sever negative effect of habitat loss on biodiversity in cases of higher temperature, we speculate that the energy limitation caused by warming would be enhanced by habitat loss due to its effect on restricting feeding efficiency (Fig. 4.2C). 
We attempt to answer the question how the synergy of habitat loss and warming affects biodiversity by investigating the responses of feeding interaction strengths and food chains. Previous studies showed that warming would in general restrict the biomasses of basal species as it decreases the carrying capacity and it increases metabolic rates more than increasing feeding rates (Brown et al., 2004; Rall et al., 2012). Yet, the mechanism of how habitat loss induces biodiversity loss is yet not clear. Based on the statements above, we assumed that habitat loss does not influence metabolic rates or carrying capacities (Brown et al., 2004; Pawar et al., 2012) but it would decrease the feeding efficiencies of consumers. Synthetically, we hypothesize that warming would increase feeding interaction strengths and increase the vulnerabilities of consumers in food chains. In this study, we investigated how warming and habitat loss affect functional response parameters by a meta-analysis and developed a simulation model in the light of empirically-supported parameter values to explore the synergistic effect of global warming and habitat loss on food chains.

\section{Methods}

\section{Meta-data and statistical analysis}

We used data on functional responses originally compiled by Rall et al. (2012). Before the statistical analyses, we did a quality check and added patch sizes information for all the data points according to the original papers. We only chose predation or filtration data with explicit patch sizes fitted to type II functional responses. As there are some data points which have extremely large or small patch sizes, we excluded all studies with a patch size smaller or larger than twice the standard deviations of mean patch size, to avoid the scaling of patch size to be driven by these extremes (17 data points out of 589, see Fig. S4.1). We analyzed the dependencies of half-saturation densities, $B_{0}\left[\mathrm{mg} \mathrm{m}^{-2} \mid \mathrm{mg} \mathrm{m}^{-3}\right]$, and specific maximum feeding rates, $f_{\max }\left[\mathrm{mg} \mathrm{d}^{-1} \mathrm{mg}^{-1}\right]$ on the predator body mass, $M_{c}$ [mg], environmental temperature, $T[\mathrm{~K}]$, patch size, $A\left[\mathrm{~m}^{2} \mid \mathrm{m}^{3}\right]$, the interaction between temperature and patch size and the dimensionality of the feeding interaction, $D$ (two- or three-dimensional spaces, $2 D / 3 D$ ). Prey body mass was not used as it was highly correlated with predator body mass, Pearson's $r=0.74$, p-value $<0.01$ (Zuur et al., 2007) and predator body mass was commonly used in such analysis (e.g. Brose et al., 2006b). To prevent further collinearity, we analyzed the variance inflation factors (VIF) for all the independent variables and the results showed no evidence for collinearity in these variables (for details see supporting information). The dependencies of half saturation density and specific maximum feeding rate described above can be described by (Brown et al., 2004; Rall et al., 2012): 


$$
\begin{gathered}
B_{0}=a_{B_{D}} M_{c}^{b_{B_{D}}} e^{E_{B_{D}} \frac{T-T_{0}}{k T T_{0}}} A^{c_{B_{D}}} e^{\gamma_{B_{D}} \ln (A) \frac{T-T_{0}}{k T T_{0}}} \\
f_{\text {max }}=a_{f_{D}} M_{c}^{b_{f_{D}}} e^{E_{f_{D}} \frac{T-T_{0}}{k T T_{0}}} A^{c_{f_{D}}} e^{\gamma_{f_{D}} \ln (A) \frac{T-T_{0}}{k T T_{0}}}
\end{gathered}
$$

in which $a_{B}$ and $a_{f}$ are constants, $b_{B}$ and $b_{f}$ are scaling exponents of predator body mass, $E_{B}$ and $E_{f}[\mathrm{eV}]$ are activation energies describing the scaling exponents with temperature, $c_{B}$ and $c_{f}$ represent the scaling exponents with patch size and $e^{\gamma_{B}}$ and $e^{\gamma_{f}}$ quantify the interacting effect of temperature and patch size of half saturation density and specific maximum feeding rate, respectively (i.e. the interaction terms). The subscript ${ }_{D}$ represents fits using either two- or three-dimensional spaces. $T_{0}$ $[\mathrm{K}]$ is the normalization temperature, $293.15 \mathrm{~K}$ and $k\left[\mathrm{eV} \mathrm{K}^{-1}\right]$ is the Boltzmann constant. Before the analyses, we ln-transformed the Eqs. (4.3):

$$
\begin{aligned}
& \ln \left(B_{0}\right)=\ln \left(a_{B_{D}}\right)+b_{B_{D}} \ln \left(M_{c}\right)+E_{B_{D}} \frac{T-T_{0}}{k T T_{0}}+c_{B_{D}} \ln (A)+\gamma_{B_{D}} \ln (A) \frac{T-T_{0}}{k T T_{0}}, \\
& \ln \left(f_{\text {max }}\right)=\ln \left(a_{f_{D}}\right)+b_{f_{D}} \ln \left(M_{c}\right)+E_{f_{D}} \frac{T-T_{0}}{k T T_{0}}+c_{f_{D}} \ln (A)+\gamma_{f_{D}} \ln (A) \frac{T-T_{0}}{k T T_{0}}
\end{aligned}
$$

Thereafter, we used linear mixed-effect models to analyze the data with Eqs. (4.4) by ,lme' function in the package of ,nlme' (version 3.1-128, Pinheiro et al., 2016) in $\mathrm{R}$ (version 3.3.1, R Core Team, 2016). We chose the optimal random structure using restricted maximum likelihood (,REML') and Bayesian Information Criterion (BIC) following Zuur et al. (2009, p. 121), more details of which found in the supporting information. Based on BIC, we allowed a random intercept $(a)$ and a random slope of predator body mass (b) by study identity as the random factors for the model of half-saturation density, Eq. (4.4a) and only a random intercept with the factor study identity in the model of specific maximum feeding rate, Eq. (4.4b). After choosing the random structures, maximum likelihood (,ML') estimation method (Zuur et al., 2009) were applied for obtaining the optimal fixed-structure. We chose the models with the lowest BIC values by applying an automated model selection tool, 'dredge' function in ,MuMIn' package (Bartoń, 2016). As the dimensionality defines the units of the patch sizes, e.g. $\mathrm{m}^{2}$ for $2 \mathrm{D}$, we fixed the variable dimensionality to be part of the optimal model for half-saturation densities. To get reliable p-values, we applied ,REML' to the final models (Zuur et al., 2009).

\section{Food-chain model}

We constructed a three-species food-chain model to investigate the effects of patch size and environmental temperature on population dynamics and stability (Vasseur and McCann, 2005; Binzer et al., 2012). The biomass changes of basal species $\left[\mathrm{mg} \mathrm{m}^{-2} \mid \mathrm{mg} \mathrm{m}^{-3}\right], \dot{B}_{i}$, intermediate consumer $\left[\mathrm{mg} \mathrm{m}^{-2} \mid \mathrm{mg} \mathrm{m}^{-3}\right], \dot{B}_{j}$ and top 
predator $\left[\mathrm{mg} \mathrm{m}^{-2} \mid \mathrm{mg} \mathrm{m}^{-3}\right], \dot{B}_{k}$, are described by ordinary differential equations (ODE):

$$
\begin{aligned}
& \dot{B}_{i}=r B_{i}\left(1-\frac{B_{i}}{K}\right)-\frac{f_{\max _{j}} B_{i}}{B_{0_{i}}+B_{i}} B_{j}, \\
& \dot{B}_{j}=e_{j} \frac{f_{\text {max }_{j}} B_{i}}{B_{0_{i}}+B_{i}} B_{j}-x_{j} B_{j}-\frac{f_{\text {max }_{k}} B_{j}}{B_{0_{j}}+B_{j}} B_{k}, \\
& \dot{B_{k}}=e_{k} \frac{f_{\max _{k}} B_{j}}{B_{0_{j}}+B_{j}} B_{k}-x_{k} B_{k} .
\end{aligned}
$$

The growth of basal species, $i$, is described by the logistic growth with an intrinsic growth rate, $r$ [mg d ${ }^{-1} \mathrm{mg}^{-1}$ ], and a carrying capacity, $K\left[\mathrm{mg} \mathrm{m}^{-2} \mid \mathrm{mg} \mathrm{m}^{-3}\right]$. The basal species is consumed by the intermediate consumer, $j$, which gains biomasses by this feeding interaction with an assimilation coefficient, $e_{j}$. The intermediate consumer loses biomass via feeding by the top predator, $k$ and the metabolic demand, $x\left[\mathrm{mg} \mathrm{d}^{-1} \mathrm{mg}^{-1}\right]$. The top predator gains biomass by feeding the intermediate consumer (with an assimilation coefficient, $e_{k}$ ) and loses biomass via metabolism.

The non-feeding biological rates, intrinsic growth rate $(r)$, environmental carrying capacity $(K)$ and metabolic rate $(x)$ all scale with body mass and temperature (Savage et al., 2004a; Meehan, 2006; Brown et al., 2004). The feeding-related rates, half-saturation densities of consumers, $B_{0}$, and specific maximum feeding rates, $f_{\text {max }}$, scale with body mass, temperature and/or patch size according to our statistical results:

$$
\begin{aligned}
& r=a_{r} M_{i}^{b_{r}} e^{\frac{E_{r}}{8.62 \times 10^{-5} T},} \\
& x=a_{x} M_{j / k} b_{x} e^{\frac{E_{x}}{8.62 \times 10^{-5} T}}, \\
& K=a_{K} M_{i}^{b_{K}} e^{E_{K} \frac{T-T_{0}}{k T T_{0}}}, \\
& B_{0}=a_{B} M_{j / k}^{b_{B}} A^{c_{B}} e^{E_{B} \frac{T-T_{0}}{k T T_{0}},} \\
& f_{\text {max }}=a_{f} M_{j / k}^{b_{f}} A^{c_{f}} e^{E_{f} \frac{T-T_{0}}{k T T_{0}}},
\end{aligned}
$$

where $a_{r}, a_{x}$ and $a_{K}$ are allometric constants, $b_{r}, b_{x}$ and $b_{K}$ are the scaling exponents of body masses, $E_{r}, E_{x}$ and $E_{K}$ are activation energies describing the scalings with temperature and parameters in equations of half saturation density $\left(B_{0}\right)$ and specific maximum feeding rate $\left(f_{\max }\right)$ are according to Eq. (4.3). Inserting these scaling equations (Eq. (4.6)) into the ordinary differential equations (Eq. (4.5)) yields a population-dynamic model for three-species food-chain.

Some parameter values of intrinsic growth rate and metabolic rate, $a_{x}=1.06 \times 10^{11}$, $b_{r}, b_{x}=-0.30$ and $E_{r}, E_{x}=-0.69$ are from Ehnes et al. (2011) and some values of carrying capacity, $a_{K_{2 D}}=30.20, b_{K}=0.32$ and $E_{K}=-0.71$ are from Ott et al. (2014) and Meehan (2006). To be noticed, the scalings of carrying capacity shall 
be invariant between different dimensions as it reflects the maximal abundance of primary producers (Pawar et al., 2012). For the simulations in two-dimensional spaces, the constant of intrinsic growth rate, $a_{r_{2 D}}$ is set to be proportional to the constant of metabolic rate $4.76 \times 10^{11}\left(a_{r_{2 D}}=a_{x} / 0.2227\right)$ according to Otto et al. (2007). The body masses of basal species, $M_{i_{2 D}}$, intermediate consumers, $M_{j_{2 D}}$ and top predators, $M_{k_{2 D}}$ are set to $1 \mathrm{mg}, 10 \mathrm{mg}$ and $100 \mathrm{mg}$ according to the mean predator-prey body mass ratio for invertebrates (Brose et al., 2006a) and the assimilation efficiencies are 0.85 (Yodzis and Innes, 1992) (as the basal species here is assumed to be an animal in two-dimensional cases). For the simulations in threedimensional spaces, the constant of intrinsic growth rate, $a_{r_{3 D}}$ is set to $3.37 \times 10^{11}$ $\left(a_{r_{3 D}}=a_{x} / 0.314\right)$ according to the proportionality in Rall et al. (2008); the constant of carrying capacity, $a_{K_{3 D}}$ is set to 5370.32 according to Boit et al. (2012) and Bundy (2004) and the body masses are set to $M_{i_{3 D}}=1 \times 10^{-6} \mathrm{mg}, M_{j_{3 D}}=1 \times 10^{-3} \mathrm{mg}$ and $M_{k_{3 D}}=1 \mathrm{mg}$ according to the body masses in lakes (Boit et al., 2012). The assimilation efficiency for intermediate consumer, $e_{j}$ is 0.45 (Rall et al., 2008) (as the basal species here is assumed to be phytoplankton) and that for top predator $\left(e_{k}\right) 0.85$.

We varied the patch size $\left(0.01 \mathrm{~m}^{2} \mid \mathrm{m}^{3}\right.$ to $\left.10 \mathrm{~m}^{2} \mid \mathrm{m}^{3}\right)$ and environmental temperature $\left(0{ }^{\circ} \mathrm{C}\right.$ to $\left.40^{\circ} \mathrm{C}\right)$ of the system to investigate the effect of different combinations of these two variables. To be noticed, the range of patch sizes is set according to the range in the dataset of functional response to ensure the validation of the scalings with patch size. The initial biomass densities of species were all set to half of the carrying capacity of the particular combination of patch sizes and temperatures ( $K$ in Eq. (4.6c)) and each simulation was ran for 274 years (100000 steps) to make sure the system running to an equilibrium stage. During the simulations, as long as the population biomass of a species was dropped below $1 \times 10^{-30} \mathrm{mg} \mathrm{m}^{-2} \mid \mathrm{mg} \mathrm{m}^{-3}$, it counted as death. We recorded species biomasses of last $10 \%$ of each simulation to test the stabilities of the surviving species with coefficient of variation (CV).

\section{Results}

\section{Statistical analyses of functional response parameters}

The dataset used for the statistical analyses consists of 572 observed values for half saturation densities and specific maximum feeding rates $\left(N_{2 D}=297 ; N_{3 D}=275\right)$. The best model for half saturation density included the scalings with body mass and patch size and the model for specific maximum feeding rate included the scalings with body mass and temperature (Tab. 4.1). The analyses showed that the halfsaturation densities in both two- and three-dimensional cases increased with predator mass and decreased with patch size (Fig. 4.3A and 4.3B). The specific maximum feeding rates decreased with predator mass (Fig. 4.3C), whilst they increased with 
Tab. 4.1: Statistical results for the meta-analysis. The table shows the statistical results fitting Eq. (4.4) to the dataset of functional response. The parameters that have been excluded by the model selections are marked. The constants are represented by $a$, the scalings with body mass and temperature are represented by $b$ and $E$ and the scalings with patch size are represented by $c$.

\begin{tabular}{lllll}
\hline & & Estimate & S.E. & p-value \\
\hline & $\ln \left(a_{B_{2 D}}\right)$ & 3.446 & 0.939 & $<0.001$ \\
& $\ln \left(a_{B_{3 D}}\right)$ & 4.355 & 1.068 & $<0.001$ \\
& $b_{B_{2 D}}$ & 0.500 & 0.086 & $<0.001$ \\
half saturation density & $b_{B_{3 D}}$ & 0.217 & 0.084 & $<0.01$ \\
& $c_{B}$ & -0.369 & 0.130 & $<0.01$ \\
& $E_{B}$ & excluded by model selection \\
& $\gamma_{B}$ & excluded by model selection \\
\cline { 2 - 5 } maximum feeding rate $\left(a_{f_{2 D}}\right)$ & 1.804 & 0.437 & $<0.001$ \\
& $\ln \left(a_{f_{3 D}}\right)$ & -1.027 & 0.425 & $<0.05$ \\
& $b_{f}$ & -0.248 & 0.038 & $<0.001$ \\
& $E_{f}$ & 0.274 & 0.084 & $<0.01$ \\
& $c_{f}$ & excluded by model selection \\
& $\gamma_{f}$ & excluded by model selection \\
\hline
\end{tabular}

temperature (Fig. 4.3D). The specific maximum feeding rates did not scale with patch size and the intercepts were different for two- and three-dimensional cases (Tab. 4.1). The interacting effects between temperature and patch size for both functional response parameters were excluded by model selections.

\section{Food-chain model}

When patches were small, the number of surviving species decreased with increasing temperature, but increasing patch sizes could compensate the decease of survival number of species (Fig. 4.4). However, in three-dimensional cases, such compensation effect of increasing patch sizes was minor when temperature was low (upper-left corner in Fig. 4.4B). In two-dimensional cases, all three species survived for large portions of the combination of patch sizes and environmental temperatures we explored. Yet in three-dimensional cases, the decrease of surviving number of species with increasing temperature was so quickly that only a some portion of the investigated combinations ended up to have three species survived. The results of coefficient of variation (CV) suggested that all populations for surviving species in two-dimensional cases had small CVs (upper row in Fig. 4.5). Yet for three-dimensional cases, when all three species survived, their populations had rather large CVs compared to the cases in two-dimensional spaces (lower row in Fig. 4.5). 

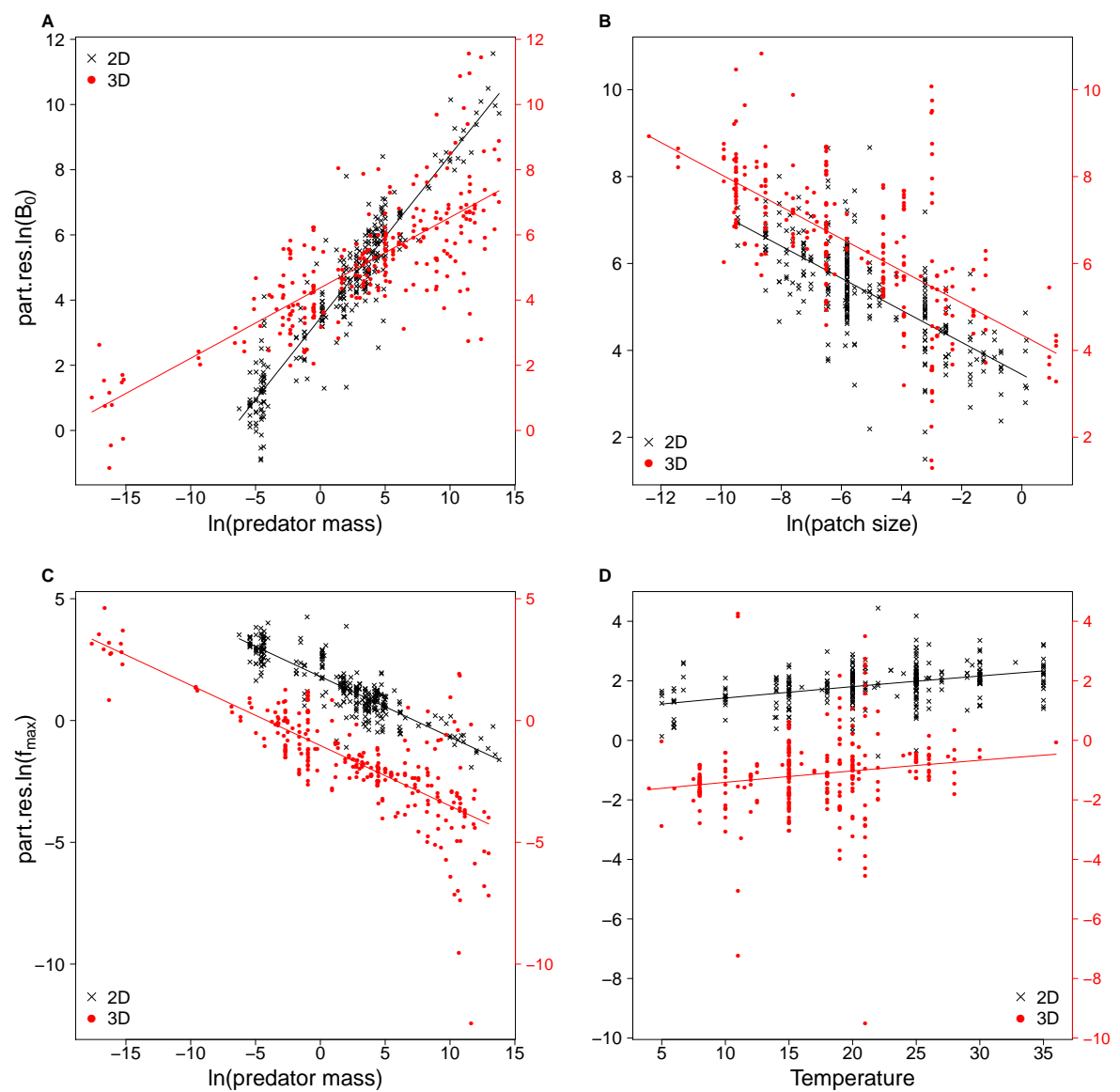

Fig. 4.3: Statistical results of the dependencies of functional response parameters using the dataset. All the variables other than temperature are expressed as $\ln$-transformed and partial residuals plots are presented (details of partial residuals plots in the supporting information). Panels A and B show the results for half-saturation densities $\left(B_{0}\left[\mathrm{mg} \mathrm{m}^{-2} \mid \mathrm{mg} \mathrm{m}^{-3}\right]\right)$ and their scalings with predator mass and patch size, respectively. Panels $C$ and $D$ show the results for specific maximum feeding rates $\left(f_{\max }\left[\mathrm{mg} \mathrm{d}^{-1} \mathrm{mg}^{-1}\right]\right)$ and their scalings with predator mass and environmental temperature, respectively. Data points for different dimensional scenarios (2D, 3D) are plotted with different colors and styles (see legend).

\section{Discussion}

Our study quantified the effects of global warming and habitat loss on feeding interaction strengths (i.e. functional response parameters) by a meta-analysis and consequently illustrated the combined effects of warming and habitat loss on simulated food chains by integrating feeding-related and other biological rates. We showed that global warming increases the energy intake of predators (through increasing the specific maximum feeding rates of predators in feeding interactions) and habitat loss decreases the feeding efficiencies of predators (by decreasing the half-saturation densities). Therefore the combined effects of warming and habitat loss severely damage the food chains, causing consumers to go extinct. 


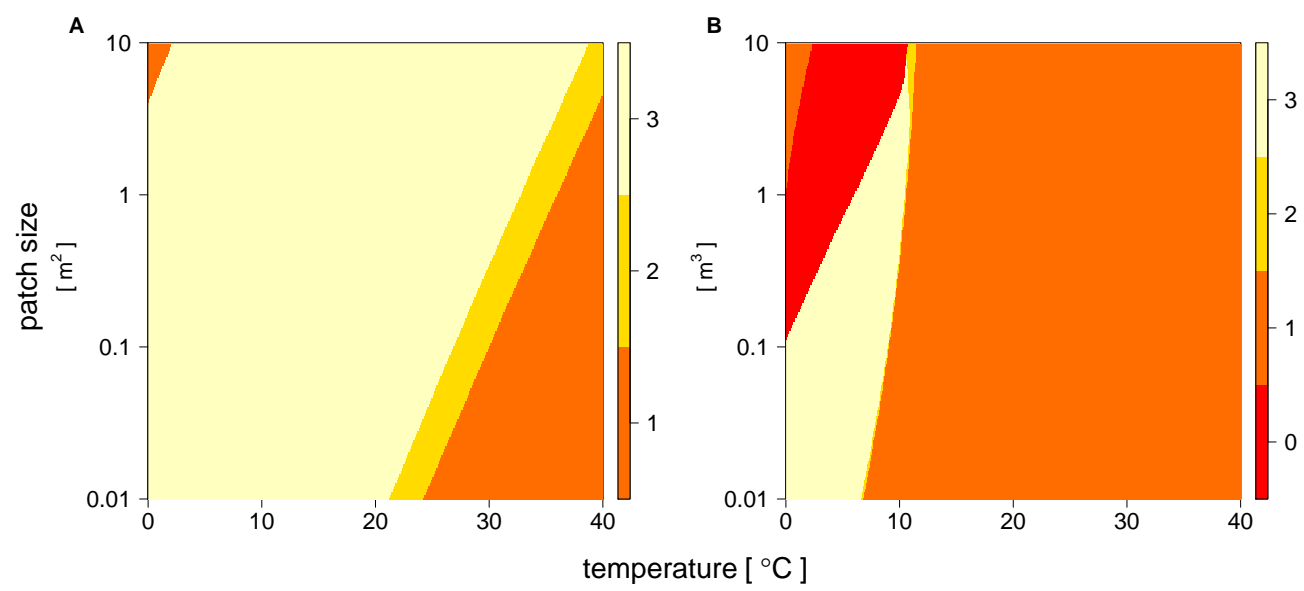

Fig. 4.4: Number of surviving species (see color codes) for the food-chain models after 274 years (100 000 steps). Different dimensional cases are shown in panel A (2D) and panel B (3D). Patch size (y-axis) plotted are transformed by common logarithm $\left(\log _{10}\right)$, but the actual patch-size values are shown.

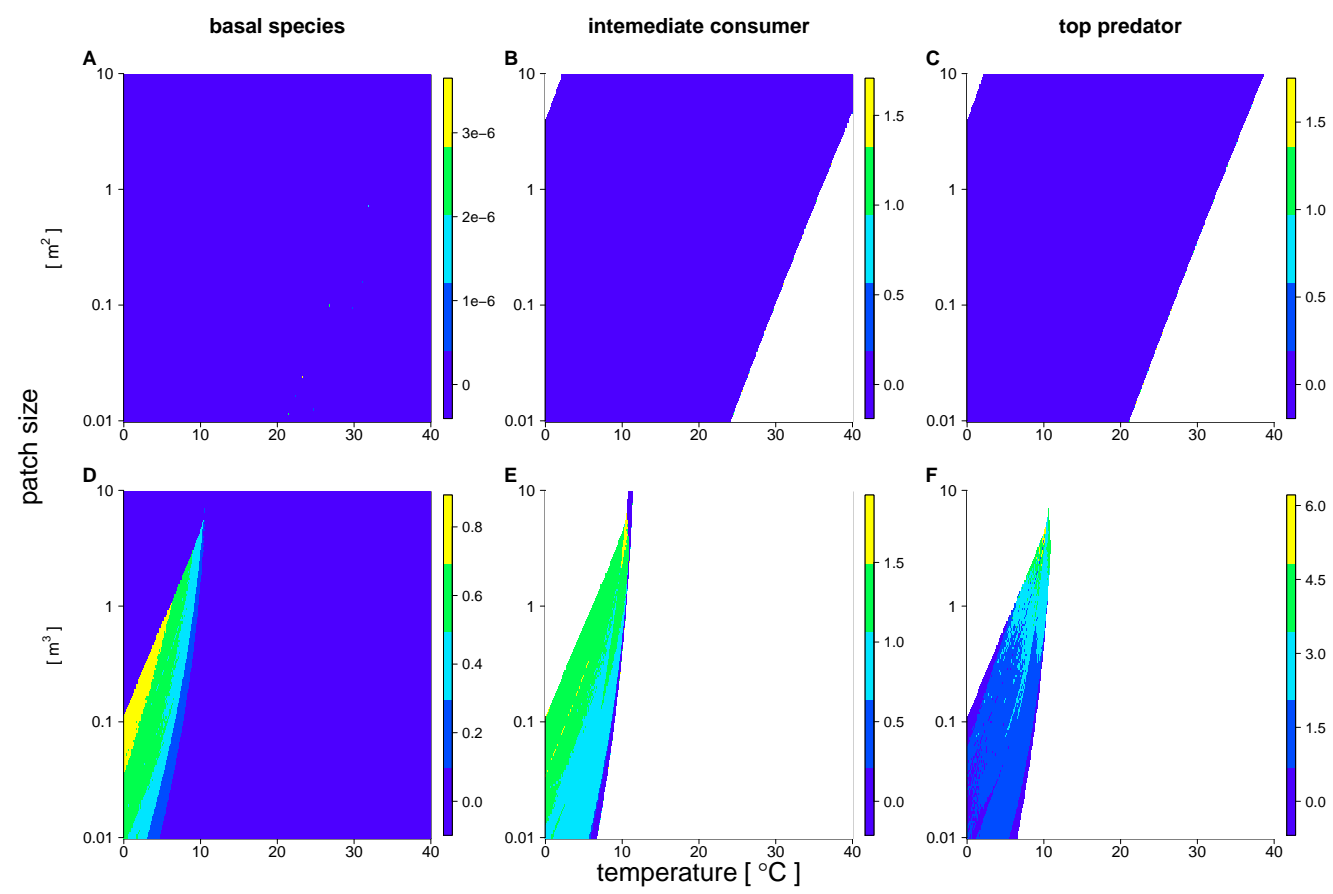

Fig. 4.5: Coefficient of variation (CV) for the surviving species in the food chain models (see color code). The CVs are calculated by the last 10000 steps (27.4 years) of simulations. The CVs for basal species (panels A and D), intermediate consumers (panels $\mathrm{B}$ and $\mathrm{E}$ ) and top predators (panels $\mathrm{C}$ and $\mathrm{F}$ ) are shown in different columns. The panels in the upper row are for the two-dimensional cases and the ones in the lower row for three-dimensional cases. Patch size (y-axis) plotted are transformed by common logarithm $\left(\log _{10}\right)$, but the values for actual patch sizes are shown.

Specific maximum feeding rates decrease with predator mass by a power-law scaling of -0.248 , which is remarkably consistent with the theoretical prediction, $-\frac{1}{4}$ (Brown 
et al., 2004). As the metabolic theory predicts that the specific metabolic rate scales with body mass by an exponent $-\frac{1}{4}$, the energy gain from feeding shall also (at least) have the same scale, which is widely applied in food web studies. Habitat loss does not influence the specific maximum feeding rate. This result confirms our hypothesis that this rate is only physiologically related, as the metabolic theory predicted that maximum feeding rate only depend on the time components of ingesting and digesting the prey (Brown et al., 2004; Brose, 2010). This assumption is also supported by the fact that there is no scaling difference between $2 \mathrm{D}$ and $3 \mathrm{D}$. Nevertheless, Pawar et al. (2012) separated 2D and 3D when analyzing handling time (reverse of maximum feeding rate) as they argued that different dimensionalities imposed different physical constraints on consumers. This is probably due to their suggestion that the time needed to pursue potential prey shall be included in the definition of handling time. We argue that the time for pursuing should influence attack rate, as handling time, $h$ only counts for the time spend on successfully attacked prey items, but a predator could try to pursue a prey and fail. Researchers can have different assumptions on functional response components even with the same model (Okuyama, 2012) which is an important point to be aware of when comparing results in different studies.

Our results of the meta-analysis showed that the half-saturation density does not scale with temperature which is consistent with Hansen et al. (1997) and confirmed by Rall et al. (2012) and Fussmann et al. (2014) using a similar dataset. However, the half saturation density increases with increasing predator mass and habitat loss. Previous studies often assumed that it did not scale with predator mass (e.g. Brose et al., 2006b) due to the lack of deterministic supports of the scaling of attack rate, $a\left(B_{0}=\frac{1}{a h}\right)$, theoretically (McGill and Mittelbach, 2006) and empirically (Hansen et al., 1997). However, we found an increase of half saturation density with predator mass. Our results also showed that the scalings of half saturation densities are different for two- and three-dimensional spaces. This dimensional difference may rely on the impacts of dimensionality on the movements of predators and prey and their encounter probability, as McGill and Mittelbach (2006) suggested that visual receptions between 2D and 3D dimensional spaces shall be different. Furthermore, as the allometric scaling of half saturation density with predator mass is shallower in three-dimensional spaces, we infer that the encounter rates of predators and prey are lower in a three-dimensional space than in a two-dimensional space.

The result that half-saturation density increases with habitat loss is the key why habitat loss fosters the warming effect on biodiversity loss, consistent with Li et al. (2017a) who also found that decreasing patch size (habitat loss) is likely to increase half-saturation density based on an individual-based simulation modeling. This result confirmed our hypothesis that habitat loss would decrease feeding rates. This results may be because of the different experimental setups between micro-organism and large predators in the dataset. For micro-organisms, their feeding trails must 
involve a group of predator individuals (e.g. Wickham, 1995) whereas for other larger predator, the feeding trails are typically carried out for only one predator individuals (e.g. Spitze, 1985). Therefore, for smaller predators which are also associated with smaller patch sizes, the intra-specific competition may contribute to a higher half-saturation density. Nevertheless, predator mass and patch size are not considerably correlated (Person's correlation being 0.67), Fig. S4.2 in the supporting information. This suggested that the mentioned caveat above shall only be of minor importance for our results. Other reasons can be related to behavioral changes that species got less active and caught less prey in smaller patches (Bergström and Englund, 2004) and the edge effect that in smaller patches, individuals tend to be stuck at the edges (Li et al., 2017a). However, these behavioral changes still have their conversational meanings, as the current human-induced habitat loss is often associated with habitat fragmentation and degradation (Fahrig, 1997). We suggested that future studies aiming to investigate feeding interactions should be aware of the importance to choose an appropriate experimental arena which allows their results to be applied to natural ecosystems.

According to the food chain simulations, more species go extinct with increasing temperature in both two- and three-dimensional scenarios and decreasing patch size fosters this negative effect on consumer species. With increasing temperature, the metabolic demands of the consumers increase more than their abilities of obtaining energy (i.e. feeding rates). Therefore, consumers and especially the species in higher trophic levels are first affected and may go extinct due to starvation (Binzer et al., 2012). In cases of warming and habitat loss, not only the metabolic demands of consumers increase (due to warming), but the feeding efficiencies also decrease due to the effect of habitat loss. Therefore, consumer species are more vulnerable than under the isolated influence of warming. However, species coexistence in different ecosystems under the influences of multiple global change drivers show complex patterns. For example, when temperature is low, increasing patch size in three-dimensional cases also results in species loss (Fig. 4.4B). The different consequences of enlargement of patch size at lower or higher temperatures are due to different mechanisms. When temperature is low and patch size is large, the biomass density of basal species is limited as the growth of basal species is low due to low temperature, whereas, consumers are very efficient as half-saturation density decreases with increasing habitat size. In this case, consumers will starve to death which refers to as bottom-up effect (see plot of timeseries, Fig. S4.3 in the supporting information). Yet, in case of a low temperature and a small patch, the bottom-up effect is relaxed as the feeding efficiency of consumers are low due to the effect of habitat loss on half-saturation density. Therefore, all the species survived but with highly dynamic population-cycles (see Fig. 4.5 and Fig. S4.4 in the supporting information). 
Up to the food web level, previous studies (e.g. Petchey et al., 1999; Post et al., 2000; Binzer et al., 2016) indicated the negative effect of either warming or habitat loss. Inferring from our results, we expect that the single and combined effects of warming and habitat loss first affect higher trophic level species and further result in the loss of entire higher trophic levels. Our meta-analysis showed that warming decreases relative energy flux to energy loss of consumers, which is consistent with Binzer et al. (2012); Fussmann et al. (2014). Our results also indicated an increase in half-saturation density with habitat loss. Under the reduced population sizes of intermediate-level consumers due to warming, habitat loss further fosters the strength of deceased energy flux relative to energy loss. Therefore, in a structured food web (consumers have larger body masses than their resources), we expect biodiversity loss and loss of trophic levels in the combined effects of warming and habitat loss.

Our simulations on simple food chains suggest that, in general, the range of the combinations of temperature and patch size which supported all three species in three-dimensional space is smaller than in two-dimensional space. This may due to the lower assimilation coefficient for the intermediate consumer, lower growth rate of the basal species (plant) and the larger predator-prey body-mass ratio in three-dimensional cases than in two-dimensional cases (Eq. (4.6)). This result is in contrast to Pawar et al. (2012) that they found advantages for consumers in three-dimensional environments (as it supported large population sizes and a larger range of predator-prey pairs). This is probably due to the fundamental differences of the body-mass scaling of half-saturation density. In the population dynamics model of Pawar et al., half-saturation densities (calculated to be comparable with ours) scale with consumer mass by a scaling of 0.07 and -0.31 in $2 \mathrm{D}$ and $3 \mathrm{D}$ but 0.50 and 0.217 in ours. More steeply increasing half-saturation densities is an important reason why $3 \mathrm{D}$ has smaller range to support a food chain in our model. Given decreasing half-saturation densities with body mass in the 3D scenario of Pawar et al. (2012), much higher baseline constant (comparable $a_{K}$ in our model) is probably another necessary condition for their model to obtain the advantage of 3D.

The most important result of our study is that habitat loss fosters the effect of warming on biodiversity loss, which is consistent with the empirical studies as summarized by Mantyka-Pringle et al. (2012). Furthermore, it is ecologically important to study the combined effects of climate warming and habitat loss (Brook et al., 2008). Land use change is one of the most influential human impact on ecosystem. It would directly result in habitat loss and fragmentation, which further may foster local warming Smith et al. (2014).

Thus, researchers attempted to understand how global environmental changes (GEC) (e.g. global warming and habitat loss) affect biodiversity and ecosystem by metadata analysis (e.g. Tylianakis et al., 2008) or snapshots of controlled experiments 
(e.g. Dossena et al., 2012; Shurin et al., 2012). As species in an ecosystem interact with each other under the context of a network, the interactions of a specific pair of species are affected by all the other interactions involving those species. Therefore, it's difficult to understand how one or several global environmental factors influence a specific interaction without considering it as a part of the ecological network. Similarly, as the ecological networks are universally dynamic, only one or several snapshots of a system (e.g. Dossena et al., 2012; Shurin et al., 2012) is not enough to show the general response of this ecosystem to some GEC drivers. If conditions allow, time-series data would be better to show and understand the response and stability of an ecosystem (e.g. Fussmann et al., 2014). To understand the mechanisms, we need to design specific research questions to be able to measure how global change drivers affect basic biological rates. From the point of view of interaction strengths, the mechanism of how attack rates are influenced by body mass in different dimensions also need a lot more effort. 
Part III

General discussion 



\section{Discussion}

\section{Habitat degradation associated with warm- ing harms biodiversity}

This thesis explores how land-use change, with the association of climate change, influences species' feeding interactions and the resulting consequences to biodiversity. The influences of land-use change are represented by habitat degradations (habitat loss and habitat simplification) and climate change by warming. Feeding interaction strengths are modeled by Holling's type II or type III functional responses and I tried to quantify how functional response parameters scale with habitat loss and simplification. Their relevance to biodiversity are demonstrated by a predator-prey system and a tritrophic food chain. Due to the potentially inaccurate estimations of interaction strengths by results of individual feeding studies, I first investigated the effects of experimental duration and predator satiation level on the estimates of functional response parameters by a meta-analysis in chapter 2 . This offers a better understanding of feeding processes and how to improve the estimates of interaction strengths. I, then, studied the effects of habitat loss and habitat simplification on interaction strengths and a predator-prey system by a simulation model (IBM) and an analytically-solved model (ODE), respectively (chapter 3). Finally, I combined the meta-analytical approach and modeling approach to illustrate the synergistic effect of habitat loss and global warming on interaction strengths and persistence of food chains (chapter 4). The results showed that both habitat loss and simplification severely influenced feeding interaction strengths, leading to a loss in biodiversity.

There exist a potential barrier between individual feeding experiments and population dynamic models in food web studies. If functional response parameters estimated from individual feeding studies serve as a good estimation of interaction strengths from a viewpoint of long-term population dynamics remained largely untested. To improve such estimation, my results in chapter 2 pointed out the importance of experimental duration and predator satiation level. The attack rate decreases with increasing experimental durations. The handling time is larger for satiated predators than for starved ones. Therefore, these results have important implications. They suggest to carefully choose a relatively longer period of time, during when the feeding processes are more representative for the life cycle of the predator. They also suggest that theorists could find a better conversion for the inappropriate estimates of interaction strengths, rather than use them directly. As the satiation level of predator also affects handling time, the results of this study 
suggest the importance to average the effect of predator satiation, e.g. by using predators with half-full guts or report the pre-feeding treatments to predators more carefully.

The range of experimental durations covered in this chapter was mostly within 24 hours. Therefore, I explained the rationale of the influence of experimental duration from two major processes in a diurnal cycle, feeding and resting. The longer a feeding experiment, the more likely that resting will be involved which would strongly influence the attack rate. The effect of predator satiation level can be interpreted by ,digestive limit' which targets on the joint influences of gut size and digestion rate (Jeschke, 2007). If a predator can fill up its gut faster than digestion, the predator will then face a digestive limit. The result that handling times for satiated predators are larger than for starved predators is a reflection for the rationality of this explanation. Yet the data I analyzed in this chapter are mostly from laboratory studies. Whether consumers in the field are also facing ,digestive limits' is open to be investigated. One field study did test this question and suggested that a fish predator (Micropterus salmoides showed digestive limit in the field (Essington et al., 2000)). Even though the dataset I analyzed in this research chapter is mostly comprised of the feeding studies in the laboratory, they have a strong connection with interaction strengths used in theoretical studies of food webs. Whereas, feeding studies in the field are mostly studied with a focus on conservation (for terrestrial carnivores) or for fishery production, where they quantify the functional responses by quite different approaches (Hunsicker et al., 2011; Zimmermann et al., 2015).

The laborious nature of functional response experiments and the limits of empirical experiments (e.g. sizes of experimental arenas and uncontrollable side effects of adding habitat complexities) both hinder the development of spatial ecology in the field of feeding interactions. Therefore, I constructed an individual-based model (IBM) to carry out in silico feeding experiments. It allowed me to investigate a large ingredient of patch sizes (from experimental arena to field plot) and disentangle the complex effects of habitat complexity. I also constructed a predator-prey model with ordinary differential equations (ODE) in light of these in silico feeding experiments. The results in chapter 3 showed that half saturation density increased with increasing habitat complexity (represented by refuge availability for prey). Yet, patch size did not influence any functional response parameters. The increasing half saturation density indicated that the feeding efficiency of the predator decreased. With reduced feeding efficiencies, the predator-prey system became more viable. The reason why decreasing feeding efficiencies increase the viability of the predator-prey system is that it decreases the energy gain relative to energy loss for the predator which would lead to more stable population sizes of both predator and prey (Rip and McCann (2011), more details below). Yet, I suspected the reason why patch size did not affect functional response parameters (statistically not significant) might lie in the 
fact that I only constructed a simple IBM without more behavioral complexities (see Bergström and Englund, 2004, for the case with more behaviors).

Bearing in mind that in chapter 3 , the question remained whether habitat loss had an effect on functional response parameters, I conducted a meta-analysis and constructed a tritrophic model of food chain. They allowed to investigate the effects of habitat loss and warming on feeding interaction strengths and therefore biodiversity. The results of this study (chapter 4) suggested, for the first time, that the half saturation density decreased with increasing patch size based on a dataset containing data systematically across different taxa and ecosystems. The results also confirmed that the maximum feeding rate increases with temperature (Rall et al., 2012; Fussmann et al., 2014). Combining the general rules on the scalings of other major physiological rates (i.e. growth rate of basal species, carrying capacity of environment and metabolic rate) with body mass and temperature, I showed by a tritrophic model of food chain that the synergistic effect of habitat loss and warming harmed the persistence of food chains. The result of reduced half saturation density with increasing patch size is a key finding in this thesis. Even though I found a study of video tracking supporting this finding, there exist much more to be investigated in more details in the future (e.g. the mechanistic explanations). This study of video tracking observed a behavioral change of species when patches were getting larger (Bergström and Englund, 2004). They found that species got more active and caught more prey in larger patches. However, due to time restraints, I was not able to check if there was intra-specific competition of predators in the studies involved in this database. If competition among predators plays a role, it may contribute to the effect of patch size on half saturation density. Studies in the future shall investigate this point to better understand the mechanism behind the effect of patch size on functional response parameters.

I managed to identify the dimensionality of the data used in the meta-analysis of chapter 4. I also parameterized the food-chain model in this chapter according to different dimensionalities. The results of these two dimensional cases showed consistency in major aspects. However, species persistence in food chains when temperature is low and patch size is large showed quite different patterns in twoand three-dimensional cases. In three-dimensional case when temperature is low, the surviving species decreased with increasing patch size, which did not corroborate my general findings from the meta-analysis. This is because of the reduced surviving species here is induced by another mechanism. The reduced surviving species in three-dimensional space is driven by the large variability of population densities. Whereas, in the corresponding two-dimensional space, the populations were much more stable. Rip and McCann (2011) suggested that any causes which lead to an energy gain relative to energy loss would push the multi-population systems towards oscillation. The relative energy decrease with increasing habitat complexity did make the predator-prey system more viable and stable (chapter 3). Yet the relative 
energy decrease with decreasing patch size (i.e. habitat loss), within the range I investigated in chapter 4 , in general decrease the supporting number of species in food chains. This is mainly due to the large range of patch sizes that I investigated. The degree of energy decrease with habitat loss has been large enough to turn the population densities to zero. But within the narrow domain where all three species survived in three-dimensional cases, the population dynamics indeed get more stable with habitat loss (see the coefficient of variation plots in Fig. 4.5).

Separating different dimensional cases has other major benefits. Habitat loss may harm different dimensional ecosystems differently, e.g. ocean systems are dominated by three-dimensional interactions and terrestrial ecosystems by two-dimensional interactions (Barrios-O'Neill et al., 2016). Furthermore, to appropriately quantify the functional response parameters, it is necessary to disentangle data from different dimensions. This is especially necessary as the direct interaction between two individuals (i.e. searching, hiding, moving) operates differently in two- and three-dimensional environments (McGill and Mittelbach, 2006) and the half saturation density is directly dependent on the dimensionality (i.e. biomass per area in two-dimensional patches and biomass per volume in three-dimensional patches). Consequently, half saturation densities in different dimensionalities might respond differently to habitat loss. This is a point that future studies should be dedicated to investigate.

Even though I did not manage to include extinction boundaries for populations in the food-chain model of chapter 4 as I did in the predator-prey model of chapter 3 , this point should be included in the future studies. The idea behind extinction boundaries is among one of the oldest considerations in spatial ecology, i.e. ecosystem-size hypothesis (Holt, 1993): a decrease of patch size decreases the absolute amount of available resources in the whole patch and therewith leads to an energy limitation that threatens most likely the upper trophic levels. Incorporating such extinction boundaries (e.g. two individuals per patch) into population-based models would allow a better implementation of space into of traditional food web studies (Kalinkat et al., 2015). It can be done either by modifying the extinction boundaries according to this individual concept in the algorithm of ODE simulations or to compare the ending population densities from these ODE simulations with these individual-based extinction boundaries afterwards. Yet future studies should be dedicated to figure out and test for better techniques to incorporate this.

Some researchers suspected that the environmental carrying capacity (i.e. supporting biomass per unit of size) would decrease with habitat loss, i.e. productivity-space hypothesis (Elton, 1927). Since such research is difficult to carry out, I was not able to include this point in the simulation models due to lack of empirical support (chapter 3 and 4). I parameterized the constants of carrying capacities (i.e. $a_{K}$ ) in different dimensional spaces in chapter 4 according to different empirical studies, in 
order to account for some influence from spatial properties. Future studies should focus on such issues to gain a better understanding of the influences of space.

Allometric scalings were not only used to parameterize the variables in ODEs for population dynamics models (i.e. major physiological rates which decide energy flux in chapter 3 and 4), but also to parameterize the behaviors involved in feeding processes (i.e. attack success, digestion rate and gut size in chapter 3 ). The metabolic theory of ecology is capable of incorporating these behaviors as they are also limited by the physiological and chemical properties of molecular and cellular components for life (Brown et al., 2004). Yet, due to the lack of empirical support, most of the feeding-related parameters (hereby digestion-related) in the IBM model were parameterized based on data from one single study combined with rational guess. A more mechanistically understanding of the behaviors involved in feeding processes could be elaborated in the future studies.

The food-chain models I used in chapter 4 nicely demonstrate and explain how the ensemble of species interaction (hereby feeding) and other major physiological rates integrally determine the survival of species and they are also capable of inferring the basic pattern of food webs, e.g. higher trophic level species are more vulnerable to habitat loss and warming (Dobson et al., 2006; Shurin et al., 2012). Yet they are not able to show more detailed behavior of some species in a food web, such as omnivores. Especially the omnivores who feed on both resource and other consumer species, as habitat loss does not directly influence the major physiological rate of resource species, i.e. intrinsic growth rate yet does influence the feeding efficiency of consumer species. In order to understand the mechanism of habitat modification on biodiversity from the viewpoint of species interaction and other major physiological processes, future studies can go beyond the feeding interaction which is the main focus in this thesis to investigate other important physiological processes.

Altogether, this thesis provides a systematic investigation of the influences of habitat modifications on biodiversity utilizing two modeling tools, individual-based modeling and population-based modeling of ODEs, combined with a meta-analysis. The results not only confirmed the importance of habitat complexity on supporting biodiversity but also suggested the influence of habitat loss on biodiversity loss and its aggravating effect on fostering global warming to induce biodiversity loss. I also utilized allometric scalings to parameterize feeding-related behaviors, providing a model framework which was capable of testing a lot of potential important mechanisms involved in feeding-related studies. For example, by controlling the satiation level of the predator, it showed the direct effect of predator satiation on feeding interaction strengths (Scotti et al., 2017). In the ODE model of food-chain, I was able to include absolute patch sizes by combing a set of empirically supported allometric scalings. This improvement would aid to better explore research questions of spatial ecology in food webs as the models before used to be normalized to only have relative space 
and time (e.g. Otto et al., 2007). The approach in this thesis of focusing on the effects of global change on individual physiological rates and integrate these parts which control energy flux into ecological networks allows to better investigate the mechanisms of the influence of global change on biodiversity. I therefore address the importance of species interactions and other major physiological traits on the research of biodiversity and global change. 
Appendix 



\section{Bibliography}

Aljetlawi, A. A., Sparrevik, E., and Leonardsson, K. (2004). Prey-predator sizedependent functional response: derivation and rescaling to the real world. Journal of Animal Ecology, 73(2):239-252.

Anderson, R. M., Whitfield, P. J., Dobson, A. P., and Keymer, A. E. (1978). Concomitant predation and infection processes: an experimental study. Journal of Animal Ecology, 47(3):891-911.

Attard, P. (2006). Non-periodic boundary conditions for molecular simulations of condensed matter. Molecular Physics, 104(12):1951-1960.

Barrios-O’Neill, D., Kelly, R., Dick, J. T. A., Ricciardi, A., MacIsaac, H. J., and Emmerson, M. C. (2016). On the context-dependent scaling of consumer feeding rates. Ecology Letters, 19(6):668-678.

Bartoń, K. (2016). MuMIn: Multi-Model Inference. R package version 1.15.6.

Bergström, U. and Englund, G. (2004). Spatial scale, heterogeneity and functional responses. Journal of Animal Ecology, 73(3):487-493.

Berlow, E. L., Neutel, A.-M., Cohen, J. E., de Ruiter, P. C., Ebenman, B., Emmerson, M., Fox, J. W., Jansen, V. A. A., Iwan Jones, J., Kokkoris, G. D., Logofet, D. O., McKane, A. J., Montoya, J. M., and Petchey, O. (2004). Interaction strengths in food webs: issues and opportunities. Journal of Animal Ecology, 73(3):585-598.

Binzer, A., Guill, C., Brose, U., and Rall, B. C. (2012). The dynamics of food chains under climate change and nutrient enrichment. Phil. Trans. R. Soc. B, 367(1605):2935-2944.

Binzer, A., Guill, C., Rall, B. C., and Brose, U. (2016). Interactive effects of warming, eutrophication and size structure: impacts on biodiversity and food-web structure. Global Change Biology, 22(1):220-227.

Boit, A., Martinez, N. D., Williams, R. J., and Gaedke, U. (2012). Mechanistic theory and modelling of complex food-web dynamics in Lake Constance. Ecology Letters, 15(6):594-602. 
Bolker, B. and R Development Core Team (2014). bbmle: Tools for general maximum likelihood estimation. R package version 1.0.17.

Bolker, B. M. (2008). Ecological models and data in R. Princeton Univ. Press, Princeton, NJ.

Bowler, D. E., Hof, C., Haase, P., Kröncke, I., Schweiger, O., Adrian, R., Baert, L., Bauer, H.-G., Blick, T., Brooker, R. W., Dekoninck, W., Domisch, S., Eckmann, R., Hendrickx, F., Hickler, T., Klotz, S., Kraberg, A., Kühn, I., Matesanz, S., Meschede, A., Neumann, H., O’Hara, R., Russell, D. J., Sell, A. F., Sonnewald, M., Stoll, S., Sundermann, A., Tackenberg, O., Türkay, M., Valladares, F., van Herk, K., van Klink, R., Vermeulen, R., Voigtländer, K., Wagner, R., Welk, E., Wiemers, M., Wiltshire, K. H., and Böhning-Gaese, K. (2017). Cross-realm assessment of climate change impacts on species' abundance trends. Nature Ecology \& Evolution, 1(3):0067.

Brook, B. W., Sodhi, N. S., and Bradshaw, C. J. A. (2008). Synergies among extinction drivers under global change. Trends in Ecology \& Evolution, 23(8):453-460.

Brose, U. (2010). Body-mass constraints on foraging behaviour determine population and food-web dynamics. Functional Ecology, 24(1):28-34.

Brose, U., Blanchard, J. L., Eklöf, A., Galiana, N., Hartvig, M., R. Hirt, M., Kalinkat, G., Nordström, M. C., O’Gorman, E. J., Rall, B. C., Schneider, F. D., Thébault, E., and Jacob, U. (2016). Predicting the consequences of species loss using size-structured biodiversity approaches. Biological Reviews, pages 1-14.

Brose, U., Ehnes, R. B., Rall, B. C., Vucic-Pestic, O., Berlow, E. L., and Scheu, S. (2008). Foraging theory predicts predator-prey energy fluxes. Journal of Animal Ecology, 77(5):1072-1078.

Brose, U., Jonsson, T., Berlow, E. L., Warren, P., Banasek-Richter, C., Bersier, L.-F., Blanchard, J. L., Brey, T., Carpenter, S. R., Blandenier, M.-F. C., Cushing, L., Dawah, H. A., Dell, T., Edwards, F., Harper-Smith, S., Jacob, U., Ledger, M. E., Martinez, N. D., Memmott, J., Mintenbeck, K., Pinnegar, J. K., Rall, B. C., Rayner, T. S., Reuman, D. C., Ruess, L., Ulrich, W., Williams, R. J., Woodward, G., and Cohen, J. E. (2006a). Consumer-resource body-size relationships in natural food webs. Ecology, 87(10):2411-2417.

Brose, U., Williams, R. J., and Martinez, N. D. (2006b). Allometric scaling enhances stability in complex food webs. Ecology Letters, 9(11):1228-1236.

Brown, J. H., Gillooly, J. F., Allen, A. P., Savage, V. M., and West, G. B. (2004). Toward a metabolic theory of ecology. Ecology, 85(7):1771-1789. 
Buckel, J. A. and Stoner, A. W. (2000). Functional response and switching behavior of young-of-the-year piscivorous bluefish. Journal of Experimental Marine Biology and Ecology, 245(1):25-41.

Bundy, A. (2004). Mass balance models of the eastern Scotian Shelf before and after the cod collapse and other ecosystem changes. Can. Tech. Rep. Fish. Aquat. Sci., 2520:xii + 193

Casas, J. and McCauley, E. (2012). Daily foraging cycles create overlapping timescales in functional responses. Oikos, 121(12):1966-1976.

Crawley, M. J. (2007). The R book. Wiley, Chichester, England; Hoboken, N.J. OCLC: 163190367.

Dale, B. W., Adams, L. G., and Bowyer, R. T. (1994). Functional response of wolves preying on barren-ground caribou in a multiple-prey ecosystem. Journal of Animal Ecology, 63(3):644-652.

de Ruiter, P. C., Neutel, A.-M., and Moore, J. C. (1995). Energetics, patterns of interaction strengths, and stability in real ecosystems. Science, 269(5228):12571260 .

Dobson, A., Lodge, D., Alder, J., Cumming, G. S., Keymer, J., McGlade, J., Mooney, H., Rusak, J. A., Sala, O., Wolters, V., Wall, D., Winfree, R., and Xenopoulos, M. A. (2006). Habitat loss, trophic collapse, and the decline of ecosystem services. Ecology, 87(8):1915-1924.

Dossena, M., Yvon-Durocher, G., Grey, J., Montoya, J. M., Perkins, D. M., Trimmer, M., and Woodward, G. (2012). Warming alters community size structure and ecosystem functioning. Proceedings of the Royal Society of London B: Biological Sciences, 279(1740):3011-3019.

Ebeling, A. W. and Bray, R. N. (1976). Day versus night activity of reef fishes in a kelp forest off Santa Barbara, California. Fishery Bulletin, 74(4):703-717.

Ehnes, R. B., Rall, B. C., and Brose, U. (2011). Phylogenetic grouping, curvature and metabolic scaling in terrestrial invertebrates. Ecology Letters, 14(10):993-1000.

Elton, C. S. (1927). Animal ecology. Macmillan Company, New York.

Essington, T. E., Hodgson, J. R., and Kitchell, J. F. (2000). Role of satiation in the functional response of a piscivore, largemouth bass (Micropterus salmoides). Canadian Journal of Fisheries and Aquatic Sciences, 57(3):548-556.

Fahrig, L. (1997). Relative effects of habitat loss and fragmentation on population extinction. The Journal of Wildlife Management, 61(3):603-610. 
Fahrig, L. (2003). Effects of habitat fragmentation on biodiversity. Annual Review of Ecology, Evolution, and Systematics, 34(1):487-515.

Folsom, T. C. and Collins, N. C. (1984). The diet and foraging behavior of the larval dragonfly Anax junius (Aeshnidae), with an assessment of the role of refuges and prey activity. Oikos, 42(1):105-113.

Fox, L. R. and Murdoch, W. W. (1978). Effects of feeding history on short-term and long-term functional responses in Notonecta hoffmanni. Journal of Animal Ecology, 47(3):945-959.

Fussmann, K. E., Schwarzmüller, F., Brose, U., Jousset, A., and Rall, B. C. (2014). Ecological stability in response to warming. Nature Climate Change, 4(3):206-210.

Galarowicz, T. L. and Wahl, D. H. (2005). Foraging by a young-of-the-year piscivore: the role of predator size, prey type, and density. Canadian Journal of Fisheries and Aquatic Sciences, 62(10):2330-2342.

Gergs, A. (2011). Modelling foraging behaviour in the insect predator Notonecta maculata using the individuals approach. PhD thesis, RWTH Aachen University.

Gergs, A. and Ratte, H. T. (2009). Predicting functional response and size selectivity of juvenile Notonecta maculata foraging on Daphnia magna. Ecological Modelling, 220(23):3331-3341.

Gibson-Reinemer, D. K., Sheldon, K. S., and Rahel, F. J. (2015). Climate change creates rapid species turnover in montane communities. Ecology and Evolution, 5(12):2340-2347.

Gillooly, J. F., Brown, J. H., West, G. B., Savage, V. M., and Charnov, E. L. (2001). Effects of size and temperature on metabolic rate. Science, 293(5538):2248-2251.

Gompertz, B. (1825). On the nature of the function expressive of the law of human mortality, and on a new mode of determining the value of life contingencies. Philosophical Transactions of the Royal Society of London, 115(0):513-583.

Gotoh, T., Nozawa, M., and Yamaguchi, K. (2004). Prey consumption and functional response of three acarophagous species to eggs of the two-spotted spider mite in the laboratory. Applied Entomology and Zoology, 39(1):97-105.

Gray, C., Baird, D. J., Baumgartner, S., Jacob, U., Jenkins, G. B., O'Gorman, E. J., Lu, X., Ma, A., Pocock, M. J. O., Schuwirth, N., Thompson, M., and Woodward, G. (2014). Ecological networks: the missing links in biomonitoring science. Journal of Applied Ecology, 51(5):1444-1449.

Gregorini, P., Tamminga, S., and Gunter, S. A. (2006). Behavior and daily grazing patterns of cattle. The Professional Animal Scientist, 22(3):201-209. 
Grimm, V., Berger, U., Bastiansen, F., Eliassen, S., Ginot, V., Giske, J., Goss-Custard, J., Grand, T., Heinz, S. K., Huse, G., Huth, A., Jepsen, J. U., Jørgensen, C., Mooij, W. M., Müller, B., Pe'er, G., Piou, C., Railsback, S. F., Robbins, A. M., Robbins, M. M., Rossmanith, E., Rüger, N., Strand, E., Souissi, S., Stillman, R. A., Vabø, R., Visser, U., and DeAngelis, D. L. (2006). A standard protocol for describing individual-based and agent-based models. Ecological Modelling, 198(1-2):115126.

Grimm, V., Berger, U., DeAngelis, D. L., Polhill, J. G., Giske, J., and Railsback, S. F. (2010). The ODD protocol: A review and first update. Ecological Modelling, 221(23):2760-2768.

Hansen, P. J., Bjørnsen, P. K., and Hansen, B. W. (1997). Zooplankton grazing and growth: Scaling within the $2-2000-\mu \mathrm{m}$ body size range. Limnology and Oceanography, 42(4):687-704.

Harvey, E., Gounand, I., Ward, C. L., and Altermatt, F. (2016). Bridging ecology and conservation: from ecological networks to ecosystem function. Journal of Applied Ecology.

Hauzy, C., Tully, T., Spataro, T., Paul, G., and Arditi, R. (2010). Spatial heterogeneity and functional response: an experiment in microcosms with varying obstacle densities. Oecologia, 163(3):625-636.

Herbers, J. M. (1981). Time resources and laziness in animals. Oecologia, 49(2):252262.

Hoddle, M. S. (2003). The effect of prey species and environmental complexity on the functional response of Franklinothrips orizabensis: a test of the fractal foraging model. Ecological Entomology, 28(3):309-318.

Hohberg, K. and Traunspurger, W. (2005). Predator-prey interaction in soil food web: functional response, size-dependent foraging efficiency, and the influence of soil texture. Biology and Fertility of Soils, 41(6):419-427.

Holling, C. S. (1959a). The components of predation as revealed by a study of small-mammal predation of the European pine sawfly. The Canadian Entomologist, 91(5):293-320.

Holling, C. S. (1959b). Some characteristics of simple types of predation and parasitism. The Canadian Entomologist, 91(7):385-398.

Holling, C. S. (1966). The functional response of invertebrate predators to prey density. Memoirs of the Entomological Society of Canada, 98(48):1-86. 
Holt, R. D. (1993). Ecology at the mesoscale: the influence of regional processes on local communities. In Ricklefs, R. and Schluter, D., editors, Species Diversity in Ecological Communities, pages 77-88. Univ. of Chicago Press, Chicago.

Holt, R. D., Lawton, J. H., Polis, G. A., and Martinez, N. D. (1999). Trophic rank and the species-area relationship. Ecology, 80(5):1495-1504.

Hunsicker, M. E., Ciannelli, L., Bailey, K. M., Buckel, J. A., Wilson White, J., Link, J. S., Essington, T. E., Gaichas, S., Anderson, T. W., Brodeur, R. D., Chan, K.-S., Chen, K., Englund, G., Frank, K. T., Freitas, V., Hixon, M. A., Hurst, T., Johnson, D. W., Kitchell, J. F., Reese, D., Rose, G. A., Sjodin, H., Sydeman, W. J., van der Veer, H. W., Vollset, K., and Zador, S. (2011). Functional responses and scaling in predator-prey interactions of marine fishes: contemporary issues and emerging concepts. Ecology Letters, 14(12):1288-1299.

Ibarrola, I., Arambalza, U., Navarro, J. M., Urrutia, M. B., and Navarro, E. (2012). Allometric relationships in feeding and digestion in the Chilean mytilids Mytilus chilensis (Hupé), Choromytilus chorus (Molina) and Aulacomya ater (Molina): a comparative study. Journal of Experimental Marine Biology and Ecology, 426427:18-27.

Ings, T. C., Montoya, J. M., Bascompte, J., Blüthgen, N., Brown, L., Dormann, C. F., Edwards, F., Figueroa, D., Jacob, U., Jones, J. I., Lauridsen, R. B., Ledger, M. E., Lewis, H. M., Olesen, J. M., van Veen, F. F., Warren, P. H., and Woodward, G. (2009). Ecological networks - beyond food webs. Journal of Animal Ecology, 78(1):253-269.

IPCC (2014a). Climate Change 2014: Impacts, Adaptation, and Vulnerability. Part A: Global and Sectoral Aspects. Contribution of Working Group II to the Fifth Assessment Report of the Intergovernmental Panel on Climate Change. Cambridge University Press, Cambridge, United Kingdom and New York, NY, USA.

IPCC (2014b). Summary for policymakers. In Field, C., Barros, V., Dokken, D., Mach, K., Mastrandrea, M., Bilir, T., Chatterjee, M., Ebi, K., Estrada, Y., Genova, R., Girma, B., Kissel, E., Levy, A., MacCracken, S., Mastrandrea, P., and White, L., editors, Climate Change 2014: Impacts, Adaptation, and Vulnerability. Part A: Global and Sectoral Aspects. Contribution of Working Group II to the Fifth Assessment Report of the Intergovernmental Panel on Climate Change, pages 1-32. Cambridge University Press, Cambridge, United Kingdom and New York, NY, USA,.

Jeschke, J. M. (2007). When carnivores are "full and lazy". Oecologia, 152(2):357364.

Jeschke, J. M., Kopp, M., and Tollrian, R. (2002). Predator functional responses: discriminating between handling and digesting prey. Ecological Monographs, 72(1):95-112. 
Jeschke, J. M., Kopp, M., and Tollrian, R. (2004). Consumer-food systems: why type I functional responses are exclusive to filter feeders. Biological Reviews of the Cambridge Philosophical Society, 79(2):337-349.

Jeschke, J. M. and Tollrian, R. (2005). Predicting herbivore feeding times. Ethology, 111(2):187-206.

Juliano, S. A. (2001). Non-linear curve fitting: Predation and functional response curves. In Scheiner, S. M. and Gurevitch, J., editors, Design and Analysis of Ecological Experiments, pages 178-196. Oxford Univ. Press, New York, 2nd edition.

Kaiser, H. (1983). Small scale spatial heterogeneity influences predation success in an unexpected way: Model experiments on the functional response of predatory mites (Acarina). Oecologia, 56(2-3):249-256.

Kalinkat, G., Brose, U., and Rall, B. C. (2013a). Habitat structure alters top-down control in litter communities. Oecologia, 172(3):877-887.

Kalinkat, G., Jochum, M., Brose, U., and Dell, A. I. (2015). Body size and the behavioral ecology of insects: linking individuals to ecological communities. Current Opinion in Insect Science, 9:24-30.

Kalinkat, G. and Rall, B. C. (2015). Effects of climate change on the interactions between insect pests and their natural enemies. In Björkman, C. and Niemelä, P., editors, Climate Change and Insect Pests, pages 74-91. CABI, Wallingford, UK.

Kalinkat, G., Schneider, F. D., Digel, C., Guill, C., Rall, B. C., and Brose, U. (2013b). Body masses, functional responses and predator-prey stability. Ecology Letters, 16(9):1126-1134.

Karasov, W. H. and McWilliams, S. R. (2005). Digestive constraints in mammalian and avian ecology. In Stark, J. M. and Wang, T., editors, Physiological and ecological adaptations to feeding in vertebrates, pages 88-112. Science Publishers, Enfield, New Hampshire.

Kareiva, P. M., Kingsolver, J. G., and Huey, R. B., editors (1992). Biotic Interactions and Global Change. Sinauer Associates Inc, Sunderland, Mass., 1st edition.

Kéfi, S., Berlow, E. L., Wieters, E. A., Navarrete, S. A., Petchey, O. L., Wood, S. A., Boit, A., Joppa, L. N., Lafferty, K. D., Williams, R. J., Martinez, N. D., Menge, B. A., Blanchette, C. A., Iles, A. C., and Brose, U. (2012). More than a meal. . integrating non-feeding interactions into food webs. Ecology Letters, 15(4):291-300.

Kleiber, M. (1961). The fire of life: an introduction to animal energetics. Wiley, New York. 
Li, Y., Brose, U., Meyer, K., and Rall, B. C. (2017a). How patch size and refuge availability change interaction strength and population dynamics: a combined individual- and population-based modeling experiment. PeerJ, 5:e2993.

Li, Y., Kalinkat, G., and Rall, B. C. (2017b). Joint effects of habitat loss and warming alter species interactions and enhance biodiversity loss. unpublished thesis chapter.

Lotka, A. J. (1925). Elements of Physical Biology. Williams and Wilkins Company, Baltimore.

MacArthur, R. (1955). Fluctuations of animal populations and a measure of community stability. Ecology, 36(3):533-536.

Mantyka-Pringle, C. S., Martin, T. G., and Rhodes, J. R. (2012). Interactions between climate and habitat loss effects on biodiversity: a systematic review and metaanalysis. Global Change Biology, 18(4):1239-1252.

Maselou, D., Perdikis, D., and Fantinou, A. (2015). Effect of hunger level on prey consumption and functional response of the predator Macrolophus pygmaeus. Bulletin of Insectology, 68(2):211-218.

May, R. M. (1972). Will a large complex system be stable? Nature, 238(5364):413414.

McCann, K. S. (2000). The diversity-stability debate. Nature, 405(6783):228-233.

McCann, K. S., Rasmussen, J. B., and Umbanhowar, J. (2005). The dynamics of spatially coupled food webs: Spatially coupled food webs. Ecology Letters, $8(5): 513-523$.

McGill, B. J. and Mittelbach, G. G. (2006). An allometric vision and motion model to predict prey encounter rates. Evolutionary Ecology Research, 8(4):691-701.

Meehan, T. D. (2006). Energy use and animal abundance in litter and soil communities. Ecology, 87(7):1650-1658.

Miller, J. R. B., Ament, J. M., and Schmitz, O. J. (2014). Fear on the move: predator hunting mode predicts variation in prey mortality and plasticity in prey spatial response. Journal of Animal Ecology, 83(1):214-222.

Mols, C. M. M., Oers, K. v., Witjes, L. M. A., Lessells, C. M., Drent, P. J., and Visser, M. E. (2004). Central assumptions of predator-prey models fail in a semi-natural experimental system. Proceedings of the Royal Society of London B: Biological Sciences, 271(Suppl 3):S85-S87.

Munyaneza, J. and Obrycki, J. J. (1997). Functional response of Coleomegilla maculata (Coleoptera: Coccinellidae) to colorado potato beetle eggs (Coleoptera: Chrysomelidae). Biological Control, 8(3):215-224. 
Murdoch, W. W. and Oaten, A. (1975). Predation and population stability. In MacFadyen, A., editor, Advances in Ecological Research, volume 9, pages 1-131. Academic Press, London.

Nunney, L. (1980a). The influence of the type 3 (sigmoid) functional response upon the stability of predator-prey difference models. Theoretical Population Biology, 18(2):257-278.

Nunney, L. (1980b). The stability of complex model ecosystems. The American Naturalist, 115(5):639-649.

Oaten, A. and Murdoch, W. W. (1975a). Functional response and stability in predator-prey systems. The American Naturalist, 109(967):289-298.

Oaten, A. and Murdoch, W. W. (1975b). Switching, functional response, and stability in predator-prey systems. The American Naturalist, 109(967):299-318.

Oksanen, L., Fretwell, S. D., Arruda, J., and Niemela, P. (1981). Exploitation ecosystems in gradients of primary productivity. The American Naturalist, 118(2):240261.

Okuyama, T. (2012). Flexible components of functional responses. The Journal of Animal Ecology, 81(1):185-189.

Ott, D., Digel, C., Rall, B. C., Maraun, M., Scheu, S., and Brose, U. (2014). Unifying elemental stoichiometry and metabolic theory in predicting species abundances. Ecology Letters, 17(10):1247-1256.

Otto, S. B., Rall, B. C., and Brose, U. (2007). Allometric degree distributions facilitate food-web stability. Nature, 450(7173):1226-1229.

Pawar, S., Dell, A. I., and Savage, V. M. (2012). Dimensionality of consumer search space drives trophic interaction strengths. Nature, 486:485-489.

Persson, A. and Brönmark, C. (2002a). Foraging capacities and effects of competitive release on ontogenetic diet shift in bream, Abramis brama. Oikos, 97(2):271-281.

Persson, A. and Brönmark, C. (2002b). Foraging capacity and resource synchronization in an ontogenetic diet switcher, Pikeperch (Stizostedion lucioperca). Ecology, 83(11):3014-3022.

Persson, L., Leonardsson, K., de Roos, A. M., Gyllenberg, M., and Christensen, B. (1998). Ontogenetic scaling of foraging rates and the dynamics of a size-structured consumer-resource model. Theoretical Population Biology, 54(3):270-293.

Petchey, O. L., McPhearson, P. T., Casey, T. M., and Morin, P. J. (1999). Environmental warming alters food-web structure and ecosystem function. Nature, 402(6757):6972 . 
Peters, R. H. (1983). The ecological implications of body size. Number 2 in Cambridge studies in ecology. Cambridge University Press, New York, repr edition.

Pinheiro, J., Bates, D., DebRoy, S., Sarkar, D., and R Core Team (2016). nlme: Linear and Nonlinear Mixed Effects Models. R package version 3.1-128.

Post, D. M., Pace, M. L., and Hairston, N. G. (2000). Ecosystem size determines food-chain length in lakes. Nature, 405(6790):1047-1049.

R Core Team (2016). R: A Language and Environment for Statistical Computing. R Foundation for Statistical Computing, Vienna, Austria.

Rall, B. C., Brose, U., Hartvig, M., Kalinkat, G., Schwarzmuller, F., Vucic-Pestic, O., and Petchey, O. L. (2012). Universal temperature and body-mass scaling of feeding rates. Philosophical Transactions of the Royal Society B: Biological Sciences, 367(1605):2923-2934.

Rall, B. C., Guill, C., and Brose, U. (2008). Food-web connectance and predator interference dampen the paradox of enrichment. Oikos, 117(2):202-213.

Rall, B. C., Kalinkat, G., Ott, D., Vucic-Pestic, O., and Brose, U. (2011). Taxonomic versus allometric constraints on non-linear interaction strengths. Oikos, 120(4):483-492.

Rall, B. C., Vucic-Pestic, O., Ehnes, R. B., Emmerson, M., and Brose, U. (2010). Temperature, predator-prey interaction strength and population stability. Global Change Biology, 16(8):2145-2157.

Real, L. A. (1977). The kinetics of functional response. The American Naturalist, 111(978):289-300.

Rip, J. M. K. and McCann, K. S. (2011). Cross-ecosystem differences in stability and the principle of energy flux: Cross-ecosystem differences in stability. Ecology Letters, 14(8):733-740.

Rosenzweig, M. L. (1971). Paradox of enrichment: destabilization of exploitation ecosystems in ecological time. Science, 171(3969):385-387.

Rosenzweig, M. L. and MacArthur, R. H. (1963). Graphical representation and stability conditions of predator-prey interactions. The American Naturalist, 97(895):209223.

Sala, O. E., Chapin, F. S., Iii, Armesto, J. J., Berlow, E., Bloomfield, J., Dirzo, R., Huber-Sanwald, E., Huenneke, L. F., Jackson, R. B., Kinzig, A., Leemans, R., Lodge, D. M., Mooney, H. A., Oesterheld, M., Poff, N. L., Sykes, M. T., Walker, B. H., Walker, M., and Wall, D. H. (2000). Global biodiversity scenarios for the year 2100. Science, 287(5459):1770-1774. 
Sarnelle, O. and Wilson, A. E. (2008). Type III functional response in Daphnia. Ecology, 89(6):1723-1732.

Savage, V. M., Gilloly, J. F., Brown, J. H., and Charnov, E. L. (2004a). Effects of body size and temperature on population growth. The American Naturalist, 163(3):429-441.

Savage, V. M., Gillooly, J. F., Woodruff, W. H., West, G. B., Allen, A. P., Enquist, B. J., and Brown, J. H. (2004b). The predominance of quarter-power scaling in biology. Functional Ecology, 18(2):257-282.

Scheffer, M. and De Boer, R. J. (1995). Implications of spatial heterogeneity for the paradox of enrichment. Ecology, 76(7):2270-2277.

Schmitz, O. J., Krivan, V., and Ovadia, O. (2004). Trophic cascades: the primacy of trait-mediated indirect interactions. Ecology Letters, 7(2):153-163.

Schmitz, O. J., Post, E., Burns, C. E., and Johnston, K. M. (2003). Ecosystem responses to global climate change: Moving beyond color mapping. BioScience, 53(12):1199-1205.

Schneider, F. D., Brose, U., Rall, B. C., and Guill, C. (2016). Animal diversity and ecosystem functioning in dynamic food webs. Nature Communications, 7:20411723.

Schneider, F. D., Scheu, S., and Brose, U. (2012). Body mass constraints on feeding rates determine the consequences of predator loss. Ecology Letters, 15(5):436-443.

Schröder, A., Kalinkat, G., and Arlinghaus, R. (2016). Individual variation in functional response parameters is explained by body size but not by behavioural types in a poeciliid fish. Oecologia, 182(4):1129-1140.

Scotti, M., Hartvig, M., Winemiller, K., Li, Y., Jauker, F., Jordán, F., and Dormann, C. (in press 2017). Trait-based and process-oriented modeling in ecological network dynamics. In Moore, J., deRuiter, P., Wolters, V., and McCann, K., editors, Food Webs: Science for Impact (in press). Cambridge University Press, Cambridge, UK.

Sentis, A., Hemptinne, J.-L., and Brodeur, J. (2013). Parsing handling time into its components: implications for responses to a temperature gradient. Ecology, 94(8):1675-1680.

Seto, K. C., Dhakal, S., Bigio, A., Blanco, H., Delgado, G. C., Dewar, D., Huang, L., Inaba, A., Kansal, A., Lwasa, S., McMahon, J. E., Müller, D., Murakami, J., Nagendra, H., and Ramaswami, A. (2014). Human settlements, infrastructure and spatial planning. In Edenhofer, O., Pichs-Madruga, R., Sokona, Y., Farahani, E., Kadner, S., Seyboth, K., Adler, A., Baum, I., Brunner, S., Eickemeier, P., Kriemann, 
B., Savolainen, J., Schlömer, S., von Stechow, C., Zwickel, T., and Minx, J. C., editors, Climate Change 2014: Mitigation of Climate Change. Contribution of Working Group III to the Fifth Assessment Report of the Intergovernmental Panel on Climate Change, pages 923 - 1000. Cambridge University Press, Cambridge, United Kingdom and New York, NY, USA.

Shurin, J. B., Clasen, J. L., Greig, H. S., Kratina, P., and Thompson, P. L. (2012). Warming shifts top-down and bottom-up control of pond food web structure and function. Philosophical Transactions of the Royal Society of London B: Biological Sciences, 367(1605):3008-3017.

Smith, P., Bustamante, M., Ahammad, H., Clark, H., Dong, H., Elsiddig, E. A., Haberl, H., Harper, R., House, J., Jafari, M., Masera, O., Mbow, C., Ravindranath, N. H., Rice, C. W., Robledo Abad, C., Romanovskaya, A., Sperling, F., and Tubiello, F. (2014). Agriculture, Forestry and Other Land Use (AFOLU). In Climate Change 2014: Mitigation of Climate Change. Contribution of Working Group III to the Fifth Assessment Report of the Intergovernmental Panel on Climate Change [Edenhofer, O., R. Pichs-Madruga, Y. Sokona, E. Farahani, S. Kadner, K. Seyboth, A. Adler, I. Baum, S. Brunner, P. Eickemeier, B. Kriemann, J. Savolainen, S. Schlömer, C. von Stechow, T. Zwickel and J.C. Minx (eds.)]. Cambridge University Press, Cambridge, United Kingdom and New York, NY, USA.

Smout, S. and Lindstrøm, U. (2007). Multispecies functional response of the minke whale Balaenoptera acutorostrata based on small-scale foraging studies. Marine Ecolog-Progress Series - MAR ECOL-PROGR SER, 341:277-291.

Solomon, M. E. (1949). The natural control of animal populations. Journal of Animal Ecology, 18:1-35.

Spitze, K. (1985). Functional response of an ambush predator: Chaoborus americanus predation on Daphnia pulex. Ecology, 66(3):938-949.

Thompson, R. M., Brose, U., Dunne, J. A., Hall, R. O., Hladyz, S., Kitching, R. L., Martinez, N. D., Rantala, H., Romanuk, T. N., Stouffer, D. B., and Tylianakis, J. M. (2012). Food webs: reconciling the structure and function of biodiversity. Trends in Ecology \& Evolution, 27(12):689-697.

Tylianakis, J. M., Didham, R. K., Bascompte, J., and Wardle, D. A. (2008). Global change and species interactions in terrestrial ecosystems. Ecology Letters, 11(12):1351-1363.

Valiente-Banuet, A., Aizen, M. A., Alcántara, J. M., Arroyo, J., Cocucci, A., Galetti, M., García, M. B., García, D., Gómez, J. M., Jordano, P., Medel, R., Navarro, L., Obeso, J. R., Oviedo, R., Ramírez, N., Rey, P. J., Traveset, A., Verdú, M., and Zamora, R. (2015). Beyond species loss: the extinction of ecological interactions in a changing world. Functional Ecology, 29(3):299-307. 
Van Gils, J. A., Dekinga, A., Spaans, B., Vahl, W. K., and Piersma, T. (2005). Digestive bottleneck affects foraging decisions in red knots Calidris canutus. II. Patch choice and length of working day. Journal of Animal Ecology, 74(1):120-130.

Vasseur, D. A. and McCann, K. S. (2005). A mechanistic approach for modeling temperature-dependent consumer-resource dynamics. The American Naturalist, 166(2):184-198.

Verhulst, P. (1838). Notice sur la loi que la population suit dans son accroissement. Correspondance mathëmatique et physique, 10:113-121.

Vinebrooke, R. D., Cottingham, K. L., Norberg, J., Scheffer, M., Dodson, S. I., Maberly, S. C., and Sommer, U. (2004). Impacts of multiple stressors on biodiversity and ecosystem functioning: the role of species co-tolerance. Oikos, 104(3):451-457.

Volterra, V. (1926). Fluctuations in the abundance of a species considered mathematically. Nature, 118(2972):558-560.

Vucic-Pestic, O., Birkhofer, K., Rall, B. C., Scheu, S., and Brose, U. (2010a). Habitat structure and prey aggregation determine the functional response in a soil predator-prey interaction. Pedobiologia, 53(5):307-312.

Vucic-Pestic, O., Ehnes, R. B., Rall, B. C., and Brose, U. (2011). Warming up the system: higher predator feeding rates but lower energetic efficiencies. Global Change Biology, 17(3):1301-1310.

Vucic-Pestic, O., Rall, B. C., Kalinkat, G., and Brose, U. (2010b). Allometric functional response model: body masses constrain interaction strengths. Journal of Animal Ecology, 79(1):249-256.

Wahlström, E., Persson, L., Diehl, S., and Byström, P. (2000). Size-dependent foraging efficiency, cannibalism and zooplankton community structure. Oecologia, 123(1):138-148.

Wickham, S. A. (1995). Cyclops predation on ciliates: species-specific differences and functional responses. Journal of Plankton Research, 17(8):1633-1646.

Williams, R. J. and Martinez, N. D. (2004a). Limits to trophic levels and omnivory in complex food webs: theory and data. The American Naturalist, 163(3):458-468.

Williams, R. J. and Martinez, N. D. (2004b). Stabilization of chaotic and nonpermanent food-web dynamics. The European Physical Journal B - Condensed Matter, 38(2):297-303.

Yodzis, P. (1981). The stability of real ecosystems. Nature, 289(5799):674-676.

Yodzis, P. and Innes, S. (1992). Body size and consumer-resource dynamics. The American Naturalist, 139(6):1151-1175. 
Zimmermann, B., Sand, H., Wabakken, P., Liberg, O., and Andreassen, H. P. (2015). Predator-dependent functional response in wolves: from food limitation to surplus killing. Journal of Animal Ecology, 84(1):102-112.

Zuur, A. F., Ieno, E. N., and Elphick, C. S. (2010). A protocol for data exploration to avoid common statistical problems: Data exploration. Methods in Ecology and Evolution, 1(1):3-14.

Zuur, A. F., Ieno, E. N., and Smith, G. M. (2007). Analysing Ecological Data. Statistics for Biology and Health. Springer New York, New York, NY.

Zuur, A. F., Ieno, E. N., Walker, N., Saveliev, A. A., and Smith, G. M. (2009). Mixed effects models and extensions in ecology with R. Statistics for Biology and Health. Springer New York, New York, NY. 


\section{Supporting information for Chapter 2}

\section{VIF (variance inflation factor) test on independent variables}

According to Zuur et al. (2010), the independent variables of a linear mixed-effects model shall all have the values of VIF less than 3 to make sure that there are not correlation between them (i.e. multicollinearity). The variance inflation factor test is operated in R (R Core Team, 2016) using the ,corvif' function provided by Zuur et al. (2009). Before the test, we converted the values of binary variable predator satiation level to either zero or one to make this variable valid for such test. All independent variables involved in the analyses of functional response parameters turned out to have VIF values less than 3: predator mass with VIF of 1.64, temperature 1.25, experimental duration 1.33 , predator satiation 2.19 and dimensionality 2.18 .

\section{Partial residual plots}

The plotted points in Fig. 2.3 are presented as $\epsilon_{d / s}+b_{d / s} x_{d / s}+a_{d / s}$ versus $x_{d / s}$, where $\epsilon_{d / s}$ is the residuals of the statistical model for the specific dimensionality or predator satiation, $b_{d / s} x_{d / s}$ represents the fitted coefficient and the plotted independent variable and $a_{d / s}$ is the fitted intercept of the model for that dimensionality or predator satiation. 



\section{Supporting information for Chapter 3}

\section{ODD protocol for in silico feeding experiment}

\section{Overview}

We modeled allometric predator-prey feeding interactions using an individual-based approach. The model description followed the ODD (Overview, Design concepts, Details) protocol (Grimm et al., 2006, 2010). The model was implemented in C++ using Code::Blocks as development environment.

\section{Purpose}

The purpose of the model is to estimate the dependencies of functional-response parameters on patch size and habitat complexity (represented by refuge availability) in a system with one predator and several prey items. As a preparation, we first investigated the maximum feeding rate without any explicit space properties, as we assumed that the maximum feeding rate is driven by physiological (mechanical) parameters such as chewing and digesting and does not scale with patch size or habitat complexity. Secondly, we assessed functional responses of predators to different prey densities in patches of different sizes and refuge availability.

\section{Entities, state variables and scales}

One predator and several prey individuals make up the agents of the model. The common state variables of predator and prey are individual identity, spatial coordinates (in $\mathrm{cm}$ ), body mass (in $\mathrm{mg}$ ), and body-mass dependent velocity. The predator is characterized by further state variables related to hunting and digestion. The parameter ,gut-fill' captures how many milligrams of food are currently in the gut of the predator, ,still-handling' captures how many time steps are still needed for handling prey, and ,prey-eaten' counts the number of prey items consumed by the predator.

The environment consists of a two-dimensional square area where its size and habitat complexity can be modified. To manipulate habitat complexity in the form of refuge availability for the prey, each cell in this area is characterized by the boolean state 
variable ,prey hiding'. This variable is set to 1 if prey individuals staying in this cell cannot be found by a predator, and to 0 otherwise. The predator and prey can move continuously in the area via random walk. Predator and prey are both able to enter all the cells, but the predator is not able to find prey in refuge cells. We implemented non-periodic wall-like boundary conditions (Attard, 2006) to simulate a finite-sized patch.

In terms of scales, one grid cell is always $1 \mathrm{~cm} \times 1 \mathrm{~cm}$ and the spatial extent of the patch is modified from $20 \mathrm{~cm} \times 20 \mathrm{~cm}$ to $1000 \mathrm{~cm} \times 1000 \mathrm{~cm}$ to account for different patch sizes. One time step represents one second and the simulations are ran for 3600 time steps corresponding to one hour.

\section{Process overview and scheduling}

The first process that is applied in this discrete-time model is prey movement (random walk with randomly chosen direction and allometric distance, i.e. the velocity of an individual scales with its body mass). The following processes are the predator's decisions and actions (Fig. 3.1 in the main text). First, the predator digests and afterwards if it has caught prey previously and is still handling it, the predator does the process of handling. Subsequently, if the predator is not handling prey anymore and its gut is full (gut-fill $>=60 \%$ ), it rests, i.e. it does not take any actions in this time step. If the predator is not handling prey and is hungry (gut-fill' $<60 \%$ ), the predator moves according to the same rules as prey. After reaching the new position, the predator investigates if it encounters a prey in the current cell. If there is a prey individual in the same cell and this cell is not marked as refuge, the prey will be attacked. If the attack is successful, another prey item is placed randomly into the grid to keep prey density constant. The predator starts to handle the prey in the next time step.

\section{Design concepts}

Basic principles - This model mimics classical functional response experiments in the laboratory, but the model allows to explore much larger patch sizes than in a real laboratory arena. Moreover, the model can more easily be used to determine maximum feeding rates than laboratory experiments, because the amount of prey can be held constantly until maximum feeding rates are reached. Emergence Functional responses are the main emerging pattern from the model, arising from the predator's efficiency at catching prey in patches of different sizes and habitat complexity (refuge availability). Sensing - Predator and prey are able to detect each other when they meet in the same cell which is not marked as refuge. Both predator and prey are able to detect patch edges and stop nearby when they reach an patch edge. Next time when this individual needs to move, it just moves according to the same rules as before. Interaction - The predator interact with prey by feeding on the prey when they meet on the same cell and the prey is not hidden. When the prey 
is in a refuge cell, the predator can enter that cell, but does not interact with the prey. Stochasticity - Random numbers are used in initialization of most variables, including coordinates of agents, the refuge availability of cells and the state variable ,gut-fill' of the predator. Stochasticity is also involved in the moving direction of agents when random walk applies. Bernoulli-distributed random numbers are drawn to determine the handling time for a prey item as time is a discrete variable in this model. Observation - In each in silico experiment, the number of prey items eaten by the predator is recorded at each time step. At the end of each simulation run, patch size, refuge availability (percentage of refuge cells), initial prey number, and ,prey-eaten' are recorded.

\section{Initialization}

Random values are used to initialize the spatial coordinates of all agents and choose refuge cells. All other initial parameters are listed in Table S3.1.

Tab. S3.1: Agents' state variables and parameters

\begin{tabular}{llll}
\hline sort & variables & unit & note \\
\hline \multirow{3}{*}{ state variable } & gut-fill & rate & randomly initialized \\
& still-handling & $\mathrm{s}$ & initially set to zero \\
& individual identify & number & \\
& prey-eaten & number & initially set to zero \\
\hline \multirow{3}{*}{ calculated parameter } & velocity & $\mathrm{cm} \mathrm{s}^{-1}$ & \\
& rate of successful attack & rate & \\
& full gut & $\mathrm{mg}$ & allometrically calculated \\
& digestion rate & rate & \\
& handling time & $\mathrm{s}$ & \\
\hline
\end{tabular}

Most species traits regulating the processes described above are calculated by allometric rules. These include velocities of predator and prey $V\left[\mathrm{~cm} \mathrm{~s}^{-1}\right]$, Eq. (S3.1a), (Peters, 1983), and the predator-specific traits: gut size $G$ [mg ], Eq. (S3.1b), (Ibarrola et al., 2012); digestion rate $D\left[\mathrm{mg} \mathrm{s}^{-1}\right]$, Eq. (S3.1c), (Ibarrola et al., 2012); handling time $T_{h}$ [s], Eq. (S3.1d), (modified from Rall et al., 2012); and rate of successful attack $S_{a}$ unitless, Eq. (S3.1e), (Wahlström et al., 2000; data from Gergs 
and Ratte, 2009, and Gergs, 2011):

$$
\begin{aligned}
V & =v_{0} M^{a_{v}} \\
G & =g_{0} M^{a_{g}} \\
D & =d_{0} M^{a_{d}} \\
T_{h} & =h_{0} M_{p}^{a_{h, p}} M_{n}^{a_{h, n}} \\
S_{a} & =a_{0}\left(\frac{R}{R_{o p t}} e^{1-\frac{R}{R_{o p t}}}\right)^{\lambda}
\end{aligned}
$$

where $v_{0}, g_{0}$ and $d_{0}$ are constants, $a_{v}, a_{g}$, and $a_{d}$ are the allometric exponents, and $M$ is the body mass of the corresponding individual. Subscripts ${ }_{p}$ and ${ }_{n}$ of $M$ indicate predator and prey, respectively. As only few relevant studies were found on digestion mechanisms, we used generalized Ricker's function (Persson et al., 1998; Persson and Brönmark, 2002b,a; Wahlström et al., 2000; Brose et al., 2008; Rall et al., 2011) to describe the scaling of attack success $\left(S_{a}\right)$ depending on body size. This function consists of the maximum attack success $a_{0}$, predator-prey body-mass ratio, $R$ and its optimum $R_{\text {opt }}$ and a shaping parameter, $\lambda$. Predator and prey also possess some state variables to assist their decision making and activities, i.e. ,position' for all individuals; ,gut fullness' and ,still handling' for the predator; ,prey identity' for prey.

\section{Submodels}

1. Prey move. This process is the first one for each time step. Prey individuals do random walks consecutively according to their identity number. A random direction is generated (a double precision floating number from 0 to $2 \pi$ ) and position changes vertically and horizontally are calculated according to prey velocity. Before updating the actual coordinates, wall boundary conditions are considered, checking if values of the coordinates would be beyond the boundaries. If so, the value is set close to the coordinate value of that edge but with a distance to the edge of $10^{-6} \mathrm{~cm}$.

2. Digest. The state variable ,gut-fill' of the predator is subtracted by ,digestion rate' in this process. It is executed each time step even when the gut is already empty. If the value of gut fill drops below zero, it is set to zero.

3. Handle prey. This process is executed under the condition that the state variable ,still-handling' has a positive value. The value of ,still-handling' is reduced by one in this process.

4. Move. The predator does a random walk. If the predator is satisfied, meaning ,gut-fill' exceeds 0.6 , this process is skipped.

5. Encounter and attack? Here, the coordinate of the predator is checked only when it is about to hunt (gut-fill ${ }^{\star}<0.6$ ). If the predator is currently in a refuge cell, the hunting process is forfeited. If it is not forfeited, the following actions are 
executed. 1) Check potential prey, checking if there exists one prey item that is in the same cell as the predator. Checking order follows the prey's identity numbers. As soon as one prey fulfills the condition, the checking is finished. 2) If there is a potential prey item, a random number (ranging from 0 to 1 ) is generated and compared to ,rate of successful attack' to decide if this prey flees.

6. Attack success? If the attacked prey does not flee (attack success), values of ,gut-fill' and ,still-handling' will be increased by the amounts calculated from the prey mass and ,prey-eaten' will be increased by one. As time is discrete in our model, a Bernoulli-distributed random number is drawn to make sure that the value of ,still-handling' is an integer and on average still satisfying the calculated handling time. If the prey flees (attack unsuccessful), this time step ends.

7. Replace caught prey. If attack succeeds, the killed prey item (i.e. its identity number) would be randomly given a new set of spatial coordinates, but body mass never changes.

8. Output data. Data are recorded immediately after each of 3600 time steps. The number of prey eaten and relevant input values are recorded, i.e. body masses of agents, patch size, percentage of refuge cells and initial number of prey items.

\section{Allometric handling time}

We used data from Rall et al. (2012) to parameterize the equation for handling time $\left(T_{h}\right.$, Eq. (3.2d)). We only selected the data for predation (parasitism excluded) and for short experimental duration ( $\leq 10$ minutes) to make the analysis. We fitted 67 data points to a linear mixed-effects model (,lme' in the package ,nlme' in R, Pinheiro et al. (2016); R Core Team (2016)). To correct for differences between studies, study identity was used as a random factor, and all the variables (explanatory variables, body masses of predator and prey species and dependent variable handling time) were ln-transformed. The statistics showed that the handling time increased with increasing prey mass and decreased with increasing prey mass and decreases with increasing predator mass (Fig. S3.1, Table S3.2).

Tab. S3.2: Statistics for handling time ${ }^{a}$

\begin{tabular}{llll}
\hline & Estimate & S.E. & p-value \\
\hline intercept & 3.624 & 0.839 & 0.0001 \\
ln.pred $^{b}$ & -0.330 & 0.059 & $<0.0001$ \\
ln.prey $^{c}$ & 0.173 & 0.051 & 0.0013 \\
\hline
\end{tabular}

\footnotetext{
${ }^{a}$ handling time is ln-transformed

${ }^{b}$ ln-transformed predator body mass

${ }^{c}$ ln-transformed prey body mass
} 

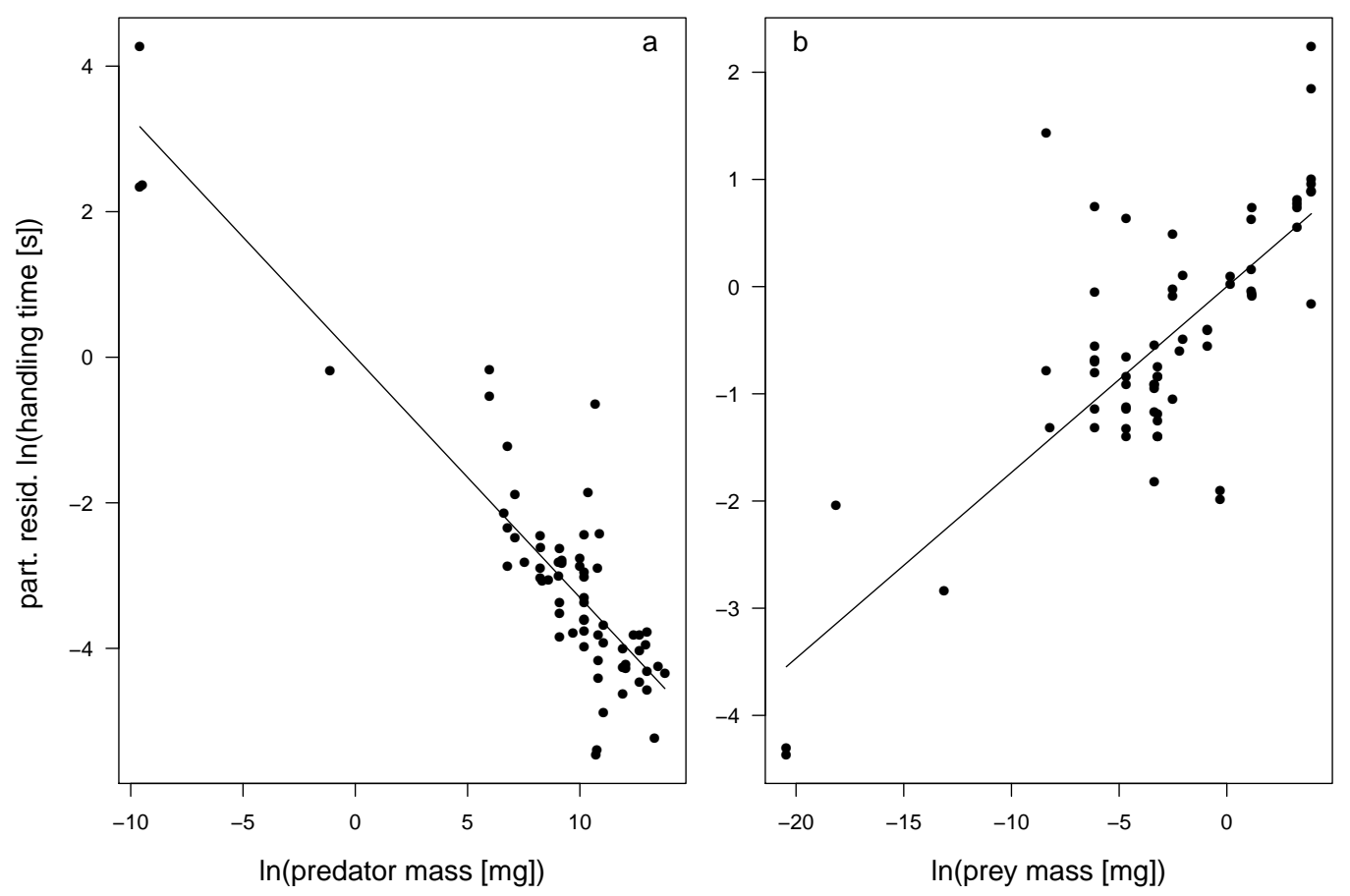

Fig. S3.1: Statistical results for handling time $\left(T_{h}\right)$ show that handling time decreases with increasing predator body mass and increases with increasing prey body mass. Data are ln-transformed before fitting. The partial residual of handling time is used as y-axis.

\section{Preliminary model selection}

We listed the statistical results of ,preliminary tests“ (section „Functional response fitting" in the main text) on the types of scaling that functional-response parameters had with patch size or refuge availability, Tab. S3.3. For the simulations on predatorprey body-mass ratio of 100 and 200, the selected best model (according to the BIC) includes (1) half saturation density scaling with power law to patch size and exponentially to refuge availability; (2) Hill exponent scaling with power law to both patch size and refuge availability. For the simulation on body-mass ratio of 50, (1) the model is selected for the same scaling relationships of half saturation density as for body-mass ratio of 100 and 200; thus, (2) Hill exponent scales exponentially with both patch size and refuge availability.

\section{In silico feeding experiments on other body-mass}

\section{ratios}

We additionally did functional response simulations for predator mass of $200 \mathrm{mg}$ and $50 \mathrm{mg}$ (prey mass of $1 \mathrm{mg}$ ). However, we reduced the numbers of patch sizes and refuge availabilities. The simulated patch sizes for both predator masses are $0.04 \mathrm{~m}^{2}, 0.64 \mathrm{~m}^{2}, 2.56 \mathrm{~m}^{2}, 16 \mathrm{~m}^{2}, 49 \mathrm{~m}^{2}$ and $64 \mathrm{~m}^{2}$; and the simulated refuge 
Tab. S3.3: Full model selection on scaling rules of functional-response parameters

\begin{tabular}{clll}
\hline \multirow{2}{*}{ model scaling rules $^{a b}$} & \multicolumn{3}{c}{ BIC } \\
\cline { 2 - 4 } & 100 & 200 & $50^{c}$ \\
\hline eeee & 45061.65 & 5710.646 & 3930.403 \\
eepe $^{d}$ & 45083.81 & 5709.091 & 3933.047 \\
peee & 45026.84 & 5709.33 & $\underline{3928.032}^{e}$ \\
eeep & 45057.89 & 5706.494 & 3930.711 \\
epee & 45219.88 & 5741.362 & 3940.024 \\
pepe & 45025.2 & 5707.415 & 3930.593 \\
eepp & 45091.41 & 5705.066 & 3932.338 \\
eppe & 45218.91 & 5754.17 & 3944.321 \\
peep & 45025.39 & 5706.341 & 3928.081 \\
ppee & 45228.25 & 5735.171 & 3938.638 \\
epep & 45231.84 & 5734.309 & 3940.953 \\
pepp & 45023.57 & 5703.671 & 3930.069 \\
pppe & 45185.07 & 5733.498 & 3941.549 \\
eppp & 45223.9 & 5731.754 & 3944.204 \\
ppep & 45236.62 & 5737.974 & 3944.041 \\
pppp & 45189.86 & 5729.644 & 3941.185 \\
\hline
\end{tabular}

${ }^{a}$ scaling of $N_{0}$ to $A, N_{0}$ to $R, h$ to $A$ and $h$ to $R$, subsequently

${ }^{b} N_{0}$ half saturation density, $h$ Hill exponent, $A$ patch size, $R$ refuge availability

${ }^{c}$ predator-prey body-mass ratio

${ }^{d} \mathrm{e} / \mathrm{p}$ : exponential/power law scaling

${ }^{e}$ lowest BIC value
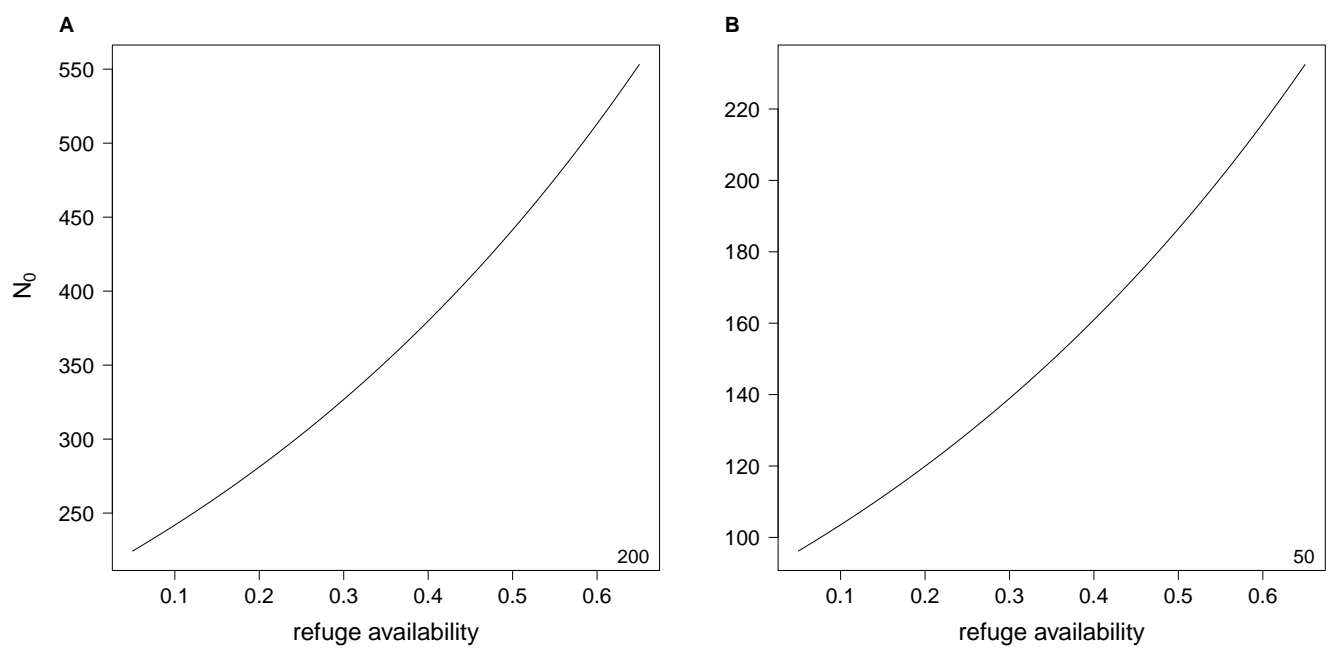

Fig. S3.2: Effect of habitat complexity (expressed as refuge availability) on half saturation density. Panel A and B show the results for body-mass ratio of 200 and 50, respectively (right-bottom corner).

availabilities are $5 \%$ to $65 \%$, in steps of $15 \%$. All the following statistics follow the descriptions in the paper.

The maximum feeding rate, $f_{\max }$, were 4.12 and 10.56 individuals for predator 
Tab. S3.4: Statistical results of functional-response parameters simulated on other bodymass ratios

\begin{tabular}{|c|c|c|c|c|}
\hline predator mass & parameter $^{a}$ & estimate & s.e. & $\mathrm{p}$-value \\
\hline 200 & $\begin{array}{l}f_{\max } \\
b_{N_{0}} \\
\ln \left(C_{N_{0}}\right) \\
\ln \left(C_{h}\right)\end{array}$ & $\begin{array}{l}10.56 \\
1.504 \\
5.338 \\
0.25\end{array}$ & $\begin{array}{l}0.04 \\
0.159 \\
0.067 \\
0.028\end{array}$ & $\begin{array}{l}<2 \times 10^{-16} \\
<2 \times 10^{-16} \\
<2 \times 10^{-16} \\
<2 \times 10^{-16}\end{array}$ \\
\hline 50 & $\begin{array}{l}f_{\max } \\
b_{N_{0}} \\
\ln \left(C_{N_{0}}\right) \\
\ln \left(C_{h}\right)\end{array}$ & $\begin{array}{l}4.12 \\
1.423 \\
4.503 \\
0.26\end{array}$ & $\begin{array}{l}0.07 \\
0.257 \\
0.110 \\
0.047\end{array}$ & $\begin{array}{l}<2 \times 10^{-16} \\
3 \times 10^{-8} \\
<2 \times 10^{-16} \\
3 \times 10^{-8}\end{array}$ \\
\hline
\end{tabular}

${ }^{a}$ refer to Eq. (3.5) in the main text
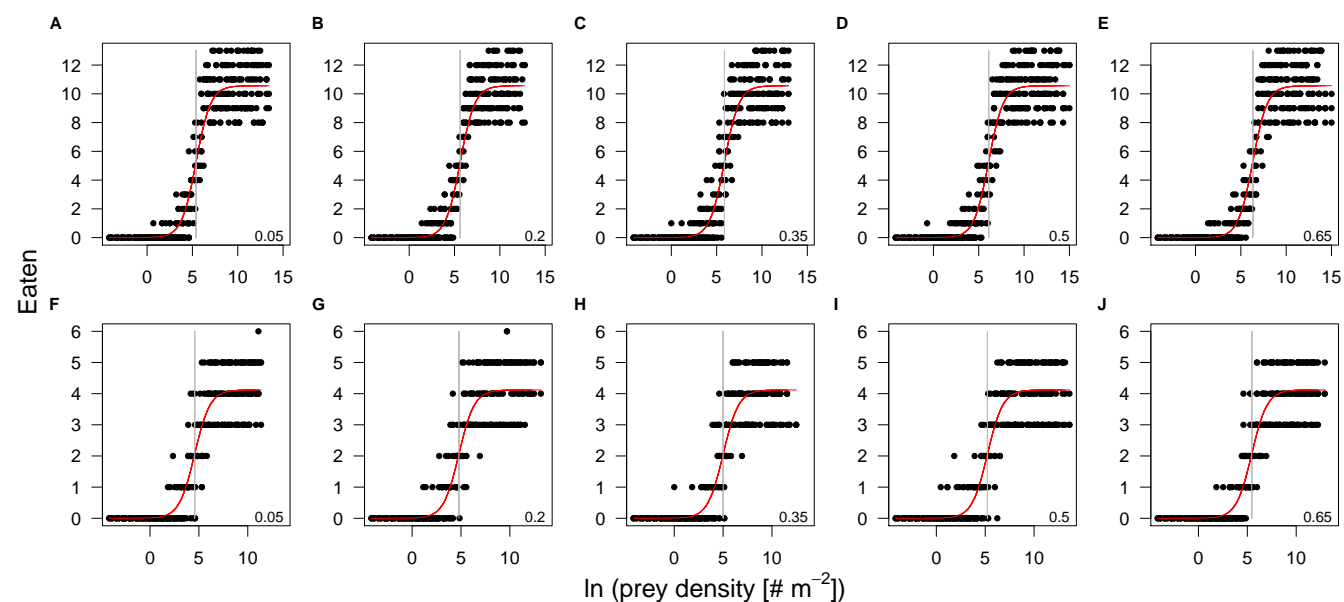

Fig. S3.3: Results of in silico functional response experiments (black dots) and their corresponding fits (red lines). The panels are arranged by increasing refuge availability and increasing body-mass ratios of predator-prey. The upper row (panels A to E) are for body-mass ratio of 200 and lower (panels $F$ to $\mathrm{J}$ ) for 50 . Each row starts with data of $5 \%$ refuge availability (A, uppermost left) to $65 \%$ (O, lowermost right). All prey densities are ln-transformed. The grey vertical lines denote the half saturation densities.

mass of $50 \mathrm{mg}$ and $200 \mathrm{mg}$ (Tab. S3.4). The final models for predator mass of $50 \mathrm{mg}$ and $200 \mathrm{mg}$ based on the BIC selection showed the same dependencies, including an exponential scaling of half saturation density with refuge availability but no scaling with patch size (refer to Eq. (3.5a)). Consistent with the result for predator mass of $100 \mathrm{mg}$ in the main text, they did not include any scaling of Hill exponent for both predator masses (refer to Eq. (3.5b)). The half saturation densities increased with increasing refuge availability, $b_{N_{0}}=1.504$ for predator mass of $200 \mathrm{mg}$ and $b_{N_{0}}=1.423$ for predator mass of $50 \mathrm{mg}$, see Fig. S3.2 and Tab. S3.4. The ln-transformed intercepts of the half saturation density were 5.338 and 4.503 for predator mass of $200 \mathrm{mg}$ and $50 \mathrm{mg}$, respectively (Tab. S3.4). The estimated Hill exponents across all patch sizes and refuge availabilities were 1.284 
for predator mass of $200 \mathrm{mg}$ and 1.300 for predator mass of $50 \mathrm{mg}$ (Tab. S3.4). The fitted functional response curves are of the same shape but feedings are realized at higher densities with increasing refuge availabilities (Fig. S3.3). 



\section{Supporting information for Chapter 4}

\section{Details on meta-data and statistical analyses}

\section{Preprocessing of the meta-data}
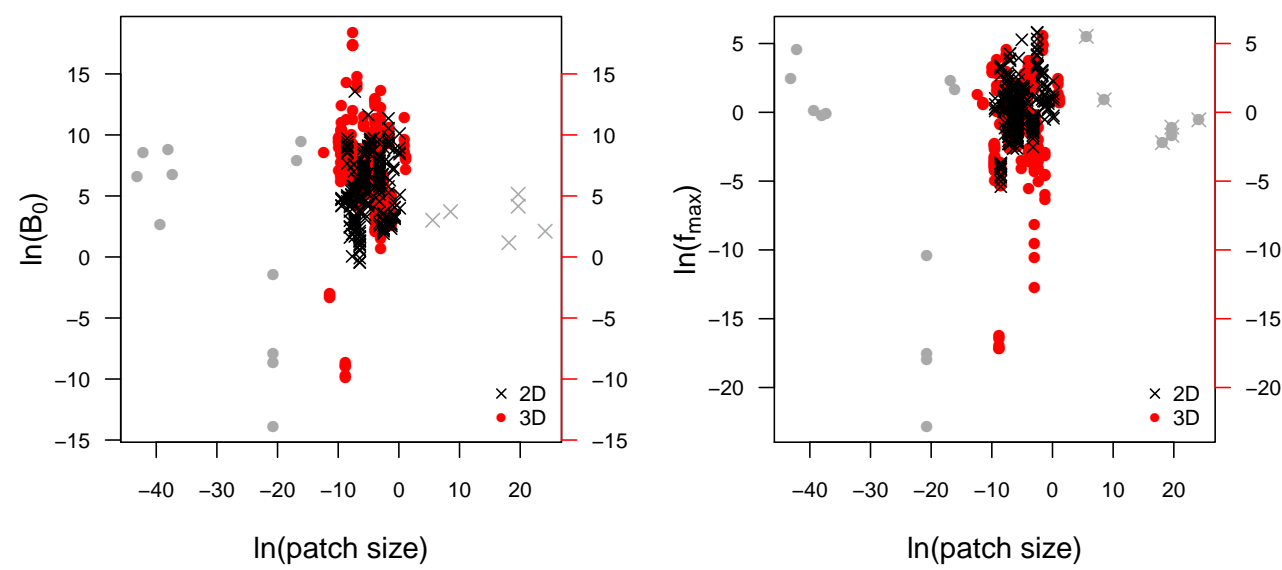

Fig. S4.1: Deleted data from the meta-data. Excluded data points are marked gray which are out of two standard deviations of mean patch sizes $\left(\mathrm{m}^{2} \mid \mathrm{m}^{3}\right)$ in both twoand three-dimensional spaces (see legends). Data plotted are ln-transformed.

We excluded the data points which are out of the two standard deviations of the mean patch sizes before the statistical analysis (see Fig. S4.1). Besides, we also did a test on variance inflation factor (VIF) before data analysis. All the independent variables (predator mass, temperature, patch size and dimensionality) that we included in the statistical models have VIF values less than 3 (Zuur et al., 2010), meaning that there's no evidence for collinearity in these variables. Therefore, it's valid to do the analysis that we carried out for meta-data. 


\section{Selection of random structures}

The table S4.1 illustrates how we selected random structures for the statistical models of functional response parameters, half-saturation density and specific maximum feeding rate.

Tab. S4.1: Selection of random structures used in models analyzing functional response parameters

\begin{tabular}{llll}
\hline FR parameter & fixed structure & random structure & BIC \\
\hline & & $1+\ln \left(M_{c}\right) \mid \mathrm{EM}^{c} / \mathrm{P}^{d}$ & 2152.384 \\
$\ln \left(B_{0}\right)^{a}$ & $\left(\ln \left(M_{c}\right)+\frac{T-T_{0}}{k T T_{0}} * \ln (A)\right) * D^{b}$ & $1 \mid \mathrm{EM} / \mathrm{P}^{e}$ & 2163.007 \\
& & $1+\ln \left(M_{c}\right)^{f} \mid \mathrm{P}$ & $\underline{2129.922}$ \\
& & $1 \mid \mathrm{P}$ & 2153.044 \\
& & $1+\ln \left(M_{c}\right) \mid \mathrm{EM} / \mathrm{P}$ & 2115.123 \\
$\ln \left(f_{\text {max }}\right)^{g}$ & $\left(\ln \left(M_{c}\right)+\frac{T-T_{0}}{k T T_{0}} * \ln (A)\right) * D^{h}$ & $1 \mid \mathrm{EM} / \mathrm{P}$ & 2098.115 \\
& & $1+\ln \left(M_{c}\right) \mid \mathrm{P}$ & 2096.333 \\
& & $1 \mid \mathrm{P}$ & $\underline{2094.934}$ \\
\hline
\end{tabular}

${ }^{a}$ half saturation density

${ }^{b}$ see Eq. (4.3a)

${ }^{c}$ ecosystem type (terrestrial, freshwater or marine) plus metabolic type of the predator (invertebrate, ectothermic vertebrate or unicell)

${ }^{d}$ publication name

${ }^{e}$ the factor $\mathrm{P}$ is nested in the factor EM

${ }^{f}$ random factor affecting both intercept (1) and predator mass $\left(\ln \left(M_{c}\right)\right)$ of the model

${ }^{g}$ specific maximum feeding rate

${ }^{h}$ see Eq. (4.3b)

\section{Partial residuals plots}

The data points plotted in Fig. 4.3 are presented as $\epsilon_{d}+b_{d} x_{d}+a_{d}$ versus $x_{d}$ where $\epsilon_{d}$ is the residuals of the statistical model for the specific dimensionality, $b_{d} x_{d}$ represents the fitted coefficient and the plotted independent variable for that dimension and $a_{d}$ is the fitted intercept of the model for that dimension.

\section{Data points with large deviations}

The line of data points at $\ln$ (patch size) of -3 which lining across a large range of ,partial residual $\ln \left(B_{0}\right)^{\text {‘ }}$ are the data from Galarowicz and Wahl (2005), see Fig. 4.3B. In this study, Galarowicz and Wahl made feeding trails not only with the suitable prey for that stage of the predator but also with the unsuitable prey, resulting the long line at $\ln$ (patch size) of -3 . 


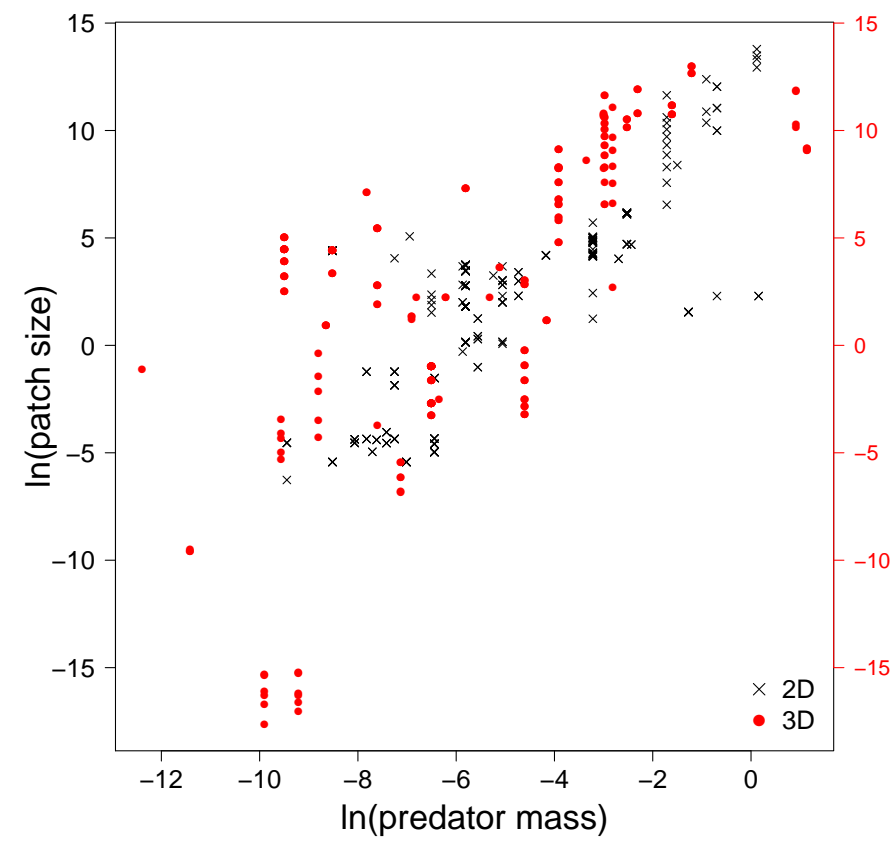

Fig. S4.2: Plot of predator body mass versus patch size. This figure demonstrates the range of patch sizes $\left[\mathrm{m}^{2} \mid \mathrm{m}^{3}\right]$ for all predator body masses [ $\left.\mathrm{mg}\right]$. Data points for twoand three-dimensional spaces are plotted separately (see legend). 


\section{Patch size and predator body mass}

Our meta-data shows that the predator body mass and the size of the patch where the feeding trails are conducted is positively related (Fig. S4.2). Yet for each predator mass, the associated patch sizes still exhibit a considerable variation.

\section{Timeseries of some selected food chain}

\section{simulations}
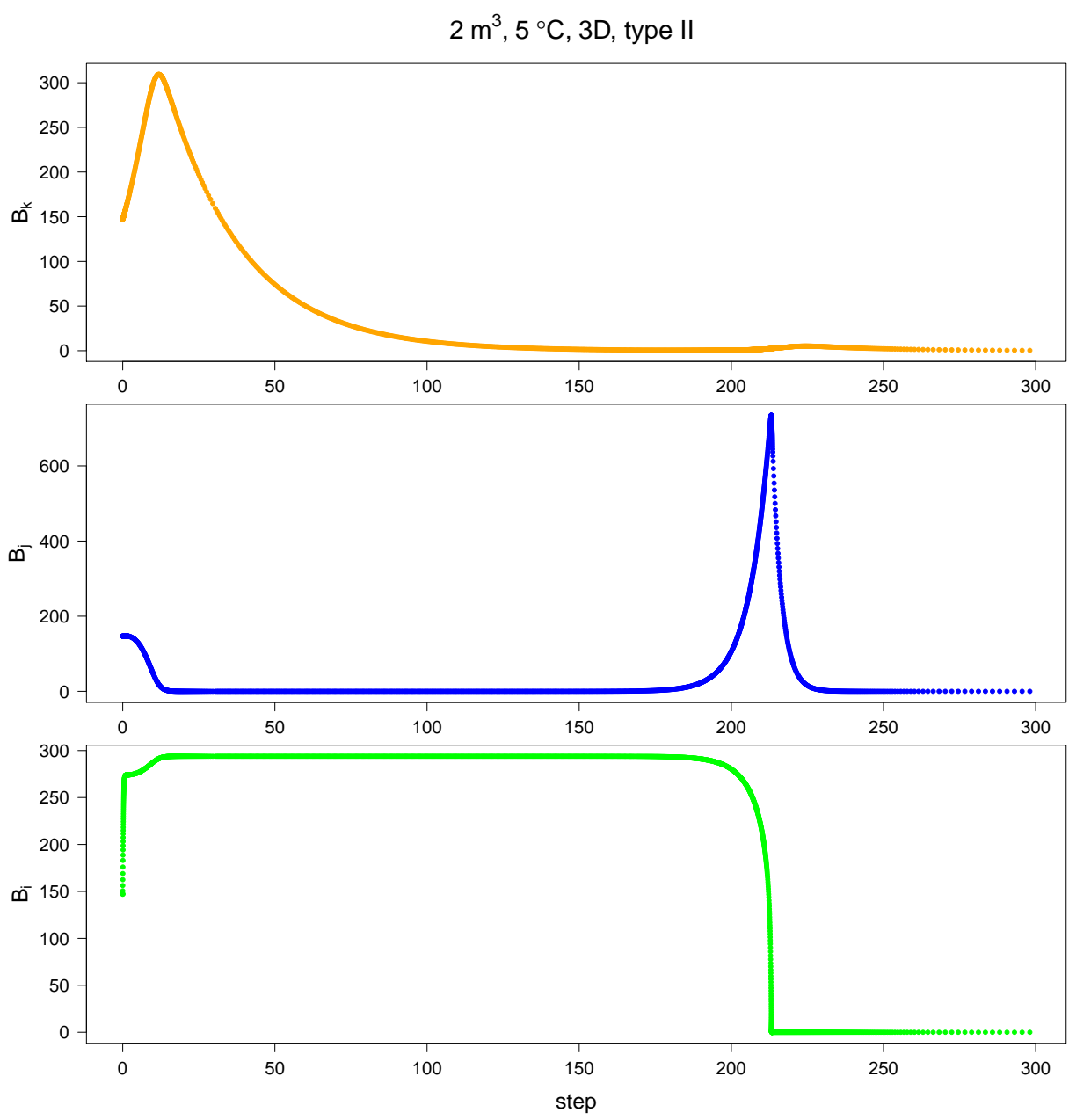

Fig. S4.3: Timeseries of a food chain simulation in three-dimensional space. The simulation shown utilizes the type II functional response and the simulated patch size and temperature are shown. The biomass changes of basal species $\left(B_{i}\right)$, intermediate consumer $\left(B_{j}\right)$ and top predator $\left(B_{k}\right)$ over time (x-axis) are illustrated. The ending biomasses are stable for this simulation. 


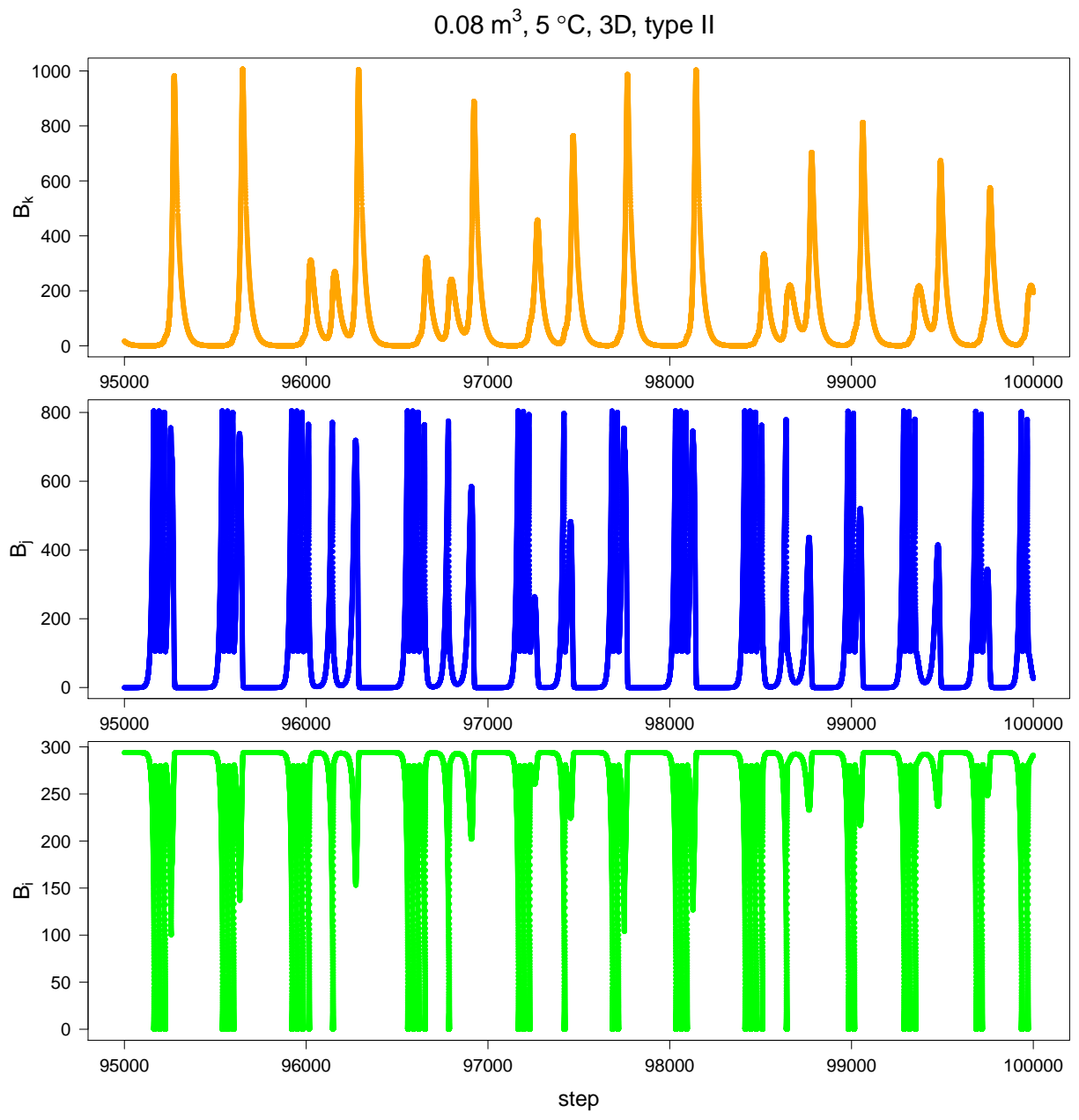

Fig. S4.4: Timeseries of a food chain simulation in three-dimensional space. The simulation shown utilizes the type II functional response and the simulated patch size and temperature are shown. The biomass changes of basal species $\left(B_{i}\right)$, intermediate consumer $\left(B_{j}\right)$ and top predator $\left(B_{k}\right)$ over time (x-axis) are illustrated. The biomasses exhibit cycling dynamics for this simulation. 



\section{Acknowledgement}

I thank Uli for making all of my $\mathrm{PhD}$ life possible. Everything would not have been possible if he decided not to hire me at the first place. He has been kind, encouraging and supporting. His tolerance to the projects that I was interested and conduced allows me to find my own way in theoretical ecology. As I was a total newbie in food web study at the beginning of my $\mathrm{PhD}$, his patience when explaining the basic principles of food web calmed me down and encouraged me to stay in this field. His academic advice has always been enlightening for me.

I would also like to thank my second supervisor, Kerstin Wiegand. Without her agreement to be my supervisor, I would not have been started my official $\mathrm{PhD}$ life. Even though we did not make a project together, it was her who provided me the first step on the individual-based modeling and the world of $\mathrm{C}++$.

I especially thank Björn for all he has done for me. He has done a superior work in supervising my projects, comforting me when I got mad, dealing with my chaotic personality and most importantly cooking nice food with Ellen and me occasionally.

I would like to express my appreciation for Gregor's excellent idea. Without him, two of my research chapters would not have existed. He has also been an extremely good co-author, providing fast and creative collaboration.

I would also like to thank Katrin. She provided a lot of detailed help which enabled me to write code with confidence. I also appreciated for her appearing at the beginning, serving as a role model for my early academic life.

I thank Sebastian Hanß, Konglin Zhu, Xiaowei Wang and Christian for their help of different sorts with respect to modeling. Without these help, I may not be able to comfortably become a theoretical ecologist.

I thank all the members in our research group (FOR 1748), the „Networks On Networks". Through all the formal and informal meetings, I got to know many fields of networking studies and the routines of theoretical work.

I would like to thank Shaopeng and Wanlu. Even though I just got to know them not more than two years, they have become my „mentor parents“. I thank Tingting, „Kalok and Kevin“, Kristy, Ellen and „Chelmuge and Narisu“ for their patience to listen to my blah and providing me nice feeding experiences when necessary. I thank Juan for appearing at the last period of my PhD. Even though she has been (well, 
she will always be) a tiny little person, she provided me with tremendous comforting physically and mentally.

I thank Martin for bringing me into the world of extreme sports, climbing and marathon and his endless patience when I stuck on the wall. I thank Bernhard for his company of actually running the first half marathon. Without these extreme experience and the continuous joy by practicing yoga, I would not have been gone through the mental suffering during my $\mathrm{PhD}$.

I additionally thank Ka, Yanhao and Philipp for their careful and enlightening proofreading. Last but least, I thank for all the other members in my thesis committee. Without your support, I would not be able to have the chance to finish the last step of my PhD! 


\section{Declaration/Eidesstattliche Erklärung}

I, Yuanheng Li, hereby declare that I am the sole author of this thesis. All the references and data sources that were used have been acknowledged. I further declare that this work has never been submitted in any forms as part of any other thesis procedure.

Göttingen, Mar. 03, 2017

Yuanheng Li 



\section{Curriculum Vitae}

\section{Yuanheng Li}

German Centre for Integrative Biodiversity Research (iDiv) Halle-Jena-Leipzig Deutscher Platz 5e, 04103 Leipzig

e-mail: li_yuanheng_eco@126.com

\section{Education}

Since 2015

Since 2013

2010-2013

2005-2009
Continuing as $\mathrm{PhD}$ student,

Theory in Biodiversity Group (Supervisor Ulrich Brose), German Center for Integrative Biodiversity Research (iDiv) Halle-Jena-Leipzig and Friedrich-Schiller-University Jena, Germany

PhD student, Systemic Conservation Biology Group (Supervisor Ulrich Brose),

J.F. Blumenbach Institute of Zoology and Anthropology at the Georg-August-University Göttingen, Germany

M.Sc. in Ecology,

University of Bremen, Germany

B.Sc. in Forestry,

Guizhou University, China

\section{Publications}




\section{Peer-reviewed publications}

Li, Y., Brose, U., Meyer, K. and Rall, B.C. (2017). How patch size and refuge availability change interaction strength and population dynamics: a combined individual- and population-based modeling experiment. PeerJ, 5:e2993.

\section{Book chapters}

Scotti, M., Hartvig, M., Winemiller, K.O., Li, Y., Jauker, F., Jordán, F. and Dormann, C. (2017 (expected)). Trait-based and process-oriented modeling in ecological network dynamics. In Moore, J., deRuiter, P., Wolters, V., and McCann, K., editors, Food Webs: Science for Impact (in press). Cambridge University Press, Cambridge, UK.

\section{Master's thesis}

Li, Y. (2012). Influence of inorganic and organic N sources on two arbuscular mycorrhizal (AM) and one ectomycorrhizal (ECM) tree species: A chamber experiment with tree saplings inoculated with natural mycorrhizal communities. University of Bremen, Germany

\section{Conferences and workshops}

Dec. 2016

Dec. 2015

Sep. 2015

Nov. 2013

Jul. 2013
Li, Y., Kalinkat, G. and Rall, B.C. (talk). Joint effects of habitat loss and warming on trophic interactions and biodiversity. Annual Meeting of British Ecological Society (BES), Liverpool, Britain.

Li, Y., Rall, B.C. and Kalinkat, G. (poster). Effects of space and time on feeding interaction strength. Annual Conference of German Center for Integrative Biodiversity Research (iDiv), Leipzig, Germany.

Li, Y. (talk). Spatial properties affect interaction strength and dynamics of meta-community. 45th Annual Meeting of Gesellschaft für Ökologie (Gfö), University of Göttingen, Germany.

Food Webs: Science for Impact (Decennial Meeting), Giessen, Germany (attended).

An Introduction to Bayesian Modeling for Ecologists (Summer School), Freiburg, Germany (attended). 
